20

\title{
Epitaxial Growth of Thin Films
}

Harald Brune

This chapter gives an introduction to the epitaxial growth of thin films on solid substrates. The term epitaxy refers to the growth of a crystalline layer on (epi) the surface of a crystalline substrate, where the crystallographic orientation of the substrate surface imposes a crystalline order (taxis) onto the thin film. This implies that film elements can be grown, up to a certain thickness, in crystal structures differing from their bulk. If film and substrate have the same crystal lattices, but different lattice constants, the film will be under strain, that is, it will have a slightly different lattice constant than in its own bulk. Both effects, together with the electronic hybridization at the interface, lead to novel properties.

One distinguishes homo- and heteroexpitaxy, where the former refers to the growth on one element on a crystal surface of its own and the latter refers to the more general case, where film and substrate materials are different. Note that the first distinguishes itself from crystal growth, as we will see in more detail later.

We start this chapter by giving examples from technology, illustrating where thin epitaxial films are used and outlining potential applications that become reality once we are able to grow the respective thin film sequences. We then contrast thin film and crystal growth, respectively, with kinetics and thermodynamics of growth. We introduce the deposition techniques used in epitaxial thin film growth and then discuss the classical thermodynamic approach, which led to the definition of the growth modes. These modes refer to the morphology taken on by a system grown close to thermodynamic equilibrium. Often films are grown far away from equilibrium and their morphology is determined by kinetics, that is, it is the result of the microscopic path taken by the system during growth. This path is determined by the hierarchy of rates of the single atom, cluster or molecular precursor displacements as compared to the deposition rate. Owing to the importance of kinetics, we focus in the rest of the chapter on the kinetic description of growth. In order to simplify the topic, we start with coverages below one atomic layer that is referred to as a monolayer. The first submonolayer part will be on nucleation, followed by a discussion of island shapes that, very much like snowflakes, tell us about the elementary processes that took place during their formation. We then discuss island coarsening, either by evaporation of atoms from their edges, referred to as the Ostwald ripening, or by the diffusion and subsequent coalescence of entire islands, referred to as the 
Smoluchowski ripening. We then turn to multilayers and discuss in analogy to submonolayers the growth morphologies and the atomic or molecular key processes giving rise to them. We finish with a brief discussion of growth manipulation by techniques mostly relying on kinetics that enable to induce the often-desired twodimensional (2D) growth. This chapter is a tutorial introduction to the subject; at the end, we recommend a few references for in-depth studies and further reading.

\section{1}

\section{Introduction}

The growth of thin epitaxial films is fascinating on its own sake. It is a challenge to identify the relevant atomic processes and to discover their interaction giving rise to a certain morphology. As these processes and their interplay will be the leading topic of this chapter, we start with a technological motivation outlining a few cases where the control of film growth has been mandatory.

20.1.1

\section{Technological Applications of Epitaxial Thin Films}

Our today's solid-state electronic devices rely on the growth of sequences of many ultrathin epitaxial layers with atomically sharp interfaces and thickness control down to the monolayer level. A typical example is the quantum well structures, where alternating layers of large- and small-band-gap semiconductors are grown to tailor the electronic properties by quantum confinement of charge carriers in the small-band-gap regions. Applications are ultrafast transistors, sensors, and semiconductor lasers. Examples of semiconductor lasers are quantum well and quantum cascade lasers [1-3]. These lasers can be tuned to the technologically relevant low-terahertz regime, where air has transmission windows [4-6].

An example of equal practical interest is stacking sequences of ferromagnetic layers separated by nonmagnetic spacers. Metallic spacer layers mediate magnetic coupling between subsequent ferromagnetic layers, and this coupling varies form ferromagnetic to antiferromagnetic with only a few monolayer spacer thickness. For antiferromagnetic coupling, one can realize a spin valve having strong-magneticfield-dependent electrical conductance [7, 8]. A magnetic field switches the layers from anti ferromagnetic to ferromagnetic alignment. Owing to strong spin scattering at the interfaces, the conductance between the layers across the spacer exhibits very large magnetoresistance, which is referred to as giant magnetoresistance (GMR).

For insulating spacers, the electrons tunnel between the ferromagnets. For well-suited wave function matching, this gives rise to tunnel magnetoresistances (TMRs) far beyond the GMR. TMR is used in magnetic hard-disk read heads and sensors. However, an even more promising application is the magnetic random access memories (MRAMs) that will possibly replace the current dynamic random access memories (DRAMs) because of their potentially much shorter access and write times, and because they are nonvolatile. Depending on cost, they may even 
replace hard-disk drives, or the current solid-state drives that are based on flash memories.

Whether MRAMs will be in our computers critically depends on the progress in epitaxial growth, as illustrated by the evolution of the achieved room-temperature TMR values over the years. Theory predicts that coherent and state-selective tunneling in fully epitaxial junctions may give rise to TMRs of the order of 1000\% [9, 10], that is, the resistance changes by a factor of 10 for parallel versus antiparallel magnetization. The first breakthrough in experimental junctions was achieved in 1995 when the TMRs went from former values of a few percent up to $18 \%$ in $\mathrm{Fe} / \mathrm{Al}_{2} \mathrm{O}_{3} / \mathrm{Fe}[11]$ and $12 \%$ in $\mathrm{CoFe} / \mathrm{Al}_{2} \mathrm{O}_{3} /$ Co tunnel junctions [12]. Another order of magnitude was achieved in 2004 when $188 \%$ TMRs were reported for fully epitaxial $\mathrm{Fe} / \mathrm{MgO}(100) / \mathrm{Fe}$ junctions [13] and $220 \%$ for polycrystalline $\mathrm{FeCo} / \mathrm{MgO} / \mathrm{FeCoB}$ junctions with (001) texture [14]. In 2005, finally, 230\% for CoFeB/MgO(100)/CeFeB junctions with polycrystalline ferromagnets, facilitating the fabrication of junctions with uniform and reproducible properties [15]. The current room-temperature record is $410 \%$ and realized in fully epitaxial $\mathrm{Co}(100) / \mathrm{MgO}(100) / \mathrm{Co}(100)$ junctions [16]. We note that not only the reading but also the writing of MRAMs will be based on a fundamental discovery, namely, the spin torque effect $[17,18]$, allowing the use of a spin-polarized current instead of a magnetic field to write the magnetic state of the memory cell [19].

In both examples, and actually in most of the technologically relevant cases, the growth of perfectly flat 2D layers of materials A/B, and subsequently B/A, is a stringent requirement for functionality. The rare examples where a threedimensional (3D) growth morphology is desired are semiconductor quantum dots, which are relevant for optoelectronic devices, single-electron transistors, or in heterogeneous catalysis and chemical sensors.

There are many more examples other than MRAMs, where the main reason why we do not have them yet in applications is that controlling their growth is rather challenging. For instance, graphene is predicted to be an ideal spin filter [20], yet defect-free sandwiches of ferromagnets with graphene have not been grown. Another compelling idea is the cold cathodes, where hot electrons can be created by a voltage applied to metal/insulator/metal junctions. One of the metal layers is made thinner than the ballistic mean-free electron path. Thus the electrons tunneling through the insulator travel ballistically through the top electrode where they are ejected into vacuum with an energy corresponding to the applied voltage minus the top-electrode work function. Such sources could promote chemical reactions on the top electrode [21] and be used in electron beam lithography $[22,23]$ or as electron sources in free-electron lasers.

A lot of interest has recently been attracted by the so-called multiferroics characterized by the coexistence of electric dipolar and magnetic order such that an electric field can be used to change the magnetic state. The applications for the most promising materials are the so-called artificial, or composite, multiferroics, where thin ferroelectric films are grown on ferromagnetic substrates -or the other way around [24]. An example is (La, $\mathrm{Sr}) \mathrm{MnO}_{3} / \mathrm{BaTiO}_{3}$ (LSMO-BTO) where an 
electric field applied to BTO can induce permanent changes in the magnetization of LSMO [25]. The growth of these systems poses real challenges.

A final example that is commonly used, and where crystallinity is not an issue, are multilayers grown as optical interference coatings, either for anti-reflection or as efficient mirrors for certain wavelengths. When these layers are intended especially for mirrors in the extended ultraviolet or soft X-ray range, the growth has to be controlled on an atomic layer basis [26].

The presented examples give a flavor why the growth of thin epitaxial films is technologically relevant. This chapter will now concentrate on the fundamental concepts.

\subsection{2}

\section{Epitaxial Thin Film Versus Crystal Growth}

The degree to which one is far from thermodynamic equilibrium distinguishes epitaxial thin film from crystal growth. This is illustrated in Figure 20.1 contrasting an artificial snowflake [27] with a natural quartz crystal. Both objects are single crystals; however, their shapes are quite different. The dendritic ice crystal in Figure 20.1a has preferred growth directions of its branches, shows self-similarity, and has a fractal dimension [28]. The $\mathrm{SiO}_{2}$ crystal in Figure 20.1b has very smooth facets by which it only exposes the energetically favorable low-index surfaces.

The dendritic ice crystal has been grown in very pure and uniformly undercooled water. It has been seeded by the solid phase growing inside the vertical capillary tube seen in the lower half of the photograph. Once the solid phase emerges through an orifice at the center of the container, it constitutes the nucleus on which the artificial snowflake then rapidly grows. The quartz crystal has been grown by many orders of magnitude slower in a natural geological process. Also, artificial crystals are grown very slowly such that the growing atomic or molecular species goes several times back and forth between the solid and the solution or gas phase before its definite condensation. This is different in (a) where the water molecules condense upon their first impact on the seed surface. Therefore, the quartz crystal

a)

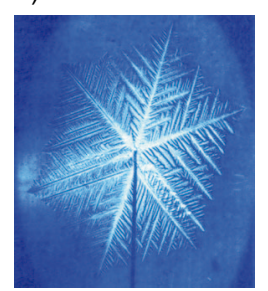

b)

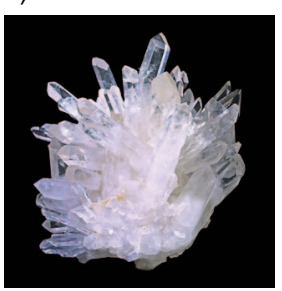

c)

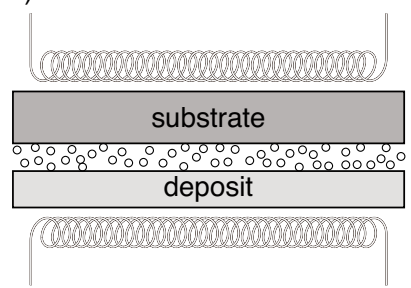

Figure 20.1 (a) Dendritic ice crystal grown in pure water at an undercooling of $-2.34{ }^{\circ} \mathrm{C}$. (Adapted from Ref. [27].) (b) Natural quartz crystal with large smooth facets (figure width 15 $\mathrm{cm})$. http://www.mineralminers.com. (c) Gedanken experiment illustrating the concept of supersaturation. (Adapted from Ref. [29].) 
is grown very close to thermodynamic equilibrium, while the artificial snowflake is grown very far from it.

Figure 20.1c shows a "Gedanken" experiment that is ideally suited to quantify the proximity to thermodynamic equilibrium [29]. The film material is evaporated from a deposit plate heated at the temperature $T_{\text {evap }}$ onto a parallel substrate plate residing at $T_{\text {subst }}$. The distance between the plates is smaller than the mean-free path of the evaporated atoms or molecules in the surrounding vacuum. The two temperatures define the respective vapor pressures $p$ and the ratio of their lograithms is called the supersaturation

$$
\sigma=\ln \left(\frac{p\left(T_{\text {evap }}\right)}{p\left(T_{\text {subst }}\right)}\right)
$$

This quantity is used in crystal growth to express the driving force for the solidification, as the chemical potential difference between the particles in the gas phase (or solution) and on the substrate is $\Delta \mu=\sigma k_{\mathrm{B}} T_{\text {subst }}$. This is the quantity that defines whether the particles touch the substrate and go back into the gas phase many times before they eventually stay or whether the first impact leads to their irreversible condensation.

For a typical thin film growth experiment on a Pt substrate with $T_{\text {sub }}=300 \mathrm{~K}$, the Gibbs free energy of evaporation yields $p_{\mathrm{Pt}}(300 \mathrm{~K})=10^{-90}$ mbar. For a deposition flux of $F=1 \mathrm{ML}$ (monolayer) $\mathrm{s}^{-1}$ one needs a deposit pressure of $p_{\text {evap }}=10^{-6}$ mbar. Thus, the supersaturation is $\sigma \approx 200$ and $\Delta \mu \approx 5 \mathrm{eV}$ atom $^{-1}$. Under these conditions, the visit of each substrate site, even of the ones with $1-2 \mathrm{eV}$ less binding energy than the ideal kink sites, will give rise to irreversible condensation. The diffusion barriers that the condensed atom encounters on its way to the ideal sites, and the resulting diffusion speed with respect to $F$, will decide whether it reaches the total energy minimum or gets trapped on metastable adsorption sites. The dendritic crystal morphology shown in Figure 20.1a is a typical growth shape resulting from the occupation of many metastable sites.

Crystal growth, on the contrary, takes place under supersaturations of $\sigma=1$ or below, leading to $\Delta \mu \approx 10 \mathrm{meV}$ atom $^{-1}$. Therefore, only the kink sites will give rise to condensation because for all other sites, it is favorable to return back to the gas phase or solution. Under these conditions, each crystal plane grows by step-flow growth, with very few defects, and typically starting from a screw dislocation because this avoids 2D nucleation [30]. The resulting shape is shown in Figure 20.1b. For many materials, such as artificial diamond, the growth of such large crystals remains challenging, while, triggered by Ronald Reagan's Star Wars Program, up to $1 \mathrm{~m}$ large organic single crystals have been grown with excellent quality.

20.2

Deposition Techniques

The deposition technique chosen for the epitaxial growth of a thin film depends on (i) the material to be grown and (ii) whether preference is given to crystallinity, 
thickness control, and thickness homogeneity; to stoichiometry control; or to large deposition rates. We focus here on the techniques that are not treated in other chapters of this volume, and the reader can refer to Chapter 23 for laser ablation, pulsed laser deposition (PLD), and plasma-assisted physical vapor deposition (PVD) and to Chapter 26 for organic film growth.

\subsection{1}

\section{Physical Vapor Deposition}

PVD is also referred to as molecular beam epitaxy (MBE) or atomic beam epitaxy (ABE), depending on whether molecules or atoms, respectively, are evaporated onto the substrate. In PVD, growth takes place under ultrahigh vacuum (UHV) conditions at a total pressure of $p_{\text {tot }} \leq 1 \times 10^{-8} \mathrm{~Pa}$. For the growth of metal oxides, one might also evaporate the metal atoms in a partial pressure of oxygen, or in general, one element is evaporated and a second is dosed from a partial pressure of molecules containing it.

From the following consideration, it becomes evident that the good pressure during deposition is mandatory for the cleanliness of the resulting films. From kinetic gas theory, one derives the flux $F$ of gas molecules with mass $m$ at pressure $p$ and temperature $T$ onto a surface

$$
F=\frac{p}{\sqrt{2 \pi m k_{\mathrm{B}} T}}
$$

As an example, we take $\mathrm{CO}$ molecules with a partial pressure of $p_{\mathrm{CO}}=1 \times 10^{-8} \mathrm{~Pa}$ at $300 \mathrm{~K}$. This yields an impingement rate per lattice site of a $\mathrm{Pt}(111)$ substrate (unit cell $A=6.65 \times 10^{-20} \mathrm{~m}^{2}$ ) of $\nu=F A=3.17 \times 10^{-6} \mathrm{~s}^{-1}$. If growth is performed at a deposition flux of $F=1 \times 10^{-3} \mathrm{MLs}^{-1}$, here we use the physical ML definition of one film atom per substrate atom, and if the sticking coefficient of $\mathrm{CO}$ is 1 , the residual gas will lead to $0.3 \%$ film impurities.

Another more restrictive consideration is the cleanliness required for nucleation studies or for a reproducible multilayer film morphology. Impurity atoms or molecules present on the substrate before evaporation of the film material will capture diffusing film atoms and lead to heterogeneous nucleation of islands. If the intrinsic island density to be studied is $n_{x}=1 \times 10^{-3} \mathrm{ML}$ (island densities $n_{x}$, with $x$ standing for an arbitrary size in number of atoms, are conveniently given in islands per substrate unit cell, i.e., in ML), and we tolerate a small fraction of islands created at impurities, then their density has to be $n_{\text {imp }} \leq 1 \times 10^{-5} \mathrm{ML}$. This value is reached in the above-mentioned example after $3.15 \mathrm{~s}$. Fortunately, many of the molecules in the residual gas have sticking coefficients much smaller than 1 for usual deposition temperatures. However, this clearly motivates to work at the lowest feasible pressures and to keep the time between substrate preparation and film deposition short. Total pressures of $p_{\text {tot }} \leq 3 \times 10^{-9} \mathrm{~Pa}$ can be achieved in regular UHV chambers, while cryopanels enable going below this value.

Film elements of low melting temperature and organic molecules are evaporated from Knudsen cells. They are composed of a crucible made of boron nitride (BN), 
refractory metal, or $\mathrm{Al}_{2} \mathrm{O}_{3}$. It contains the material to be evaporated and is closed toward the sample direction by an aperture, creating conditions where only single atoms or molecules and no clusters are evaporated. Further down the line toward the sample is a shutter. The evaporator shroud is water-cooled to keep desorption from its walls low. The deposition flux $F$ is adjusted by the crucible temperature; for the first order, one can refer to the respective vapor pressures of the elements or molecules to get an idea on the expected flux at a given temperature.

For refractive elements, one uses electron beam evaporators, where a filament surrounds a rod of the evaporation material, or a metallic crucible containing it. Thermally emitted electrons are accelerated to the rod or crucible, heating it with their kinetic energy in addition to the thermal radiation of the filament. Again, the chassis of these electron beam evaporators is water-cooled and a shutter determines the deposition time. The temperature of the evaporant is not directly monitored. However, the heating power is controlled by the high voltage and current between filament and target, and the flux can be monitored by measuring the current produced by the evaporation of charged atoms and by assuming that their fraction is flux independent. Depending on the relative potentials used between filament, rod, and sample, the charged species might acquire high kinetic energies and one might need to electrostatically deflect them for nucleation studies in order to avoid defect creation by sputtering or implantation.

Both types of evaporators are ideally outgassed, in addition to a thorough conventional degassing, by a final degas with open shutters and at deposition fluxes slightly above the ones used for film growth. This keeps the base pressure high during evaporation in the $1 \times 10^{-9} \mathrm{~Pa}$ range and codeposition of $\mathrm{H}_{2}$ and other molecules low. For some elements, such as Fe and Co, it is custom to leave the evaporators turned on, but at almost negligible flux. This keeps the evaporant at high enough temperature to inhibit adsorption of residual gas molecules onto it.

\subsection{2}

\section{Chemical Vapor Deposition}

In chemical vapor deposition (CVD), the growth chamber is filled with a partial pressure of a molecule containing the element or the molecular fraction to be deposited as film material. Again, cleanliness of the film is measured by the ratio of the partial pressures of the precursor molecules to the ones in the residual gas and their respective sticking coefficients. The essential differences with respect to PVD are the high substrate temperature used for CVD and the fact that the substrate catalyzes the dissociative chemisorption of the film material.

A topical example is the CVD growth of graphene on single-crystal metal surfaces, which we only briefly discuss here, as more details are given in Chapter 25 of this volume. The carbon containing molecules are, for example, ethylene [31-35] and ethene [36-38]. At most metal surfaces, $\mathrm{C}_{2} \mathrm{H}_{4}$ deprotonates to ethylidene $\left(\mathrm{C}_{2} \mathrm{H}_{3}\right)$ already at $300 \mathrm{~K}[31,39]$; above $430 \mathrm{~K}$, further dehydrogenation occurs, until, at $700 \mathrm{~K}$, only chemisorbed $\mathrm{C}$ atoms remain at the surface. From the C-adatomconcentration-dependent growth rate, there is evidence that graphene grows by the 
attachment of $\mathrm{C}_{5}$ rings [40-42]. For its growth on $\mathrm{Rh}(111)$, even larger building units of seven $\mathrm{C}_{6}$ rings have been proposed [38].

The crystalline quality of graphene is expressed by the density of chemical point defects and by the crystalline domain size. Both determine the electron scattering and phase coherence length, and thereby the transport and quantum mechanical coherence properties needed for applications, as well as for fundamental experiments. In many cases, graphene forms a moire pattern because of the lattice misfit with the substrate. These patterns can be mapped in scanning tunneling microscopy (STM) images that determine their point defect density and domain size. The latter is best quantified by the autocorrelation length. For best order, CVD has to be taken out at high $T$; however, for many metals, the $C$ solubility augments with increasing $T$. For $\mathrm{Ru}$, it is six times higher at $1540 \mathrm{~K}$ than that at $1000 \mathrm{~K}$ [44]. Therefore, one can load the near-surface region with C created by CVD and slowly surface-segregate this $\mathrm{C}$ by slow cooling, which leads to very good long-range order, as evidenced in Figure 20.2. Note that graphene can also be grown by MBE from high-purity $\mathrm{C}$ rods [40].

In general, CVD is a common and very versatile growth technique. It has been used for the growth of carbon nanotubes (CNTs) [45]. Multiwall CNTs are grown from acetylene at $600-800{ }^{\circ} \mathrm{C}$ and single-wall CNTs, due to their higher energy of

a)

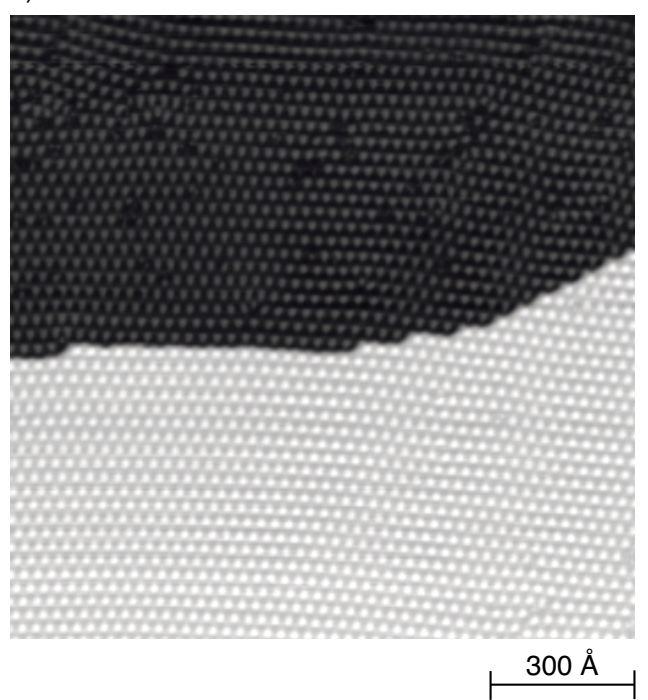

b)

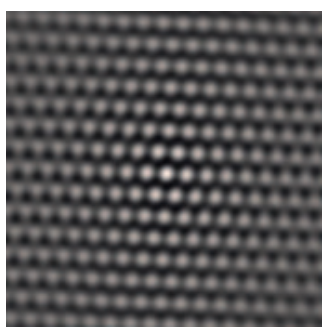

c)

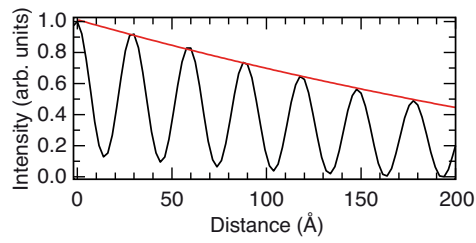

Figure 20.2 (a) Graphene layer on Ru(0001) obtained from dissolving carbon into the bulk during CVD and its subsequent surface segregation during slow cooling (exposure 127 Langmuir $\mathrm{C}_{2} \mathrm{H}_{4}$ at $T_{\text {CVD }}=1540 \mathrm{~K}$, cooling rate $\dot{T}=-0.1 \mathrm{Ks}^{-1}$ ). (b) $2 \mathrm{D}$ autocorrelation image of the STM image. (c) Linecut through (b) giving access to the autocorrelation length of $238 \pm 12 \AA$. (Adapted from Ref. [43].) 
formation, from $\mathrm{CO}$ and methane at $900-1200^{\circ} \mathrm{C}$ [46]. CVD is further used for $\mathrm{BN}$ growth, where typically borazene molecules are used and dehydrogenated on metals [47]. Finally, CVD is frequently used as an alternative to MBE for the growth of semiconductor thin films.

\subsection{3}

\section{Atomic Layer Deposition}

Atomic layer deposition (ALD) [48-50] is a variant of CVD. Its distinct feature is that films are grown in a cyclic manner by alternating between two or more precursor molecules that exhibit self-limiting surface reactions on the substrate.

Figure 20.3a illustrates one cycle for the example of $\mathrm{Al}_{2} \mathrm{O}_{3}$ growth. It starts with a partial pressure pulse of precursor $\mathrm{A}$, in our example $\mathrm{AlCl}_{3}$, to create a chemisorbed monolayer of $\mathrm{AlCl}_{2}$ on the substrate, followed by a purge of the deposition chamber removing unreacted precursor molecules A. Subsequently, a pressure pulse of precursor B creates a chemisorbed monolayer of B on top of A. In our example, precursor $\mathrm{B}$ is water and its adsorption leads to the formation of $\mathrm{Al}_{2} \mathrm{O}_{3}$. Remaining unreacted water molecules, as well as $\mathrm{ClH}_{n}$ species, are removed from the surface and the reactor's residual gas by a purge closing the deposition cycle. Figure 20.3b shows a cross-sectional SEM image revealing, the high conformality of the coating, in particular also of the high-aspect-ratio surfaces, because the precursor molecules can reach the hidden surface areas and react there with equal rates as at substrate protrusions.

Depending on the precursors, a large variety of materials can be deposited, including various oxides, nitrides, or metals. The growth cycles, typically a few seconds each, are repeated as many times as required for the desired film thickness. The film growth is self-limiting; that is, the amount of film material deposited in each reaction cycle is constant and very close to $1 \mathrm{ML}$ of the film material.

While the principle of ALD was invented some decades ago [48, 49], its full potential in nanotechnology was realized only much later [51, 52], and only recent applications in semiconductor industry have boosted its widespread commercial use. The main advantages of ALD are (i) the high conformality, that is, uniform

a)

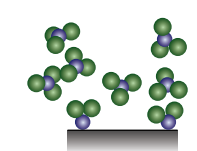

$\mathrm{AlCl}_{3}(\mathrm{~g})$

Pulse

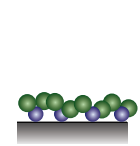

$\mathrm{AlCl}_{2}(\mathrm{~s})$

Purge

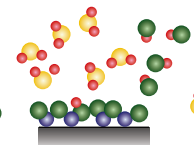

$\mathrm{H}_{3} \mathrm{O}(\mathrm{g})$

Pulse

Repeat ALD cycle $\mathrm{N}$ times

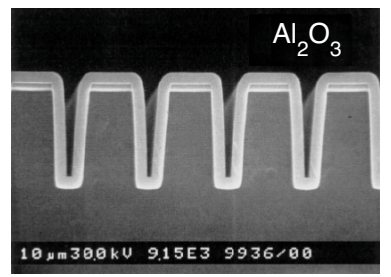

Figure 20.3 (a) ALD process steps illustrated for the deposition of $\mathrm{Al}_{2} \mathrm{O}_{3}$ from the precursors $\mathrm{AlCl}_{3}$ and $\mathrm{H}_{2} \mathrm{O}$. (b) Cross-sectional SEM image of $\mathrm{Al}_{2} \mathrm{O}_{3}$-coated high-aspect-ratio mask revealing excellent conformality. 
coatings can be deposited on complex shapes, structured surfaces, as well as on both sides of a substrate and simultaneously on a large number of them; (ii) the films are very dense and have minimal porosity, in particular, no pin holes; (iii) dopant materials can be added to the film in a controlled way and mixtures of two or more film materials can be deposited. Often ALD-grown films are amorphous; however, this technique can equally be used to create crystalline epitaxial films.

In most ALD systems, the surface reactions are enabled by thermal activation, meaning that the substrate is kept at typically $200-400^{\circ} \mathrm{C}$. More advanced systems are plasma assisted, that is, they employ plasma species as reactants during one or more steps of the cyclic deposition process. This allows for more freedom in processing conditions and for a wider range of material properties. This is especially beneficial for the growth of metal films, where conventional thermally driven ALD is limited by significant nucleation delay, which can inhibit growth for the first 100 growth cycles or more [53]. Moreover, high substrate temperatures can be avoided, increasing the range of substrate materials that can be coated.

20.2.4

\section{Electrochemical Deposition}

We discuss the principles of electrochemical deposition (ED) in the case of metals. Metal atoms are solvated in the electrolyte either as complexes or as metal cations. The metal cations are electrochemically reduced at the substrate/electrolyte interface according to the generic reaction $\mathrm{M}^{z+}+z \mathrm{e}^{-} \rightarrow \mathrm{M}$. A sketch of the electrochemical cell with this reaction taking place is shown in Figure 20.4a. The first electrode is the substrate onto which the metal atoms $\mathrm{M}$ are deposited; the second is the reference electrode (RE), typically a mercury sulfate electrode

a)

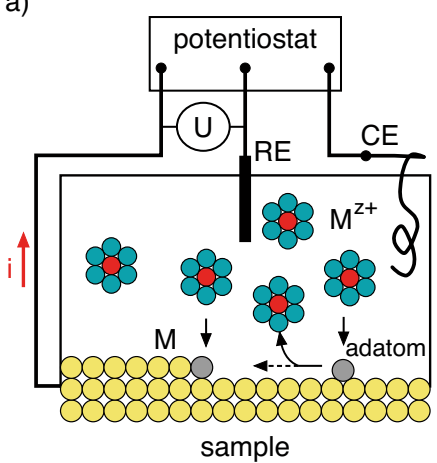

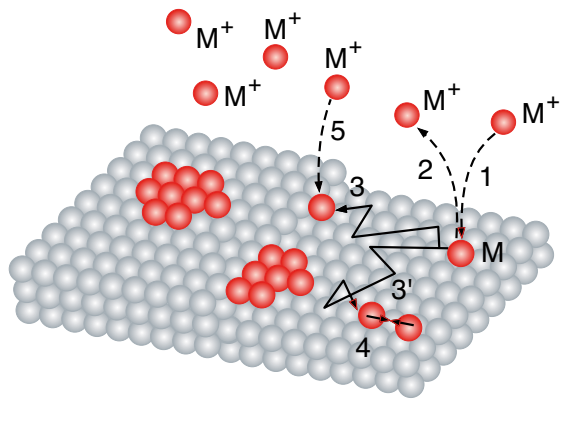

Figure 20.4 (a) Electrochemical cell with electrodes: substrate (sample), reference (RE), and counter electrode (CE). Metal cations in solution (red) with solvation shell (blue) that is removed on reduction and deposition (redgray). (b) Atomic processes of electrochemical metal deposition. Here $M$ atoms are drawn in black, irrespective of whether they are in solution or on the substrate (Adapted from Ref. [54].) 
probing the electrolyte potential without current flow; and the third is the counter electrode (CE) over which the electrochemical current between electrolyte and substrate flows.

The applied voltage $U$ controls whether deposition or dissolution take place and determines the rate of the respective reaction. One introduces the overpotential $\eta=\Phi-U$, with $\Phi$ being the Nernst equilibrium potential of the redox couple $\mathrm{M}^{z+} / \mathrm{M}$. ED requires $\eta>0$ and is therefore also referred to as overpotential deposition $(O P D)$. As the reaction is thermally activated, the deposition flux is given by an Arrhenius expression, $F \propto\left(\mathrm{e}^{\alpha z q \eta / k_{\mathrm{B}} T}-1\right)$, where $q$ is the elementary charge and $k_{\mathrm{B}}$ is the Boltzmann constant [54-56]. The transfer coefficient $\alpha \approx 0.5$ is related to the profile of the potential barrier across the electrolyte/substrate interface. The expression for $F$ is called the Butler-Volmer equation and valid until a threshold value of $\eta$ where the reaction rate gets limited by the cation concentration and electrolyte convection.

In analogy to PVD, there is a flux of incoming film atoms, step 1 in Figure 20.4b, followed by diffusion over the substrate (3). However, in contrast to PVD, the adsorption involves the discharge of the metal cation by capture of electrons from the substrate, and more importantly, the rate of desorption back into the solution (2) is generally much larger than that at typical substrate temperatures used in PVD. If the desorption potential is sufficiently close to $\Phi$, one may reach in ED very large degrees of incomplete condensation and thus approach thermodynamic equilibrium, where in analogy to the Gedanken experiment discussed previously many sites are probed and only the ones with the largest binding energy, such as kinks (5), lead to irreversible condensation. Homogeneous adatom nucleation at terraces (4) and their incorporation into steps (3) are competing processes of condensation.

In some cases, deposition is observed also for $\eta<0[57,58]$. This underpotential deposition (UPD) implies the formation of a single monolayer of metal atoms and metal anions and is self-limiting, that is, the deposition is terminated after completion of this layer. The nature of the anions may greatly influence whether and how UPD takes place [58]. Note that atomic details of ED can be observed in situ by means of electrochemical STM for both ED modes [54-62]. For semiconductor and oxide ED, the reader can refer to Refs [63-65].

\section{3}

\section{Growth Modes}

By the middle of the twentieth century, the understanding of epitaxial growth was divided into three major schools of thought. Frank and van der Merwe used elasticity theory to derive the concept of a critical misfit below which monolayerby-monolayer growth appears [66]. Volmer and Weber [67], applying nucleation theory, assumed that crystalline films grew from 3D nuclei on the substrate and that their relative number and growth rate were determined by interfacial and surface free energies. The third model by Stranski and Krastanov [68] was based on 
atomistic calculations and assumed that initially a few pseudomorphic 2D layers are formed, on top of which 3D crystals with their natural lattice constant will grow. Each of the three scenarios is observed, thus the three schools complement each other; they gave rise to the following labeling of the three growth modes of epitaxy:

- The Frank-van der Merwe (FM) growth mode (2D morphology, layer-by-layer, or step-flow growth).

- The Volmer-Weber (VW) growth mode (3D morphology, island growth).

- The Stranski-Krastanov (SK) growth mode (initially 2D, after critical thickness, 3D morphology, layer-plus-island growth).

Unification of the three historical approaches to epitaxy and prediction of the growth mode were achieved by Bauer in considering the thermodynamic quantities involved in epitaxy, namely, the three macroscopic surface tensions: $\gamma_{0}, \gamma_{i}$, and $\gamma_{s}-$ the free energy per unit area at the overlayer/vacuum interface, the overlayer/substrate interface, and the substrate/vacuum interface, respectively [69]. Bauer's concept is evident by comparing Figure 20.5a and b. If $\gamma_{0}$ and $\gamma_{i}$ are small compared to $\gamma_{\mathrm{s}}$, the system gains energy when being totally covered by the overlayer (the FM growth mode), whereas, if not, it will only partly be covered (the VW growth mode). For a film composed of $n$ layers, the criterion of the FM growth mode is

$$
\gamma_{\mathrm{o}(n)}+\gamma_{\mathrm{i}(n)} \leq \gamma_{\mathrm{s}}
$$

a)

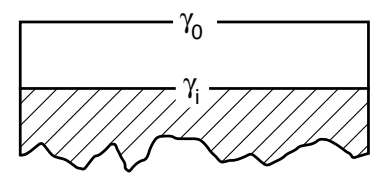

FM

c)

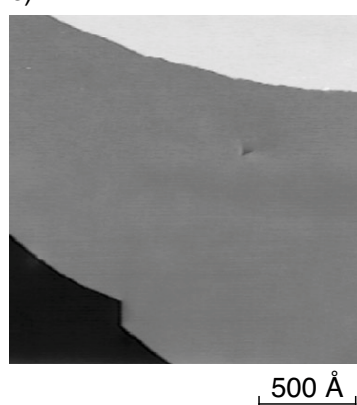

b)

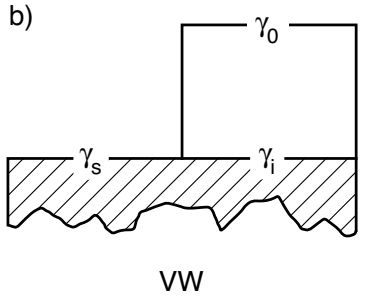

d)

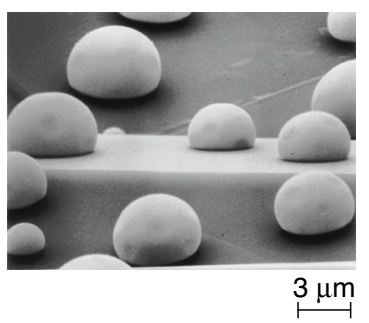

$3 \mu \mathrm{m}$

Figure 20.5 (a) Frank-van der Merwe (FM) growth compared with (b) Volmer-Weber (VW) growth [70]. (c) Perfectly 2D Ag film with $\mathrm{Ag}(111)$ lattice constant grown by depositing $25 \mathrm{ML} \mathrm{Ag}$ onto $\mathrm{Pt}(111)$ at $600 \mathrm{~K}$ and subsequent annealing to $800 \mathrm{~K}$ [71]. (d) 3D Pb islands grown on highly oriented pyrolytic graphite (HOPG) imaged with a SEM. (Kindly provided by J. J. Métois.) 
The $n$ dependence of $\gamma_{0}$ reflects surface free energy changes caused by possible changes in lattice constant and/or structure at the overlayer surface. The thickness dependence of $\gamma_{i}$ has its origins in (i) the true interface energy caused by the generally different crystallographic structure and/or lattice constant of overlayer and substrate and by the electronic hybridization at the interface and (ii) the volume strain accumulated in a pseudomorphic overlayer that is conveniently incorporated into $\gamma_{\mathrm{i}(n)}$ [72]. The equality in Equation 20.3 holds for the trivial case of homoepitaxy that thus displays the FM growth mode, if grown under conditions close to thermodynamic equilibrium. In the heteroepitaxial case, the obvious condition for the FM mode is that $\gamma_{o(n)} \ll \gamma_{\mathrm{s}}$. The inequality in Equation 20.3 has to be large because, in general, $\gamma_{\mathrm{i}(n)}$ is positive and nonnegligible. The VW mode results if $\gamma_{\mathrm{o}(n)}>\gamma_{\mathrm{S}}$.

Examples of the FM and VW growth modes are shown in Figure 20.5c,d. A 25 ML thick Ag film on Pt(111) grows perfectly flat, as evidenced by the STM image showing extended atomically flat terraces separated by monoatomic steps. The reason is that the surface free energy of $\mathrm{Ag}(111)$ is with $\gamma_{\mathrm{o}}=1.17 \mathrm{~J} \mathrm{~m}^{-2}$ small compared to that of $\mathrm{Pt}(111), \gamma_{\mathrm{s}}=2.30 \mathrm{~J} \mathrm{~m}^{-2}$, and the strain containing interface energy of $25 \mathrm{ML} \mathrm{Ag/Pt}(111)$ is sufficiently small. The VW growth mode is realized for $\mathrm{Pb}\left(\gamma_{\mathrm{o}}=0.60 \mathrm{~J} \mathrm{~m}^{-2}\right.$, for a survey of metal surface free energies see Ref. [73]) on graphite $\left(\gamma_{\mathrm{s}}=0.077 \mathrm{~J} \mathrm{~m}^{-2}\right.$, at $1243 \mathrm{~K}$ [74]) as revealed using scanning electron microscopy (SEM). If one is close to thermodynamic equilibrium, such that the $3 \mathrm{D}$ island shape is comparable to that of liquid $\mathrm{Pb}$ droplets, the ratio of the surface free energies of $\mathrm{Pb}$ and HOPG (highly oriented pyrolytic graphite) is reflected in the contact angle $\Theta$ formed by the $\mathrm{Pb}$ cluster surface and the substrate [75]

$$
\cos \Theta=\frac{\gamma_{\mathrm{s}}-\gamma_{\mathrm{i}}}{\gamma_{\mathrm{o}}}
$$

Lattice mismatch of overlayer and substrate leads to a monotonic increase in volume strain energy in a 2D pseudomorphic film with increasing thickness $n$. Equation 20.3 implies an instability at a critical thickness $n_{\mathrm{c}}$, where $\gamma_{\mathrm{o}(n)}+\gamma_{\mathrm{i}(n)}>\gamma_{\mathrm{s}}$, and the system switches from 2D to 3D growth morphology. This SK mode presents a significant issue in the fabrication of coherently strained 2D device structures. On the other hand, the strain-driven morphology transition is beneficial for the selfassembly of quantum dots into arrays with sharp size and distance distributions, see Chapter 29 in this volume.

The equilibrium structure and morphology of a heteroepitaxial thin film are determined by the competition between the film-substrate interaction and the lateral adatom interaction in the film, describing the anisotropy of chemical bond strength parallel and perpendicular to the interface. Usual measures of these quantities are the isosteric heat of adsorption $V_{0}$ and the lateral adatom attraction $e_{1}$. The "phase diagram" derived from molecular dynamics simulations displayed in Figure 20.6a reveals the expected growth modes in the $V_{0} / e_{1}$ versus misfit $m$ plane, $m$ is defined by $m=\left(a_{\text {film }}-a_{\text {sub }}\right) / a_{\text {sub }}$. The diagram shows that one can stabilize the FM growth mode only for the so-called strong substrates, which impose their lattice constant onto the film atoms by a large corrugation of the 
a)

Frank - Van der Merwe

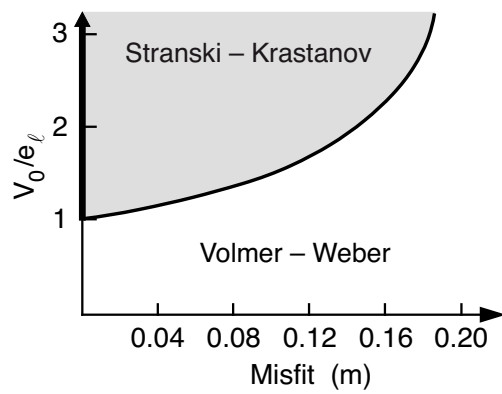

b)
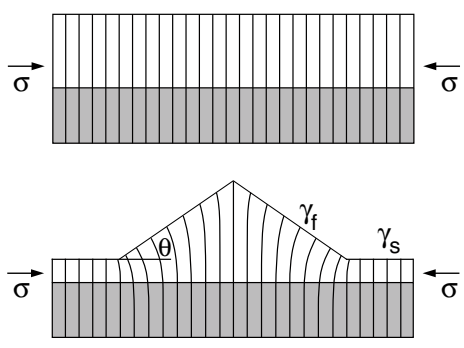

c)

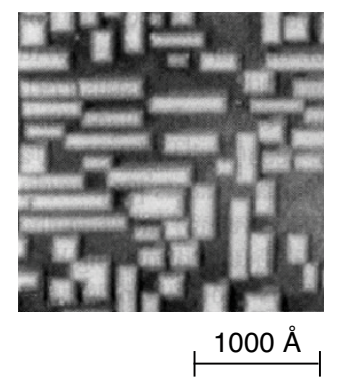

d)

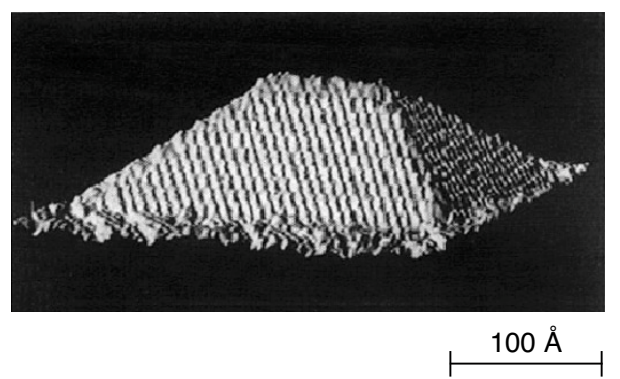

Figure 20.6 (a) Phase diagram of growth mode on an $\mathrm{fcc}(100)$ surface in the substrate strength $V_{0} / e_{1}$ versus misfit $m$ plane [76]. (b) Coherently strained 2D film versus 3D pyramidal island situated on top of a planar wetting layer. The contact angle and surface energy of the inclined facet are $\Theta$ and $\gamma_{f}$, respectively, while $\gamma_{s}$ denotes the surface energy of the wetting layer. The compressive misfit stress is $\sigma$ [77]. (c,d) STM images of "hut clusters" formed by the StranskiKrastanov growth of $\mathrm{Ge}$ on $\mathrm{Si}(100)$ [78].

interaction potential as compared to the stiffness of the adsorbate's lateral bonds $\left(V_{0} / e_{1}>1\right)$, and for small misfits. Films grow in the VW mode down to very small misfits when lateral interactions dominate $\left(V_{0} / e_{1}<1\right)$. If, for a given misfit, the influence of the substrate is increased, one passes from the VW to the SK mode, where a few 2D layers become thermodynamically stable.

There are two possible reasons for the transition from 2D to 3D morphology in the SK growth mode. First, the film material can grow in the first few monolayers in a crystallographic structure that differs appreciably from its own bulk, see, for example, fcc Fe stabilized on $\mathrm{Cu}(100)$ [79-81]. In this case, the SK mode will be accompanied by the crystallographic change to the film's bulk lattice structure taking place at $n_{\mathrm{c}}$. This induces an abrupt increase in free energy at the interface between the two crystal structures and shifts the energy balance in favor of 3D growth. The second possible reason is strain relief by the formation of "mounds." Figure 20.6b shows how mounds can adopt very efficiently to their bulk lattice constant in case of compressive strain. When the strain energy is high, the increase in surface area is more than counterbalanced. Figure 20.6c,d show STM images of faceted pyramids forming in the $\mathrm{Ge} / \mathrm{Si}(100)$ system that 
has $4.2 \%$ lattice misfit. Compared to mounds, it is much harder to relief strain in a perfectly 2D layer. In the most lucky cases, strained epitaxial layers exhibit a smooth transition in lattice constant from the substrate value over several weakly incommensurate phases (moiré structures or networks of partial surface dislocations) toward its bulk value [82]. Often, however, strain relief in a 2D layer implies the introduction of structural defects [83] such as bulk dislocations, which generally hamper functionality. Surface ripples [84] are an alternative way to relieve strain. With the increasing trend to use more highly strained material combinations, as InGaAs/GaAs and $\mathrm{SiGe} / \mathrm{Si}$, the suppression of the SK growth mode and the creation of defect-poor 2D layers of strained materials remains an important albeit largely unsolved issue. One promising way is to grow on micromachined substrates, as demonstrated by dislocation-free Ge crystals grown on $2 \mu \mathrm{m}$ wide and $8 \mu \mathrm{m}$ tall Si pillars [85].

\section{4}

Nucleation

The above-mentioned concept of growth modes is based on thermodynamic considerations. It is valid only when growth takes place close to equilibrium such that the morphology is given by the total energy, irrespective of the path taken by the film atoms in order to reach the minimum energy morphology. As discussed earlier, in many cases, the growth of epitaxial films is performed far away from equilibrium and the energy barriers encountered by the film atoms along their migration path on the surface matter. They define the diffusion rates and thus the kinetics of growth. The ensemble of barriers separates the manifold of all possible film morphologies. They will never be exactly the same but exhibit common features. Kinetics has advantages and drawbacks. It helps to realize thermodynamically unstable morphologies, but it might also inhibit reaching the thermodynamic equilibrium morphology, even after postannealing of the film. The nucleation of islands in the middle of atomically flat terraces is the most central kinetic process and will be discussed in this section. For simplicity, we concentrate ourselves on metals, as there the diffusing species are single atoms; however, the presented concepts can straightforwardly be enlarged to the growth of systems with more directional bonds, as, for example, semiconductors or the $\mathrm{sp}^{2}$-hybridized monolayers graphene or hexagonal BN $(h$-BN). We start by a description of the atomic processes involved in the growth of epitaxial thin films and then discuss homogeneous and heterogeneous nucleation.

20.4.1

\section{Atomic Processes}

Figure 20.7 shows the elementary atomic diffusion processes involved in atomic beam epitaxy. Film atoms are deposited onto the substrate with thermal energy $(\sim 0.1 \mathrm{eV})$ and flux $F$ expressed in monolayers per second, that is, in film atoms per 


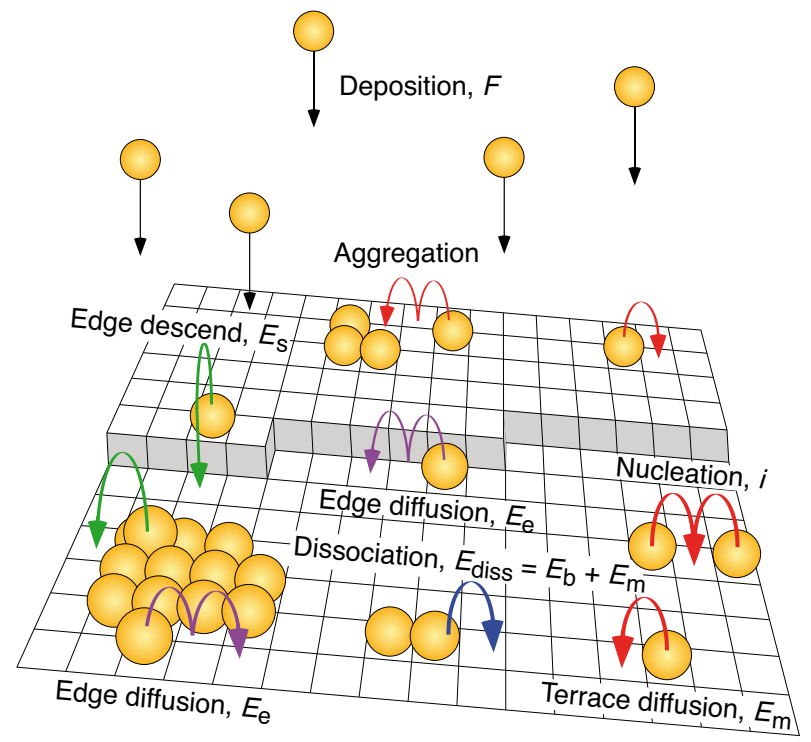

Figure 20.7 The elementary atomic diffusion processes of epitaxial thin film growth.

substrate adsorption site and per second. Somewhat surprisingly, the energy gained in the adsorbate-substrate bond formation is immediately dissipated to the lattice, so that adatoms are brought into thermal equilibrium, with the substrate already at their site of impact [39]. This can be inferred, for instance, from experiments at low temperatures revealing cluster sizes, or atom adsorption sites, in agreement with the predictions of statistical growth, allowing no diffusion at all [28, 86-88]. Therefore, transient jumps, where part of the adsorbate binding energy is used for diffusion, are the exception, if they exist at all for metal-on-metal adsorption. Transient nonthermal motion has to be distinguished from thermal motion with small energy barriers toward next nearest neighbors [89] and clusters [90, 91]. For certain combinations of elements, the adsorption energy might be used to trigger transient exchange processes, even at low $T$ [92]. However, in the absence of exchange and sufficiently far away from clusters or adatoms, the film atoms generally adsorb where they land from the vapor phase. From there on, adatom diffusion sets in.

The diffusion processes participating in epitaxial growth are thermally activated jumps, mostly in the form of single adatom movements between adjacent lattice sites. However, concerted motion of several atoms may also be involved. Transitionstate theory (TST) [93] assumes that the atoms stay between two subsequent jumps long enough in their adsorption wells to thermally equilibrate. In addition, recrossing of the saddle point is assumed to be negligible. These assumptions are justified if the energy difference separating the binding from the transition site that is passed upon diffusion satisfies $E \ll k_{\mathrm{B}} T$. The jump rate of a diffusion process of type $n$ is then given by the Boltzmann statistics as $\nu_{n}=v_{0, n} \exp \left(-E_{n} / k_{\mathrm{B}} T\right)$, with the attempt frequency $v_{0, n}$ typically being in the range of the Debye frequencies of $10^{12}-10^{13} \mathrm{~Hz}$. 
Terrace migration of single adatoms $\left(E_{\mathrm{m}}\right.$ in Figure 20.7) is the most fundamental of these diffusion processes. It gives rise to nucleation of islands on substrate terraces, or to step-flow growth at elevated temperatures, where the mean-free diffusion path of the adatoms is larger than the terrace width. Depending on the density of simultaneously diffusing particles, one distinguishes the collective diffusion coefficient (also known as chemical or the Fickian diffusion coefficient) of an ensemble of mutually interacting particles from the tracer (or intrinsic) diffusion coefficient describing the mean square displacement of one isolated random walker per unit time [94] (see also Chapter 8 in Volume 2). For typical growth rates, the density of diffusing particles is rather small (the monomer density $n_{1} \ll 10^{-3} \mathrm{ML}$, also densities are expressed in ML units, i.e., in islands or here adatoms per substrate unit cell). The mean interparticle distance is therefore large compared to typical interaction ranges for metal adatoms on metal substrates $[95,96]$ and cluster densities are determined by the tracer diffusion coefficient $D$ defined as

$$
D=D_{0} \exp \left(\frac{-E_{\mathrm{m}}}{k_{\mathrm{B}} T}\right) \text {, with } D_{0}=\frac{1}{4} \nu_{0}
$$

where $D$ is expressed in substrate unit cells per second. The factor of $1 / 4$ in $D_{0}$ is valid for 2D diffusion on a square lattice, as in Figure 20.7.

Diffusion across the terrace ends when the adatoms collide with one or more of their own, or when they hit a structural or chemical defect. If these defects are very sparse, or if they do not interact with the diffusing species, the island densities are dominated by the first two processes and one speaks about homogeneous nucleation. If the defects are more abundant than the homogeneous island density, and if they interact strongly with the diffusing film atoms, one speaks about heterogeneous nucleation, see the following for a more detailed discussion of the two cases.

For homogeneous nucleation, the formed clusters may either remain stable, or decay again. This depends on the lateral bond energy, $E_{\mathrm{b}}$ in Figure 20.7, and the number of neighbors. A stable nucleus is a cluster that is large enough to grow more rapidly than it decays on the timescale of deposition. One defines the critical cluster size $i$ by the number of atoms in the smallest stable nucleus minus one, that is, attachment of one more atom turns a critical cluster into one that stays stable until the next atom arrives.

The 2D cluster shape is determined by the mobility of aggregating adatoms along the cluster edge (barrier $E_{\mathrm{e}}$ in Figure 20.7). This is not a single barrier, as diffusion can take place along straight steps, around kinks and corners, and in the case of substrates with trigonal symmetry, also from onefold-coordinated corner to twofold-coordinated step sites. Low mobility leads to ramified clusters with fractal dimension, similar to diffusion-limited aggregation (DLA) scenarios [97-99] and in analogy to the ice crystal shown in Figure 20.1a with the difference that it occupies 3D space, whereas here the island shapes are restricted to a surface. Anisotropic terrace diffusion imposed by the substrate symmetry, in conjunction with anisotropic sticking to the edges, can lead to the formation of 1D monoatomic wires. Compact 2D clusters are created if edge diffusion is fast compared to 
the cluster growth rate. The thermodynamic 2D equilibrium shape forms when corner and kink crossing or even evaporation of step atoms to the terrace becomes activated. We discuss these cases in Section 20.5.

A very important atomic process for the morphology of films is adatom descent at island steps. In analogy to corner crossing in two dimensions, determining whether steps are straight or rough, interlayer diffusion determines whether films are flat or 3D. Its rate determines whether adatoms deposited onto the island top are able to descend to the substrate level before other adatoms become deposited onto the island top. If they cannot descend before, they may diffuse toward the other adatoms, and in the case of $i=1$, a stable nucleus forms on top of the island giving rise to the kinetic growth of 3D clusters. If they can descend sufficiently fast such that there is at most one adatom present on top of the island, the islands remain $2 \mathrm{D}$ until they coalesce, giving rise to layer-by-layer growth with 2D film morphology.

An adatom approaching a descending step encounters a barrier $E_{\mathrm{S}}$ for descent that is typically larger than $E_{\mathrm{m}}$. The extra diffusion barrier $\Delta E_{\mathrm{s}}=E_{\mathrm{s}}-E_{\mathrm{m}}$ to overcome atomic steps was experimentally discovered [100] and theoretically proposed [101] long ago. It is caused by the reduced substrate coordination of the adatom in the transition state where it diffuses down the step. Interlayer diffusion may also take place by exchange, where the lower-step atom is pulled out of the step and the upper atom enters into its site. In that case, $\Delta E_{\mathrm{S}}$ is caused by the reduced coordination of the more complex transition-state configuration.

$A b$ initio calculations give insight into why exchange diffusion is preferred for specific step orientations and combinations of elements, whereas for other step orientations and systems, interlayer diffusion is a simple "roll-down" process. The values for $\Delta E_{\mathrm{s}}$ derived from such calculations [102-104] can be compared to atomic-scale field ion microscopic (FIM) observations [39, 105]. Experiment and theory agree that the mechanism of interlayer diffusion is strongly system specific. Complementary to direct FIM inspection, there are also various indirect ways to infer experimental estimates on $\Delta E_{\mathrm{s}}$, for example, from layer occupation numbers [106, 107], from the nucleation probability on top of islands [108-111], from island decay in suitable geometries [112], or from step densities [113, 114] and slopes of mounds evolving through kinetic roughening $[115,116]$.

The values of $\Delta E_{\mathrm{s}}$ derived from such observations of the film morphology are all effective barriers for interlayer diffusion, $\Delta E_{\mathrm{s}, \text { eff }}$. Despite their relevance to predict the epitaxial growth morphology and cluster dimension, association to a particular microscopic interlayer diffusion process, for example, for comparison with theory, is often not unambiguously possible.

\subsection{2}

\section{Homogeneous Nucleation}

We will now address the relationship between cluster density, terrace diffusion coefficient, cluster binding energy, and deposition flux for homogeneous nucleation of 2D islands. We discuss the irreversible growth regime, where the critical cluster 
is a monomer $(i=1)$, and a dimer is stable and immobile. For an extension of this discussion to more complicated cases of larger critical cluster sizes, 3D clusters, incomplete condensation, where part of the deposited film atoms desorb again, or cluster diffusion, we refer to [117-120].

In the initial phase of deposition, the adatoms arriving from the gas phase with rate $F$ diffuse on the substrate terraces with rate $D$, defined by Equation 20.5 , until they meet a second diffusing adatom and create a dimer. As deposition proceeds, the number of dimers will increase linearly until their density $n_{2}$ becomes comparable to $n_{1}$. From there on, the probabilities that a diffusing monomer encounters one of its own or a dimer become comparable and the growth of stable clusters starts to compete with the nucleation of new ones. As a consequence, the increase in density of stable nuclei $n_{x}$ ( $x$ standing for any size that is stable, $x \geq 2$ ) levels off until it saturates at a coverage of typically $\Theta_{\text {sat }} \sim 0.15 \mathrm{ML}$. At that point, the mean-free path of diffusing adatoms is equal to the mean island separation and any further deposition will exclusively lead to island growth because all adatoms reach existing islands to which they attach. At coverages beyond $0.20 \mathrm{ML}$, the $2 \mathrm{D}$ clusters start to coalesce until the monolayer film percolates at typically $\Theta=0.55 \mathrm{ML}$. Experimental evidence for this scenario has been provided for $\mathrm{Ag} / \mathrm{Pt}(111)$ by means of variable-temperature (VT)-STM [121]. The STM observations ranged from the pure nucleation phase with its linear increase of $n_{x}$ and an average cluster size of only two to three atoms up to saturation and finally coalescence. Figure 20.8 shows monolayer-high $\mathrm{Ag}$ clusters formed at three deposition temperatures, each time at a coverage of $\Theta=0.12 \mathrm{ML}$ close to saturation and in the irreversible growth regime. One sees that the saturation island density strongly decreases with increasing deposition temperature.

For simplicity, we label the saturation island density $n_{x}$, as we did before for the density of stable nuclei at any coverage. Mean-field nucleation theory relates $n_{x}$ to the ratio of diffusion $D$ to deposition rate $F$ and to the cluster binding energy $E_{i}$ by the following expression for complete condensation and 2D clusters [117, 122]

$$
n_{x}=\eta(\Theta, i)\left(\frac{D}{F}\right)^{-\chi} \exp \left(\frac{E_{i}}{(i+2) k_{\mathrm{B}} T}\right) \text {, with } \chi=\frac{i}{i+2}
$$

From Equation 20.6, it becomes clear that the most direct link between $n_{x}$ and $D$ is obtained at low temperatures where $i=1$. Then the cluster binding energy is by definition $E_{i}=0$, and Equation 20.6 reduces to

$$
n_{x}=\eta(\Theta, 1)\left(\frac{D}{F}\right)^{-\frac{1}{3}}
$$

with $\eta(\Theta, 1)=0.25$ in the coverage range of saturation [88, 117]. At higher temperature, the cluster binding energy $E_{i}$ can, for instance, be expressed in a pair binding model involving multiples of the energy per bond $E_{\mathrm{b}}[118]$ (for dimers $E_{i}=E_{\mathrm{b}}$, see Figure 20.7).

The Arrhenius plot of $n_{x}$ in Figure 20.8d shows a linear regime in the range $10^{5} \leq$ $D / F \leq 10^{9}$, in accordance with Equation 20.7. The application of this equation to $n_{x}(T)$ data inferred from STM has proved to yield precise numbers for the barrier 
a)

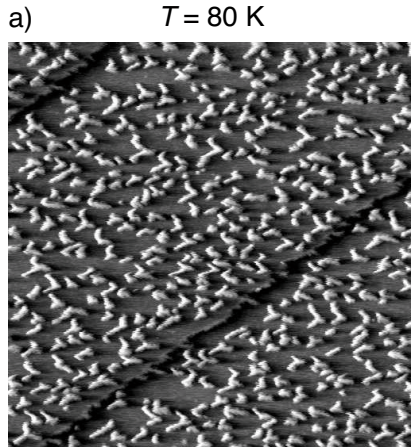

c)

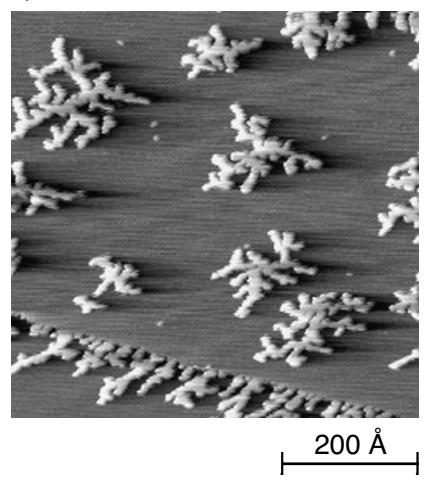

b)

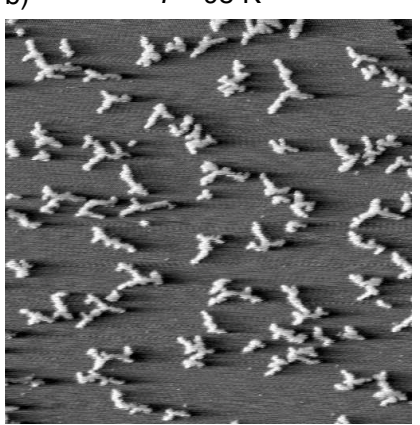

$D / F$

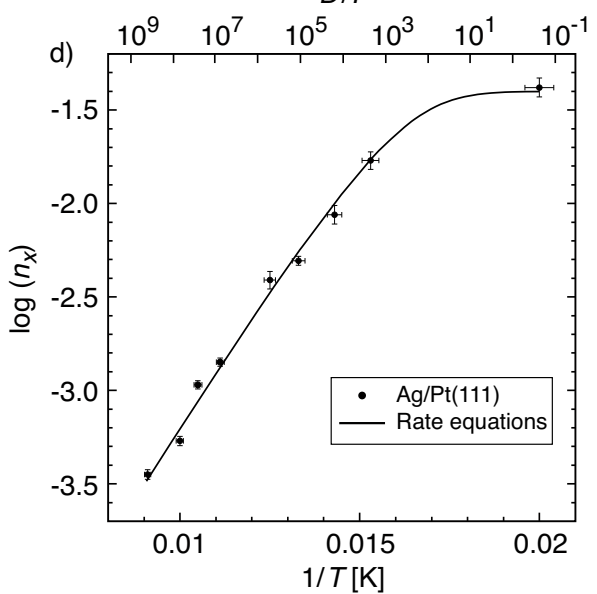

Figure 20.8 Variation of saturation island density with temperature for deposition of $\Theta=0.12 \mathrm{ML}$ $\mathrm{Ag}$ onto a Pt(111) surface. (a-c) Common length scale VT-STM images taken at the respective deposion temperatures. (d) Arrhenius plot of the saturation island densities in the temperature regime where dimers are stable nuclei $\left(F=1.1 \times 10^{-3} \mathrm{ML} \mathrm{s}^{-1}\right)$. Experimental island densities for $\mathrm{Ag} / \mathrm{Pt}(111)$ are compared with results from integrating rate equations from mean-field nucleation theory using self-consistent calculations for capture numbers [88].

and attempt frequency of terrace diffusion [121-124]. The accuracy of these numbers can be further increased in analyzing cluster densities down to lower temperatures [88, 125]. For $D / F<10^{5}$, Equation 20.7 no longer holds because monomers diffuse too slowly to reach each other and create all nuclei during deposition. Therefore, monomers are stable nuclei $(i=0)$ in the sense that many of them remain monomers on the timescale of deposition. They continue to diffuse after the shutter of the evaporator has been closed. This postdeposition mobility gives rise to cluster growth and cluster nucleation in the time between deposition and imaging with STM. It is clearly visible in Figure 20.8d that this leads to a reduced slope ending with a plateau where all islands are created after deposition, and thus $n_{x}$ becomes independent of the deposition temperature [121, 126]. Postdeposition 
mobility can be accounted for in rate equations using mean-field nucleation theory. These equations have been integrated using self-consistent solutions to the capture numbers derived by Bales and Chrzan [127]. As a result, the experimental data for $\mathrm{Ag} / \mathrm{Pt}(111)$ could be fitted over a range of almost 10 orders of magnitude in $D / F$, yielding $E_{\mathrm{m}}=168 \pm 5 \mathrm{meV}$ and $v_{0}=7 \times 10^{13 \pm 0.3} \mathrm{~s}^{-1}$ [88]. Similar precision was obtained by Bott et al. [125] for Pt(111) self-diffusion.

Equation 20.6 and Equation 20.7 have been subjected to extensive experimental tests and to tests with kinetic Monte Carlo (KMC) simulations [28], all showing that for $10^{5} \leq D / F \leq 10^{9}$, these equations unambiguously relate $D / F$ to $n_{x}$ [88]. The experiments studying $n_{x}(T)$ in the irreversible growth regime $(i=1)$ yield a precision for $E_{\mathrm{m}}$ close to that of state-of-the-art FIM studies, which presented, before the VT-STM studies, our most precise experimental source of information on atomic diffusion barriers. In contrast to FIM, however, the nucleation method is not limited to highly refractive elements and therefore enables the study of monomer diffusion for a wide range of film elements on substrates as different as metals, semiconductors, thin epitaxial oxide and nitride films, and surface-adsorbed graphene. This method has been applied to measure the influence of isotropic strain on diffusion on $\mathrm{fcc}(111)$ surfaces [128]. This inspired $a b$ initio calculations revealing that the binding energy of the bridge site becomes less affected by strain than that of the threefold hollow site, leading to the observed strong effect of strain on $E_{\mathrm{m}}$, which is the difference of both energies $[112,129]$.

Also systems with extremely small diffusion barriers that were formerly inaccessible by experiment, such as $\mathrm{Al} / \mathrm{Al}(111)$ [130-133] and $\mathrm{Al} / \mathrm{Au}(111)-(\sqrt{3} \times 22)$ [28, 134], can be addressed. However, these studies have to be performed with special care. the first reason is that systems with small diffusion barriers are more sensitive to minute amounts of impurities [133]. The second reason is that there might be interactions between the diffusing atoms, as discussed in more detail later. For systems with small barriers, these interactions become comparable to the diffusion barrier and therefore they induce important deviations from Equation 20.6 and Equation 20.7 [135, 136]. The third reason is that for some systems, dimer diffusion might be activated from very low $T$ on, and its neglect leads to wrong conclusions on $E_{\mathrm{m}}$ [132]. The preexponential factor $v_{0}$ obtained from an analysis of $n_{x}$ in terms of Equation 20.7 is a good indicator whether the studied system exhibits one or more of these three features. It is often much smaller than the expected value if there are adatom interactions, either mutual or with foreign adatoms that are not considered in the analysis [133]. It remains to be seen which of the very low preexponential factors discussed in the literature are real [130].

The cluster size distributions obtained by nucleation on homogeneous substrates all fall onto common curves that depend only on the critical cluster size $i$ [137-141]. The scaling law is obtained when the size distributions are plotted as $n_{N} \times\langle N\rangle^{2} / \Theta$ versus $N /\langle N\rangle$, where $N$ and $\langle N\rangle$ are the size and its mean value, respectively, and $n_{\mathrm{N}}$ is the density of $\mathrm{N}$-sized clusters. The shape of the size distributions is a Gaussian with a half width at half maximum (HWHM) of $\sigma \sim 0.55$ in the case of $i=1$ [141]. We will discuss later several methods for significantly narrowing down the size distributions. 
20.4.3

\section{Nucleation in the Presence of Long-Range Interactions}

Nucleation gets significantly modified if the diffusing adatoms exhibit mutual interactions. It is facilitated if these interactions are attractive. Examples are the adatom binding energy differences close to steps or to islands, leading to denuded zones of adatoms down to very low temperatures [90, 91]. Repulsive interactions delay nucleation and significantly change the scaling behavior of $n_{x}$ with $D / F[136,142,143]$.

Here we address interactions that are repulsive on a relatively short range of one or two atomic distances, and oscillatory over much larger distances. Such interactions exist on surfaces having a Shockley-type surface state $[144,145]$. They go back to screening in this quasi-2D electron system (for surface states and electron screening, see Chapter 5 of Volume 2). We first discuss electron screening in 3D bulk, and then in 2D surface states, we show examples for adatom interactions mediated by them, and finally, we present very well ordered adatom superlattices that they can create.

An impurity atom in a $3 \mathrm{D}$ solid induces a variation in the potential acting on the host conduction electrons, which they screen by oscillations in their density. Friedel [146] introduced such oscillations with wave vector $2 k_{\mathrm{F}}$, where $k_{\mathrm{F}}$ is the Fermi wave vector, to calculate the conductivity of dilute metallic alloys. In addition to the pronounced effect on the relaxation time of conduction electrons, Friedel oscillations are a source of mutual interactions between impurity atoms, as the binding energy of one such atom in the solid depends on the electron density into which it is embedded, and this quantity oscillates around another impurity atom. Lau and Kohn [147] predicted such interactions to depend on distance as $\cos \left(2 k_{\mathrm{F}} r\right) / r^{5}$. We note that for isotropic Fermi surfaces, there is a single $k_{\mathrm{F}}$ value, whereas in general, one has to insert the Fermi vector pointing in the direction of the interaction $[148,149]$. The electronic interactions are oscillatory, and their $1 / r^{5}$ decay is steeper than the monotonic $1 / r^{3}$ decay of elastic interactions [150]. Therefore, elastic interactions between bulk impurities dominate the electronic ones from relatively short distances on.

This situation is quite different in two dimensions. The pair interaction energy between two impurities caused by screening in a 2D electron gas was predicted to be proportional to $\cos \left(2 k_{\mathrm{F}} r\right) / r^{2}$ [147]. This relatively slow decay implies that electronmediated interactions in two dimensions dominate elastic and dipolar ones, giving rise to interactions between impurities that oscillate between attraction and repulsion as a function of distance. The first experimental observation indicative of long-range interactions, possibly mediated by the 2D Friedel oscillations, came from equidistant bulk-segregated impurities on $\mathrm{Cu}(111)$ [151]. Clear evidence for their oscillatory nature has been provided by quantitative studies determining the interaction energy as a function of distance for $\mathrm{Cu} / \mathrm{Cu}(111)$ [144] and their electronic origin has been proved for two substrates with different electronic structure at the surface modifying the period of the oscillatory interactions [145]. 
The 2D nearly free electron gas causing these interactions is provided by the Shockley-type surface states of close-packed surfaces of noble metals. These states are located in narrow band gaps in the center of the first Brillouin zone of the (111)-projected bulk band structure [152]. The fact that their occupied bands are entirely in the bulk band gaps separates the electrons in the 2D surface state from those in the underlying bulk. Only at structural defects, such as steps or adsorbates, there is overlap of the wave functions, opening a finite transmission between the 2D and the 3D system. The fact that the surface-state band is narrow implies extremely small Fermi wave vectors, and consequently, the Friedel oscillations of the surface state have a significantly larger wavelength than those of bulk states.

STM images taken at low bias directly reflect the oscillating quantity, namely, the LDOS (local density of states) close to $E_{\mathrm{F}}$, thus enabling direct observation of the Friedel oscillations [153]. The Friedel oscillations in surface states were first observed for $\mathrm{Cu}(111)$ [154], closely followed by $\mathrm{Au}(111)$ [155]. Figure 20.9a shows the Friedel oscillations on $\mathrm{Ag}(111)$ that has a surface state with $k_{\mathrm{F} \text {,surf }}=$ $0.083 \AA^{-1}$ [156] $\left(k_{\mathrm{F}, \text { bulk }}=1.2 \AA^{-1}\right.$ [157]). There are two substitutional defects appearing as protrusions on the otherwise clean surface. They induce a smooth modulation in the apparent height of the Ag atoms, extending over the entire image. These are the surface-state Friedel oscillations [154] that are readily detectable up to more than $100 \AA$ distance in the large-scale STM image in Figure 20.9b.

The Friedel oscillations concentric around individual $\mathrm{Cu}$ atoms on $\mathrm{Cu}(111)$ are seen in Figure 20.10a. They have shorter wavelength than those on $\mathrm{Ag}(111)$ because of the larger value of $k_{\mathrm{F}}=0.21 \AA^{-1}$ [154]. This STM image is one out of a sequence recorded on the same surface spot at $13.5 \mathrm{~K}$, see the authors website (http://lns.epfl.ch) for the corresponding movie showing how the $\mathrm{Cu}$ adatoms diffuse. The atoms come quite often close to each other; however, they remain isolated rather than forming islands. This indicates the existence of short-range repulsions. For the present system, no aggregation was observed after annealing at $16.5 \mathrm{~K}$ for $20 \mathrm{~min}$. Upon annealing during a

a)

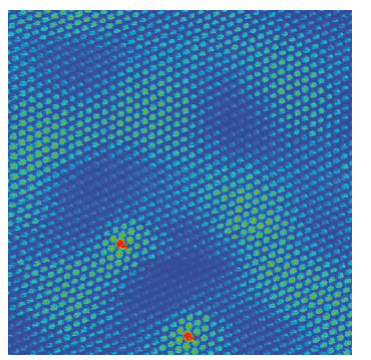

$20 \AA$ b)

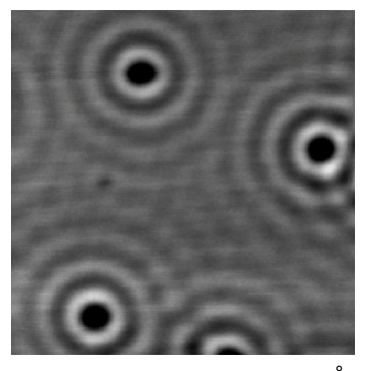

$100 \AA$

Figure 20.9 (a) Friedel oscillations around two substitutional defects on $A g(111)\left(V_{t}=-5 m V\right.$, $I_{\mathrm{t}}=8 \mathrm{nA}, T=9 \mathrm{~K}$. (b) Large scale image showing the long-range oscillations with $\lambda=\pi / k_{\mathrm{F}}=38 \AA$ around four point defects on $\mathrm{Ag}(111)\left(V_{\mathrm{t}}=24 \mathrm{mV}, \mathrm{I}_{\mathrm{t}}=0.5 \mathrm{nA}, T=9 \mathrm{~K}\right.$. (From Ref. [145].) 
a)

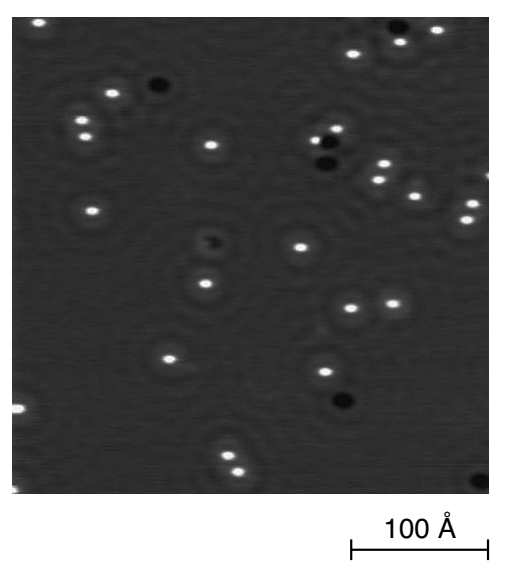

b)
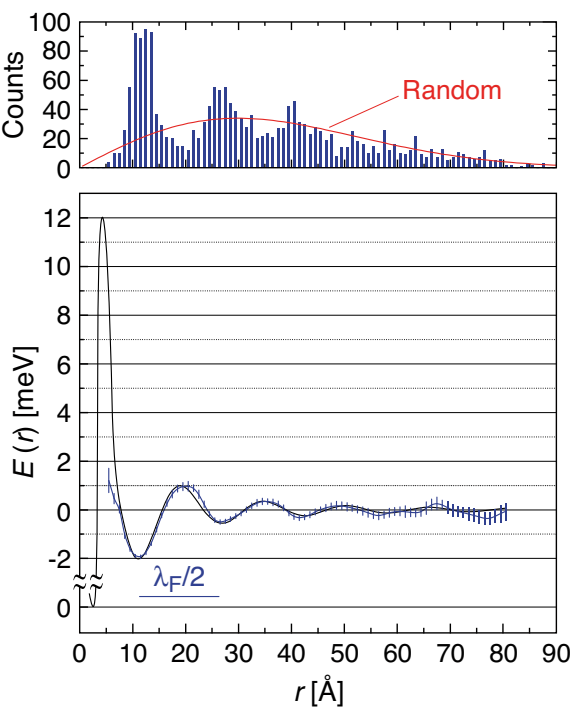

Figure 20.10 (a) Surface-state Friedel oscillations around $\mathrm{Cu}$ adatoms (bright) and substitutional impurity atoms (black) on $\mathrm{Cu}(111)\left(\Theta=1.4 \times 10^{-3} \mathrm{ML}, T=13.5 \mathrm{~K}\right)$ [145]. (b) Pair distance histogram showing a significant deviation from a random distribution (red line) and the derived pair interaction energy for large distances [145]. The thin black line shows for large distances a fit with Equation 20.8. For the repulsion at short distances see text. The dimer bond energy is taken from $a b$ initio calculations [161].

comparable time at $22 \mathrm{~K}$, almost all the monomers formed islands. From these observations, the short-range repulsion has been estimated to $12 \pm 2 \mathrm{meV}$ [136]. This energy can only partly be caused by the surface-state Friedel oscillations. Its main origins are dipole-dipole, elastic, or bulk-electron-mediated interactions. Such short-range interactions have been studied by means of FIM $[95,158]$ and STM $[140,159]$. We note here that the short-range repulsion is mandatory for the observation of the long-range interactions because it stabilizes the adatom gas against nucleation.

Figure 20.10a shows a few adatom pairs with the same distance. Analysis of many such images in terms of pair distance abundances revealed significant deviations from a random distribution, as seen in the histogram in Figure 20.10b. These deviations can be translated with the Boltzmann statistics into pair interaction energies. The obtained pair potential oscillates with a wave vector of $2 k_{\mathrm{F}}$ and shows an asymptotic $1 / r^{2}$ decay (Figure 20.10b). Both findings are in perfect agreement with theory [160].

The experimentally determined potential is of the form

$$
E(r)=-A E_{0}\left(\frac{2 \sin (\delta)}{\pi}\right)^{2} \frac{\sin (2 q r+2 \delta)}{(q r)^{2}+(q c)^{2}}
$$

where $E_{0}$ is the onset energy of the surface state and $c$ is a fit parameter accounting for the attenuation of the first minimum with respect to the $1 / r^{2}$ decay. The 
position and amplitude of the first minimum determine the scattering phase $\delta=(0.50 \pm 0.07) \pi$ and amplitude $A=0.13 \pm 0.01$. The phase is in good agreement with a black-dot scatterer. The best fit is obtained with $c=10 \AA$, meaning that the first minimum is slightly attenuated with respect to $1 / r^{2}$. This is likely due to the superposition of the surface-state-mediated $E(r)$ with repulsive elastic or 3D electronic interactions at short distances. Ab initio calculations of interaction energies for $\mathrm{Co} / \mathrm{Cu}(111)$ [162] yield an attenuated first minimum and thereby are in much better agreement with experiment [145] than the scatter model [160]. Independent of this attenuation, the first minimum of $E(r)$ is clearly more attractive than the second one, in contrast with the curve reported in Ref. [144], where presumably many-body interactions were not eliminated in the analysis. The wave vector $q=(0.20 \pm 0.01) \AA^{-1}$ is in good agreement with the band structure of the $\mathrm{Cu}(111)$ surface state (see earlier discussion). This is also true for interactions with Co atoms on $\operatorname{Ag}(111)$, where $q=(0.10 \pm 0.02) \AA^{-1}, \delta=(0.33 \pm 0.02) \pi$, $A=0.29 \pm 0.01$, and $c=3 \AA[145]$.

Early attempts to use these interactions for the formation of atomic superlattices failed $[144,145]$. The breakthrough came for the system $\mathrm{Ce} / \operatorname{Ag}(111)$, where Figure 20.11a,b reveal very well ordered hexagonal superlattices with a lattice constant of $32 \AA$ [163]. This dilute atomic superlattice is most nicely ordered at 3.9 $\mathrm{K}$, whereas the atoms start to diffuse around their ideal positions at $4.8 \mathrm{~K}$. At that point, the system corresponds to a $2 \mathrm{D}$ dilute liquid. At $10 \mathrm{~K}$ already, the lattice is destroyed by irreversible nucleation of Ce islands [163].

The confinement of surface-state electrons by static scatterers results in stationary LDOS patterns that influence the adsorbate binding energy and can thereby impose preferred adatom adsorption sites. In 1D structures formed by substrate steps and by strings of adsorbed atoms or molecules, this leads to 1D confinement of individual adsorbed atoms [164-167]. The surface-state LDOS patterns formed in a network of hexagonal molecular cavities have been demonstrated to influence the binding sites of adsorbed CO molecules [168]. Single Fe atoms have been found at preferred distances inside hexagonal cavities of the metal-organic honeycomb network formed by $\left(\mathrm{NC}-\mathrm{Ph}_{5}-\mathrm{CN}\right)_{3} \mathrm{Cu}_{2} / \mathrm{Cu}(111)$ lattices [169]. The cavity occupancy is given by the statistics of deposition, that is, by a binomial distribution [170].

a)

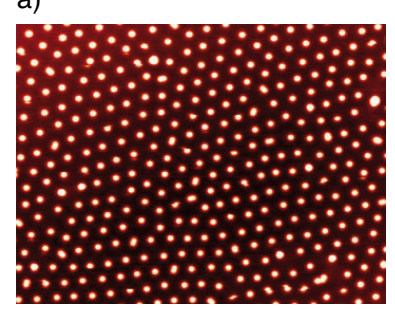

$10 \AA$ b)

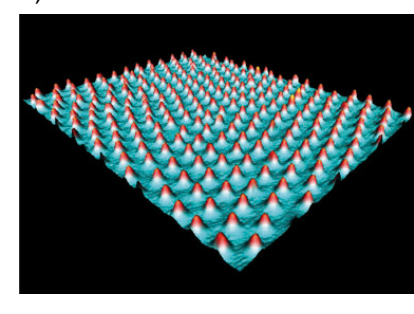

Figure 20.11 (a) Top view and (b) 3D view of an STM image showing a well-ordered hexagonal Ce superlattice formed on a $\operatorname{Ag}(111)$ surface $\left(\Theta=8 \times 10^{-3} \mathrm{ML}, T=3.9 \mathrm{~K}\right)$. (Kindly provided by W. D. Schneider [163].) 
Upon annealing, the individual Fe atoms in each cavity condense to a single island centered in the respective cavity, thus creating well-ordered Fe cluster superlattices with the lattice constant of $5 \mathrm{~nm}$ of the metal-organic honeycomb network [169].

20.4.4

Heterogeneous Nucleation

The above-mentioned example with equidistant static electron scatterers that cause binding energy differences of the adsorbates through 2D Friedel's oscillations marks the transition from homogeneous nucleation via adsorbate interactions to heterogeneous nucleation. Heterogeneous nucleation takes place when the mean-free path of the diffusing film atoms or molecules is comparable or larger than the mean distance between point or line defects. These defects may either act as nucleation sites or have a repulsive interaction with the adsorbates. The most prominent case for attractive line defects is the atomic steps acting as sinks for diffusing adatoms. In the resulting step-flow growth, films grow without nucleation at all, but solely by attachment of adatoms into ascending and possibly also descending steps.

An example of attractive point defects is metal nucleation on $\mathrm{MgO}(100)$ [171, 172]. A first indication that point defects play an important role for that system comes from the cluster density critically depending on the surface preparation. Cleavage of bulk $\mathrm{MgO}$ in air, and subsequent deposition under UHV conditions, yields a 10 times higher saturation island density than UHV cleavage and in situ deposition [173]. On the other hand, cleavage in Ar atmosphere with subsequent annealing in oxygen $\left(750 \mathrm{~K}, p_{\mathrm{O}_{2}}=1 \times 10^{-4}\right.$ mbar) gives the same densities as UHV cleavage [174]. This indicates that oxygen vacancies are involved, as their density is reduced by annealing in oxygen.

Quantitative information on the nucleation kinetics of Pd on $\mathrm{MgO}(100)$ has been obtained by measuring $n_{x}(T)$ with VT-AFM [174]. The AFM images reproduced in Figure 20.12 show that nucleation takes place predominantly at terraces and only occasionally at steps. The Arrhenius plot of the saturation island density shows a large plateau between 200 and $600 \mathrm{~K}$, indicating heterogeneous nucleation at defects with large trapping energies. The trapping defects must thus be located in midst of substrate terraces. There are several types of oxygen vacancies on $\mathrm{MgO}(100)$. According to $a b$ initio calculations, the so-called neutral $F_{\mathrm{s}}$ center is the most probable candidate for attractive point defects in this case $[172,175]$.

Fitting the experimental data with mean-field nucleation theory including trapping defects yields the solid line in Figure 20.12. The diffusion energy on the defect-free $\mathrm{MgO}(100)$ terraces must be $E_{\mathrm{m}} \leq 0.2 \mathrm{eV}$, as all the Pd atoms reach the defect sites down to $T=200 \mathrm{~K}$. The length of the plateau toward higher $T$ defines the minimum trapping energy, $E_{\mathrm{t}} \geq 1.2 \mathrm{eV}$. This bound compares reasonably well with the theoretical value of $E_{\mathrm{t}}=1.55 \mathrm{eV}$ [175]. The knee at $600 \mathrm{~K}$ is best reproduced by a transition from $i=1$ to $i=3$, that is, traps remain populated by Pd atoms, but four atoms are needed for a stable cluster at this temperature. The deduced lateral bond energy between $\mathrm{Pd}$ atoms of $E_{\mathrm{b}}=1.2 \mathrm{eV}$ lies slightly below the theoretical 


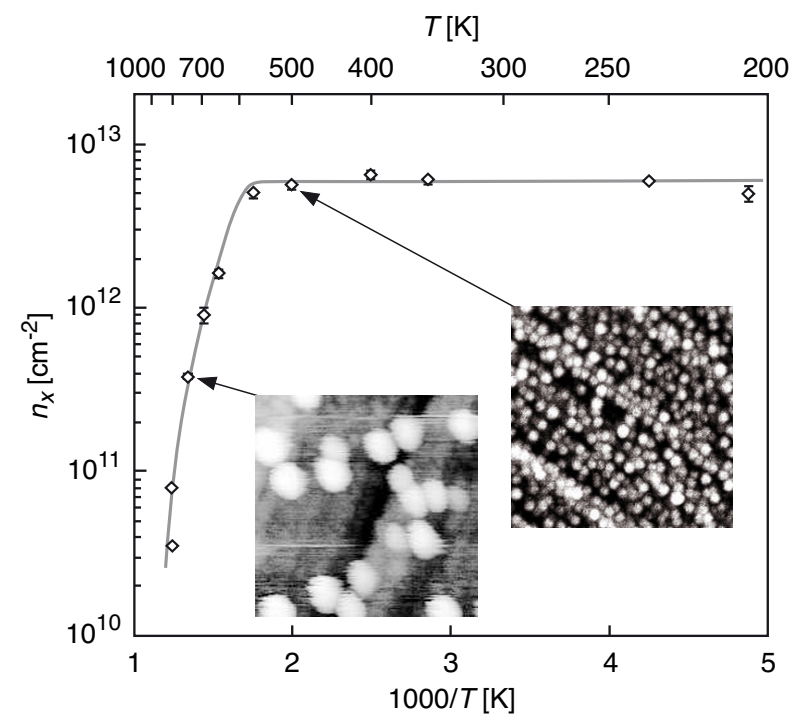

Figure 20.12 Arrhenius plot of saturation island densities for Pd deposits on Ar-cleaved $\mathrm{MgO}(100)$ obtained with noncontact AFM (size of images $1000 \times 1000 \AA$ ) [174]. The solid line is a mean-field rate equation model accounting for defect trapping $\left(\Theta=0.1 \mathrm{ML}, F=2.7 \times 10^{-4} \mathrm{ML}\right.$ $\mathrm{s}^{-1}$ ). See text for model parameters.

gas-phase value, which is reasonable on a weakly bonding substrate. Incomplete condensation starts at $750 \mathrm{~K}$, as evidenced by the decrease in Pd coverage at constant exposure deduced from the Auger electron spectroscopic (AES) measurements, and by the final increase in the slope of $\log \left(n_{x}\right)$ versus $1 / T$, very well reproduced in the model for an adsorption energy of $E_{\mathrm{a}}=1.2 \mathrm{eV}$. This parameter set has been derived for the model system $\mathrm{Pd} / \mathrm{MgO}(100)$ under the assumption of $i=1$. Note that a more recent DFT study finds that Pd dimers are unstable on terraces and that $F_{\text {s }}$ centers are strong traps for monomers but stabilize dimers only weakly [172]. Therefore, these energy values might have to be attributed to somewhat larger species than that in the presented rate equation model. Note that thin $\mathrm{MgO}(100)$ films grown on metal surfaces lead to different charge states of adsorbed metal atoms than thick films or cleaved bulk samples [176]. For the structure and catalytic activity of small metal clusters on $\mathrm{MgO}(100)$, see Refs [177-181].

A particularly interesting case of heterogeneous nucleation is when template surfaces with regularly spaced point defects are used for the self-assembly of wellordered 2D island or 3D cluster superlattices. Note that lattices of partial surface dislocations that act as repulsive line defects are similar to a lattice of attractive pointlike defects [170]. Figure 20.13 shows three examples of heterogeneous nucleation on substrates with regularly spaced attractive defects.

The moiré structure of CVD grown graphene on $\operatorname{Ir}(111)$ is shown in Figure 20.13a. Two substrate steps separate three terraces that are entirely covered by a moiré pattern with an incommensurate unit cell that spans $(9.32 \pm 0.15 \times 9.32 \pm 0.15)$ substrate unit cells [182]. Roughly speaking, in each of the two close-packed 
a)

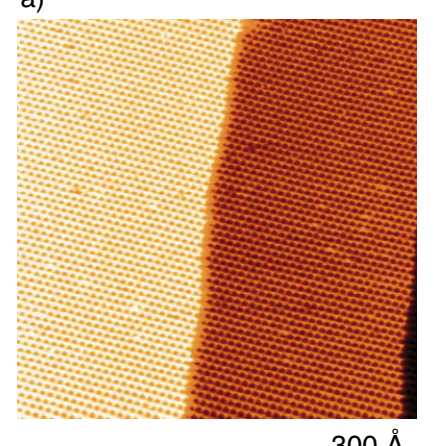

$300 \AA$

c)
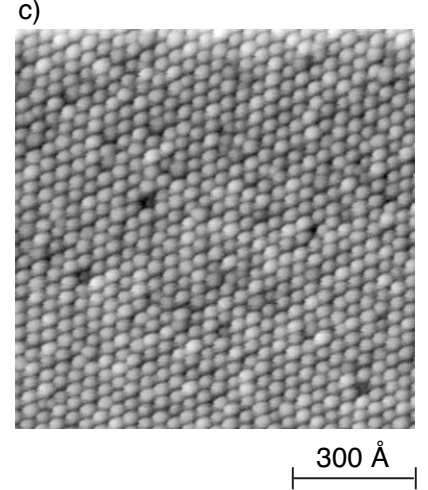

b)

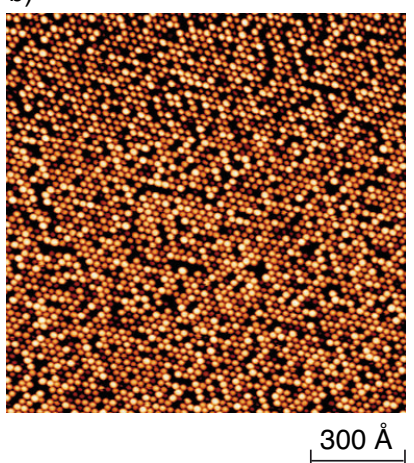

d)

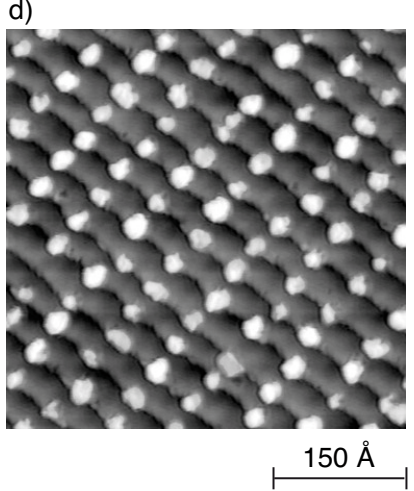

Figure 20.13 (a) STM image of the moiré of graphene on $\operatorname{Ir}(111)\left(T_{\mathrm{CVD}}=1300 \mathrm{~K}\right)$. (b) Ir cluster superlattice grown on this template $\left(\Theta=0.15 \mathrm{ML}, T_{\text {dep }}=375 \mathrm{~K}\right)$. (From Ref. [43].) (c) Pd cluster superlattice grown on $2 \mathrm{ML} \mathrm{Al}_{2} \mathrm{O}_{3} / \mathrm{Ni}_{3} \mathrm{Al}(111)-(\sqrt{67} \times \sqrt{67}) \mathrm{R} 12.2^{\circ}\left(\Theta=0.36 \mathrm{ML}, T_{\text {dep }}=300 \mathrm{~K}\right.$, $F=0.3 \mathrm{ML} \mathrm{s}^{-1}$ ). (From Ref. [183].) (d) STM image showing Co bilayer islands self-assembled on $\operatorname{Au}(788)\left(\Theta=0.35 \mathrm{ML}, T_{\text {dep }}=130 \mathrm{~K}, T_{\text {ann }}=300 \mathrm{~K}\right)$. (From Ref. [184].)

substrate directions, there are 10 graphene unit cells on 9 Ir atoms and the beating of the two lattice constants causes the moiré pattern.

Upon deposition of Ir atoms at $375 \mathrm{~K}$ onto this surface, one Ir island nucleates per moiré unit cell [185]. As seen in Figure 20.13b, this gives rise to a well-ordered hexagonal lattice of Ir clusters [43]. For the coverage used in this experiment, the mean cluster size is 13 atoms. Apart from the interest of such clusters in catalysis and for seeding of magnetic materials, these clusters act as point scatterers for the electrons in the underlying graphene and lead to a band gap of $0.34 \mathrm{eV}$, while fully preserving the graphene electron group velocities, a necessary condition for the high charge carrier mobilities required in applications [34]. A similar effect has been obtained by $\mathrm{H}$ adsorption on specific sites of the moiré pattern [186]. However, the approach with Ir clusters is robust to higher temperatures. The gap can further be increased to $0.74 \mathrm{eV}$ by the co-adsorption of $\mathrm{Na}$, still largely preserving the high group velocities [35]. 
The $\mathrm{Ni}_{3} \mathrm{Al}(111)$ surface forms a self-limiting, $2 \mathrm{ML}$ thick oxide with a $(\sqrt{67} \times$ $\sqrt{67}$ R12.2 unit cell that has corner holes going all the way down to the metal substrate [187]. Pd atoms fill these holes building stable traps on which further $\mathrm{Pd}$ atoms or other elements can be condensed [183,187]. Figure 20.13c shows the resulting perfectly ordered superlattice of 3D Pd clusters [183].

All templates have in common that their superstructure may have phase jumps between adjacent terraces. These are evidently reproduced in the cluster superlattices. This is circumvented by vicinal surfaces. Figure 20.13d shows a $\mathrm{Au}(788)$ surface, where the steps are the energetically favorable $\{111\}$-microfacets, which we will call B steps in the following section. Because of elastic repulsions, these steps arrange equidistantly over the entire crystal [188]. The (111)-oriented terraces exhibit a reconstruction similar to the $(\sqrt{3} \times 22)$ reconstruction of $\mathrm{Au}(111)$ [189]. However, on the vicinal surface, it has a slightly lager period of $7.2 \mathrm{~nm}$, the domain walls run perpendicular to the step edges, and are aligned from terrace to terrace. Nucleation of Co takes place at the crossing of the domain walls and the steps $[188,190]$. As Figure 20.13d shows, deposition at low $T$ with subsequent annealing creates a well-ordered superlattice of double layer high Co islands with uniform size (HWHM $=20 \%)$. As the reconstruction pattern, this lattice is phase coherent over the entire crystal. While the island distance along the steps is given by the reconstruction period, the perpendicular distance can be chosen by the miscut of the vicinal crystal surface toward (111). Au (788) has step distances of $3.8 \mathrm{~nm}$, while $\mathrm{Au}(11,12,12)$ is closer to $(111)$ and has an average step distance of $5.9 \mathrm{~nm}$. In addition to the shown example of Co clusters [190, 191], Fe makes ordered islands on these surfaces [192]. For the fascinating magnetic properties of these ensembles of magnetically noninteracting, uniaxially out-of-plane magnetized islands of well-defined sizes, we refer to Refs [190, 193].

\section{5}

\section{Island Shapes}

The similarity of patterns formed in nonequilibrium growth processes in physics, chemistry, and biology is conspicuous, and many attempts have been made to discover common mechanisms underlying their formation [194, 195]. The most prominent examples are snowflakes. The correlation of their shape with meteorological crystal growth conditions provides valuable information on the pressure, humidity, and temperature in the upper atmosphere [196]. The many growth patterns in nature commonly evolve from nonequilibrium growth at an interface with material transport via diffusion being the rate-limiting process. These conditions exactly govern aggregation of clusters at single-crystal surfaces held at low temperatures. Aggregation of submonolayer coverages of metal atoms is often 2D and therefore easier to understand than many more complex growth patterns. Nevertheless, it bears enough complexity that knowledge gained in 2D may be transferred to the understanding of more complicated 3D patterns appearing in nature. This makes low-temperature metal aggregation an ideal model system 
for tracing back mechanisms by which single-diffusion events of atoms along a growing interface translate into an overall pattern. STM opened access to the structure of monolayer-high 2D aggregates formed by atoms at surfaces [197], and its extension to variable low temperatures enabled the investigation of the kinetics underlying the formation of such patterns down to temperatures where irreversible growth occurs [198-201]. The knowledge gained from microscopic studies in turn provides control over the shape of MBE-grown surface-supported clusters. We discuss ramified islands with fractal dimension and various compact island shapes formed on isotropic single-crystal metal surfaces.

20.5.1

Fractals

The simplest scenario studied in theory is DLA [97, 98] in 2D, where atoms laterally arriving at the cluster edge come to rest at their point of contact. This leads to the formation of monoatomic wide branches that spread out into random directions. Also in the case of slow edge diffusion, with barrier $E_{\mathrm{e}}$ in Figure 20.7, ramification occurs and branches form. Low-edge mobility leads to the so-called tip or the Saffman-Taylor instability [202]. Every protrusion at the edge, resulting from statistical fluctuations, captures more adatoms from the terrace diffusion field than straight or concave step sections. If these atoms cannot be transported away sufficiently fast by edge diffusion, the protrusions grow out as a branch with a width related to the step-edge diffusion barrier [203-206]. With increasing length, the branch tip becomes exposed to an increased solid angle of aggregating adatoms causing ramification into more branches. The continued ramification leads to the self-similarity of the growth patterns, and a fractal Hausdorff [195] dimension of close to 1.7, that is, the cluster area increases as $A=r^{1.7}$, with $r$ being the radius. Note that the ice crystal shown in Figure 20.1a also has self-similarity and a fractal dimension, which is evidently higher because that object has grown in 3D space.

The theoretical DLA clusters have so far never been observed in the growth on single-crystal surfaces. Depending on the surface symmetry, there are different reasons why they are not realized. On square lattices, there are only onefoldcoordinated step sites and edge diffusion has a barrier comparable to that of terrace diffusion [207-209]. As soon as terrace diffusion gets thermally activated, diffusion along the cluster edge is activated, too. This generally leads to compact square islands at any deposition temperature [124, 126, 210-214]. An exception is the formation of noncompact islands observed for $\mathrm{Cu} / \mathrm{Ni}(100)$, which has been attributed to strain-induced increase in the step length [215].

On hexagonally close-packed (hcp) urfaces, there are corner sites with onefold lateral coordination and sites at straight $\langle 1 \overline{1} 0\rangle$-oriented edges with twofold coordination. Atoms bound to the twofold edge sites have high $E_{\mathrm{e}}$ values and thus low mobility, and this generates the branched islands seen in Figure 20.8a-c and Figure 20.14. In contrast to DLA clusters, they have trigonal symmetry and their branches are at least two to three atoms wide. Nevertheless, their fractal dimension is close to the DLA value of 1.7. These islands have been called dendrites by analogy 
a)

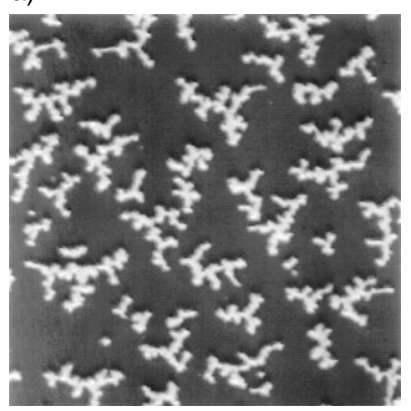

$100 \AA$ b)

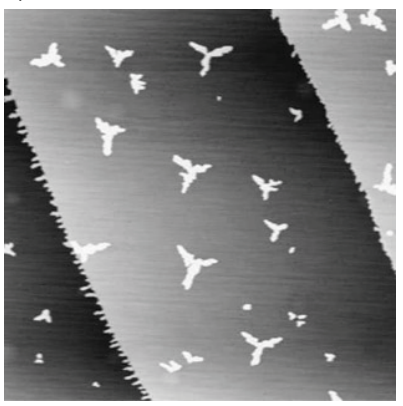

$200 \AA$

Figure 20.14 Dendritic islands formed for low-temperature metal deposition onto close-packed metal substrates. (a) $\mathrm{Pt}(111)$ homoepitaxy at $180 \mathrm{~K}(\Theta=0.094 \mathrm{ML})$ [216]. (b) Pt heteroepitaxy on $\mathrm{Ru}(0001)$ at $300 \mathrm{~K}(\Theta=0.1 \mathrm{ML})[217]$.

with other patterns in nature revealing preferred growth directions [199]. The three preferred growth directions lead to $Y$-shapes for small cluster sizes (Figure 20.8a,b and Figure 20.14b). Owing to the absence of further branching, these Y's have a Hausdorff dimension of 1.0. Larger dendrites have a triangular envelope, as evident from the inspection of Figure 20.8c and Figure 20.15c. Note that in the case of $\mathrm{Pt} / \mathrm{Ru}(0001)$ shown in Figure 20.14b, the substrate has hcp AB-stacking leading to a rotation of the dendrites by $60^{\circ}$ from terrace to terrace.

The reason for the slightly larger than monoatomic branch width in dendrites is connected with the relaxation of atoms adsorbed on laterally onefold-coordinated corner sites to twofold step sites. This relaxation is asymmetric being one of the reasons for the preferred growth directions [201, 216]. The diffusion asymmetry from corner to edge sites can be inferred from the ball model in Figure 20.15a. It shows that any pseudomorphic monolayer-high island placed on a hexagonal substrate is bound by two crystallographically different microfacets. The closepacked $\{111\}$-faceted B step has already been introduced. Turning by $60^{\circ}$ leads to the more open $\{100\}$-faceted so-called A step. Diffusion from the corner site labeled $\mathbf{C}$ to an A step involves an hcp site at just the right distance from the corner, whereas diffusion to the $\mathbf{B}$ step has either an on-top site or the hcp site very close to the island as transition state. From these geometric considerations, one would generally expect that corner to A-step diffusion has a lower barrier than diffusion from a corner to a B step. This picture is confirmed by effective medium theory (EMT) [207, 218] calculations for a number of metal/metal combinations for which dendritic growth perpendicular to A steps is observed [28]. Figure 20.15b shows the case of $\mathrm{Ag} / \mathrm{Pt}(111)$. Diffusion from a corner site to an A step has a barrier as low as $E_{\mathrm{m}}$, whereas diffusion to a $\mathbf{B}$ step costs five times as much activation energy. KMC simulations have demonstrated that the asymmetric corner relaxation leads to a preferred population of A steps over B steps and thus to the three preferred growth directions explaining the $Y$-shapes of small, and the triangular envelope of large islands, as evident from comparing Figure 20.15c and d. 
a)

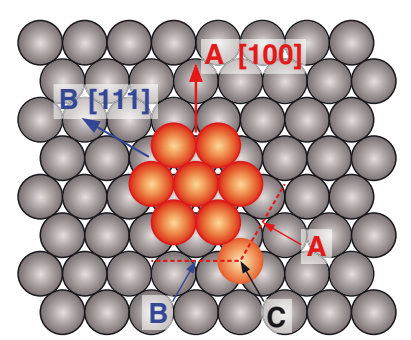

c)

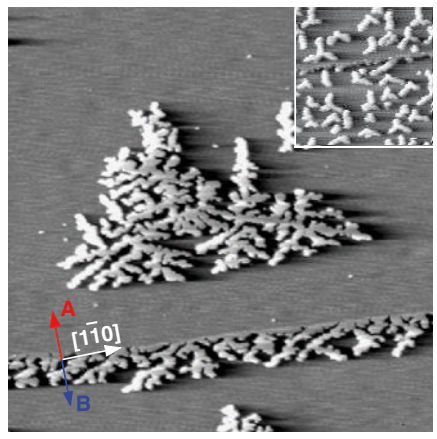

b)

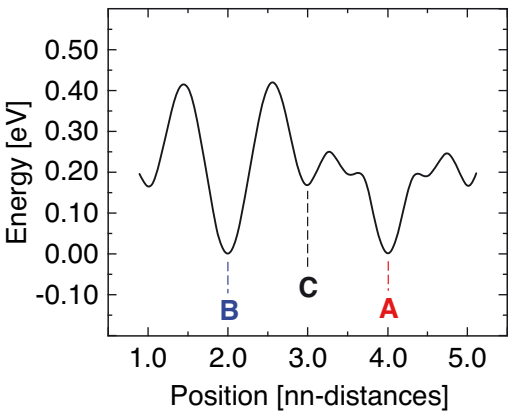

d)

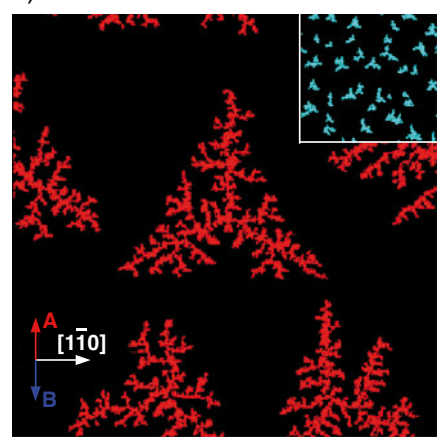

$200 \AA$

Figure $\mathbf{2 0 . 1 5}$ (a) Ball model of a heptamer on a hexagonal lattice revealing the existence of $\mathbf{A}$ and $\mathbf{B}$ steps. (b) The EMT calculation for $\mathrm{Ag} / \mathrm{Pt}(111)$ shows that this geometric difference implies different corner diffusion barriers. (c) Experiment for $\mathrm{Ag} / \mathrm{Pt}(111)$ at $130 \mathrm{~K}$ (main figure) and $80 \mathrm{~K}$ (inset). (d) The KMC simulations identify the difference in corner diffusion as the origin of dendritic growth; they reproduce the dendrites found in experiment at both growth temperatures (scale bar common to (c) and (d)) [201].

For some systems, such as $\mathrm{Cu}$ and $\mathrm{Pt} / \mathrm{Pt}(111)$, however, EMT calculations show the corner-to-edge diffusion asymmetry to be the other way around [28]. For $\mathrm{Pt} / \mathrm{Pt}(111)$, this result is confirmed by ab initio calculations [219]. Pt reveals a large tensile stress leading to a strong inward relaxation at the island edge. This can facilitate diffusion toward $\mathbf{B}$ steps, as it renders the hcp site located between corner and $\mathbf{B}$ step more attractive as a transition state. Despite this inversion of the corner to A- and B-step diffusion asymmetry, $\mathrm{Pt} / \mathrm{Pt}(111)$ shows dendritic growth with the same preferred growth direction as $\mathrm{Ag} / \mathrm{Pt}(111)$ and $\mathrm{Ag} / \mathrm{Ag}(111)$, that is, perpendicular to A steps (Figure 20.14a).

Apart from the aspect of diffusion along the island edge treated so far it was pointed out that the different diffusion paths of atoms from the terrace toward the two kinds of steps give rise to a second asymmetry favoring population of $\mathbf{A}$ steps [201]. Considering the different diffusion paths from second-neighbor fcc sites via hcp sites toward a heptamer one readily finds that there is a strong statistical preference of $3 / 6$ versus $1 / 6$ for attachment to A versus B steps, whereas corners 
are visited with a probability of only $2 / 6$ [28]. The attachment asymmetry is more general than corner diffusion asymmetry. Both effects have to be considered in conjunction to understand and predict the cluster morphology. If corner diffusion is faster toward B steps, it could counterbalance attachment and random growth may result. For systems with faster diffusion toward A steps, both effects push toward exclusive population of A steps resulting in dendritic growth perpendicular to these steps. For $\mathrm{Pt} / \mathrm{Pt}(111)$, there is a slightly smaller barrier for diffusion from corners to B steps. This anisotropy is too weak to fully counterbalance the preferred attachment to A steps, leading to dendrites that still have a growth preference perpendicular to these steps. In agreement with this preference being weaker, the trigonal symmetry is less pronounced for that system (Figure 20.14a). Looking at these rates for attachment to the two types of steps, it is clear that corner diffusion can at most fully counterbalance this asymmetry leading to random growth directions; however, branches growing preferentially perpendicular to $\mathbf{B}$ steps are not expected and to far also not observed. The growth of dendrites with trigonal symmetry on hcp surfaces is therefore well understood. This growth morphology is generally expected for islands grown on such surfaces at low enough temperatures to assure irreversible aggregation $(i=1)$.

Dendritic islands undergo several mutations upon increasing the temperature and/or reducing the flux, that is, when going to $i>1$ conditions. For some systems, the branches begin to grow into random directions, lifting the trigonal symmetry of the low-temperature dendrites [199]. In addition, the branches become wider. The shape transition from dendritic to random isotropic growth patterns is not yet fully understood $[197,206,220]$. Also a quantitative understanding of the relationship between the degree of edge diffusion and the branch width is presently lacking. We briefly review the present understanding of isotropic fractal islands. Similar to the dendrites, these patterns reveal the fractal dimension of classical DLA aggregates [197].

Examples of such fractal islands with isotropic growth directions are shown in Figure 20.16. The $\mathrm{Au}$ islands formed at room temperature on $\mathrm{Ru}(0001)$ have on average 60 atom wide branches, and the $\mathrm{Ag}$ aggregate grown at $220 \mathrm{~K}$ on $\mathrm{Pt}(111)$ has a mean branch width of $20 \pm 2$ atoms [206]. In both cases, the branches spread out and meander into random directions. Formation of partial surface dislocations between fcc and hcp stacking has been suggested to explain the loss of directional growth [28]. This was motivated by the fact that both systems shown in Figure 20.16 reveal considerable misfit, possibly leading to such dislocations or domain walls where strain is relieved. Each stacking variation from hcp to fcc alters the orientation of $\mathbf{A}$ and $\mathbf{B}$ steps and thus the branches' preferred growth directions. Regular stacking faults could thus explain the observed random isotropic growth. Partial surface dislocations have been observed for $\mathrm{Ag} / \mathrm{Pt}(111)$ upon a critical island size [221], which is however beyond the branch width for which the onset of random growth is observed. It was thus proposed that surface partials were generated by kinetics, and a KMC model showed that the transition from dendritic to random fractal clusters can be generated that way [28]. 
a)

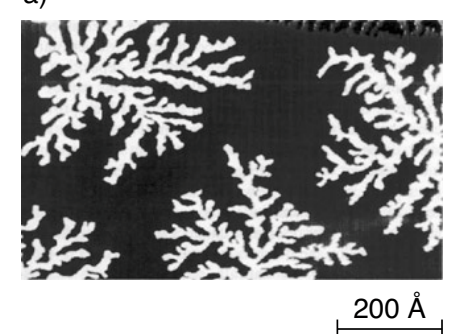

b)

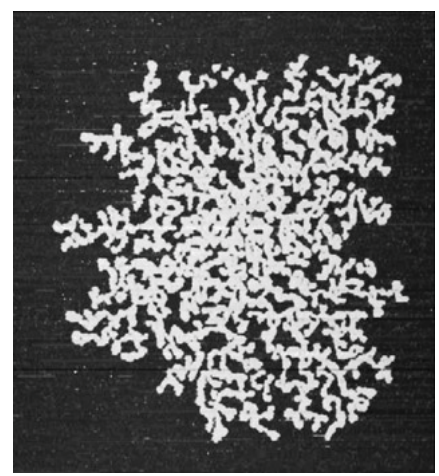

$600 \AA$

Figure 20.16 Examples of fractals with random growth directions. (a) STM image of Au islands grown on $\mathrm{Ru}(0001)$ ( $\left.T_{\text {dep }}=300 \mathrm{~K}, F=3.3 \times 10^{-3} \mathrm{ML} \mathrm{s}^{-1}, \Theta=0.30 \mathrm{ML}\right)$ [197]. The aggregate's fractal dimension is the DLA value of 1.72. (b) STM image of a $\mathrm{Ag}$ fractal grown on $\mathrm{Pt}(111)$ at $220 \mathrm{~K}$ similarly showing wide branches that frequently alter their growth direction $\left(F=1.1 \times 10^{-3}\right.$ $\mathrm{ML} \mathrm{s}^{-1}, \Theta=0.12 \mathrm{ML}$ ) [222].

The second possible origin for the loss of directional growth is the detachment from onefold-coordinated corner sites, as $i>1$. The arguments of corner diffusion and diffusion paths for attachment to the different step types given earlier have to be reexamined under reversible growth. This might well lead to the finding that frequent detachment of onefold atoms leads to the observed random growth directions.

The branch width was related to edge diffusion, both by analytic models [203, 205, 214] and through KMC simulations [204]. The basic idea underlying these studies is that the lateral impingement rate $I$ competes with the rate for an adatom to scan the edge of a compact seed particle. The seed particle stays compact until its edges reach a critical width $w$ where both rates become comparable. At that point, nucleation of protrusions at the edge can no longer be flattened out and the aggregate becomes unstable upon ramification through the Mullins-Sekerka instability introduced earlier. This instability argument defines the critical cluster size for ramification and equally the mean branch width taken on by the cluster after ramification.

However, the models presented in the literature disagree on the exact dependence of $w$ on $D_{\mathrm{e}} / I$, where $D_{\mathrm{e}}$ is the $1 \mathrm{D}$ diffusion rate at the edge and the impingement rate is given by $I=F / n_{x}$, once $n_{x}$ becomes saturated. Therefore, these models give contradicting results for the attempt frequencies and barriers for edge diffusion when experimental data of $w(T)$ (available for $\mathrm{Ag} / \mathrm{Ag}(111)$ and $\mathrm{Ag} / \mathrm{Pt}(111)$ [206]) are analyzed. Realistic models have to incorporate a set of parameters where diffusion barriers are attributed to step atoms depending on their coordination and the step orientation. Evidently, this leads to the well-known problem of models invoking too many parameters to allow a sensible comparison with experiment. This problem is encountered also in papers dealing with cluster diffusion, a similarly complex 
issue [223]. Ways out are either to simplify the model or to use additional input from calculations to ascribe reasonable values to the barriers. The first attempt has been made by disregarding the difference between $\mathbf{A}$ and $\mathbf{B}$ steps with a view to extracting an effective $E_{\mathrm{e}}$ value and its attempt frequency for diffusion between twofold-coordinated sites along straight steps [28]. The result $\left(E_{\mathrm{e}}=370\right.$ $\mathrm{meV}$ and $v_{0}=1 \times 10^{13} \mathrm{~Hz}$ ) is more realistic than the values obtained from the former analysis [206]. Ab initio calculations have become feasible for relatively large system sizes [135, 219, 224-226], thus allowing the calculation of the various energy barriers for adatom displacement along cluster edges and thereby providing valuable input to a more detailed understanding of how the islands in Figure 20.16 form. Random fractal islands with wide branches will certainly be of ongoing interest until we can answer the open question of how they evolve from dendrites and how their branch width is linked to the edge diffusion barrier.

20.5.2

\section{Compact Adatom and Vacancy Islands}

With increasing deposition temperature the islands on hexagonal substrates become compact triangles. Figure 20.17 shows the evolution of island shapes with deposition temperature for the case of $\mathrm{Pt}(111)$ homoepitaxy [29]. The dendrites formed at 200 and $300 \mathrm{~K}$ become triangles with straight B steps at $400 \mathrm{~K}$. They have the same orientation as the weakly triangular envelope of the dendrites, where the branches grow preferentially perpendicular to A steps. The islands remain triangles and maintain their orientation all the way up to $600 \mathrm{~K}$, until at $700 \mathrm{~K} \mathrm{a}$ quasi-hexagon forms, not shown [29]. Note that the triangles at 500 and $600 \mathrm{~K}$ are less perfect. They show a concavity in the middle of the otherwise relatively straight B steps. This has been explained by the fact that these are remains of the fjords in the branched low- $T$ island shape [29]. Another effect is certainly that the island tips capture more atoms than the straight steps, due to the Mullins-Sekerka instability discussed earlier, and as they get larger at higher temperature, it might be more difficult to transport these atoms from the tip regions to the center of the step. Further

a)

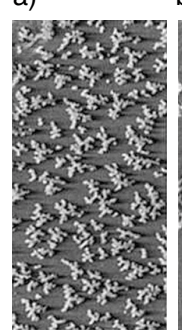

b)

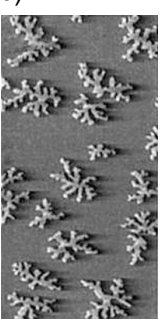

c)

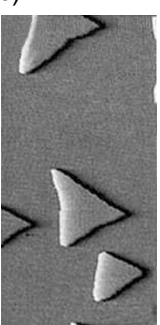

d)

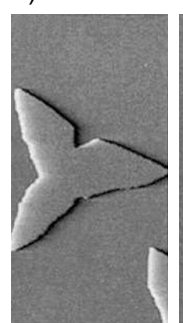

e)

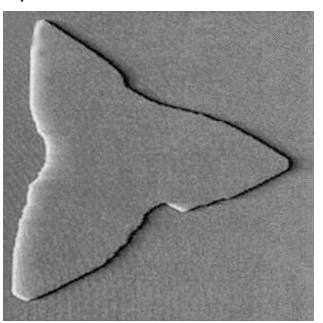

$500 \AA$

Figure 20.17 Deposition-temperature-dependent island morphology for $\mathrm{Pt}(111)$ homoexpitaxy $\left(\Theta=0.15 \mathrm{ML}, F=7 \times 10^{-3} \mathrm{ML} \mathrm{s}^{-1}, p_{\mathrm{CO}} \leq 5 \times 10^{-12} \mathrm{mbar}\right) . T_{\text {dep }}=$ (a) 200 , (b) 300 , (c) 400 , (d) 500, and (e) $600 \mathrm{~K}$. (Kindly provided by T. Michely.) 
examples for systems with triangular adatom islands with preferred orientation are $\mathrm{Co} / \mathrm{Ru}(0001)$ [220, 227, 228], Co/Pt(111) [229], and $\mathrm{Fe} / \mathrm{Au}(111)$ [230, 231].

We note that the shape sequence for $\mathrm{Pt} / \mathrm{Pt}(111)$ changes significantly if there are very small amounts of $\mathrm{CO}$ in the residual gas pressure during deposition [200, 232]. At $400 \mathrm{~K}$ and $p_{\mathrm{CO}}=1 \times 10^{-9}$ mbar, the triangles are bound by $\mathrm{A}$ steps, that is, they are turned by $60^{\circ}$. At higher deposition temperature, the effect of CO is less prominent because it desorbs. Note that also the island densities are sensitive, even to much lower CO partial pressures [232].

The mechanism responsible for the triangular island shapes is as follows. In the kinetic regime, the cluster shape is determined by the growth rate perpendicular to $\mathbf{A}$ and $\mathbf{B}$ steps, that is, by the rate with which adatoms accumulate at both steps. The slowly growing facets prevail in the final crystal shape, whereas the faster ones disappear during growth, as generally the case in crystal growth. The necessary condition for compact islands is that atoms can diffuse along steps. For different growth rates of differently oriented steps, atoms must be able to diffuse from one step around a corner to the next step with a different orientation. The rate-limiting step for this so-called corner crossing implies an atom going from a laterally twofold to a onefold coordination. This process can have slightly different energy barriers starting from A versus B steps, thus creating a net diffusion current around the corner. In general, the barriers for corner crossing are quite close to those for edge diffusion $[219,224]$ such that this process is turned on as soon as edge diffusion is turned on. However, a small difference between the two suffices to change the entire island shape. In the case of $\mathrm{Pt}(111)$ homoepitaxy, the atoms leaving $\mathbf{B}$ steps and crossing over to A steps must be faster than the other way around. Thus atoms accumulate at $\mathbf{A}$ steps that grow out of the way and $\mathbf{B}$ steps remain $[88,233]$. The example of $\mathrm{Pt}(111)$ homoepitaxy shows that the island shape is a sensitive indicator for small energy differences; it is therefore a very accurate benchmark for $a b$ initio calculations.

At thermodynamic equilibrium, the adatom supply to the different steps is not decisive any more. Atoms can leave steps back into the 2D adatom gas on the terraces and condense again at a different location. They repeat this process until the total energy of the island is minimized. Under these conditions, the shape is given by the free energies $\gamma$ of $\mathbf{A}$ and $\mathbf{B}$ steps. These energies are reflected in the lengths of these facets according to the Wulff construction [234] (for 3D equilibrium shapes see Chapter 8, Volume 2). In monolayer-deep vacancy islands, the equilibrium shape can be reached more easily than in adatom islands, as the atoms evaporating from steps are confined to the vacancy and therefore they can be exchanged between the steps while keeping the vacancy area constant. Figure 20.18a shows an STM image of a vacancy island on $\mathrm{Al}(111)$ created by lowtemperature sputtering of about half a monolayer and subsequent annealing [29]. The $\mathbf{B}$ steps are significantly longer and also straighter than the A steps.

Figure 20.18b shows as full line the vacancy island shape averaged over 10 STM images taken consecutively of the same vacancy island. This line is straight when approaching the polar angles of the three B steps, while it is smoothly curved in the angular regions of the three A steps. The inverted Wulff construction 
a)

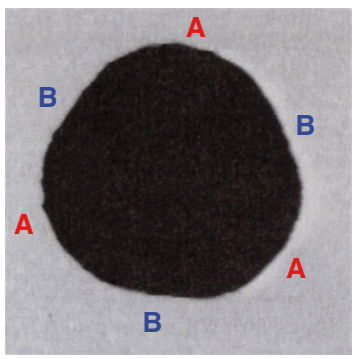

b)

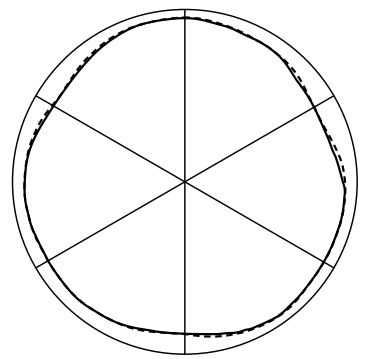

20.6 Coarsening of Monolayer Islands

c)

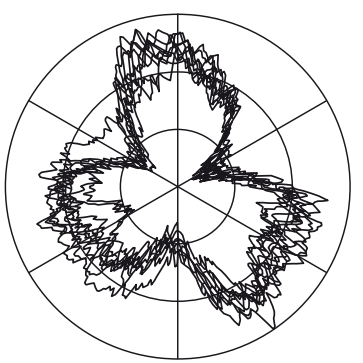

Figure 20.18 (a) STM image of monolayer-deep vacancy island on $\mathrm{Al}(111)$ (sputtering with $1 \mathrm{keV} \mathrm{Ne} e^{+}$ions at $200 \mathrm{~K}$, annealing for $300 \mathrm{~s}$ to $425 \mathrm{~K}, T_{\text {STM }}=300 \mathrm{~K}$ ). (b) The solid circle represents the mean island shape of island (a) obtained by averaging over 10 STM images of it; the dashed circle represents the polar plot of the step free energy $\delta(\Phi)$. (c) Step positions in subsequent STM images (of maximum island radius center $85 \%$, first ring $90 \%$, second ring $95 \%$, and outermost ring $100 \%$ ). (Adapted from Ref. [29].)

leads to the polar plot of the step free energy $\delta(\Phi)$ shown as dashed line. It gives $\delta_{\mathrm{B}} / \delta_{\mathrm{A}}=0.951 \pm 0.005$ [29] in good agreement with DFT predictions [102]. A similar analysis has been performed for $\mathrm{Pb}(111)$-terminated crystallites grown on $\mathrm{Cu}(111)$ [235, 236]. For further examples of $\mathrm{Ag}, \mathrm{Cu}, \mathrm{Pt}$, and $\operatorname{Ir}(111)$ see Ref. [29].

Further information is obtained when comparing the step positions between the 10 subsequent STM images. Figure 20.18c shows them on a strongly amplified scale, where the outer ring represents the largest observed island radius and the center $85 \%$ of it. All steps fluctuate at $300 \mathrm{~K}$. This a good sign, as it testifies that the steps are nowhere pinned by adsorbates and that we are indeed close to equilibrium because the shape is not given by kinetic limitations but by the result of steady-step atom exchange between opposite step orientations. It is clearly seen that A steps fluctuate more strongly. A fluctuation of the radial step position implies creation of kinks or of adatoms attached to an otherwise straight step. On an A step, a kink is a short section of a B step, and an adatom creates two such sections, while kinks and adatoms on $\mathbf{B}$ steps involve short sections of A steps. This immediately shows that step fluctuations of the microfacet with the lower step energy cost more energy, as they involve short sections of the costly step orientation [29]. Therefore, the observed stronger fluctuations of A steps are expected, as they involve short sections of the energetically favored step orientation. Note also that similar arguments give rise to a different temperature dependence of the two step free energies.

20.6

\section{Coarsening of Monolayer Islands}

We have seen that it is easier to reach thermodynamic equilibrium shapes for vacancy than for adatom islands because the former preserve their volume during evaporation of step atoms into the 2D adatom gas on the terrace. We also discussed 
that small clusters are mobile and that this mobility modifies the nucleation island densities. Both phenomena, the 2D evaporation from steps and the diffusion of entire clusters, give rise to island coarsening and will be discussed in this section. In addition to the fundamental interest in understanding the scaling exponents and the underlying atomic processes, coarsening opens ways to modify nanostructures on surfaces in a desired way.

\subsection{1}

\section{The Ostwald Ripening}

The Ostwald ripening [237] is the coarsening of an ensemble of different-sized 3D clusters embedded into a host (solid, liquid, gas). This process is driven by minimization of the overall cluster interface with the surrounding phase that has the surface or interface free energy $\gamma$. In an atomic picture, the clusters evaporate atoms from their surface and these atoms diffuse into the surrounding medium and condense either back to the same or to another cluster. As small clusters have a significantly higher fraction of surface atoms, statistical fluctuations of their size will have larger amplitude and the probability that they dissolve is higher than that for larger clusters. In a thermodynamic picture, the Ostwald ripening is driven by the local curvature $\kappa$ of the interface. The Gibbs-Thomson equation relates $\kappa$ to a change in the atoms' chemical potential $\Delta \mu$

$$
\Delta \mu=\gamma \kappa
$$

This implies mass flow from small clusters that have high local curvature to large ones leading to ripening. In addition, changes in local curvature of initially rough interfaces between two nonmiscible phases get smoother on annealing, or with time.

Through the availability of surface microscopy at the atomic level, this phenomenon has received considerable attention in two dimensions [198, 238]. The continuum theory of the Ostwald ripening $[239,240]$ has been applied to surfaces [241, 242], where it leads to the following expression for the temporal evolution of the area $A$ of an island with radius $r$

$$
A(t, T)=-\beta\left(\rho(r)-\rho_{\mathrm{eq}}(r)\right)
$$

where $\rho(r)$ is the adatom density next to the island perimeter. The equilibrium adatom density required to maintain this island at steady size is derived from the Gibbs-Thomson relation for circular islands with isotropic step free energy to $\rho_{\text {eq }}(r)=\rho_{\infty} \exp \left(\gamma / k_{\mathrm{B}} T n r\right)$. The difference between this and the real density at the perimeter is the driving force of ripening. The rate $\beta$ is composed of step evaporation, terrace diffusion, and step condensation barriers, as well as the corresponding preexponential factors. In many metal-on-metal systems, there is no barrier for incorporation of atoms into ascending steps. Equation 20.10 then reads

$$
A(t, T)=-\frac{2 \pi D \rho_{\infty}}{n \ln \left(\frac{R}{r}\right)}\left(\mathrm{e}^{\frac{\gamma}{k_{\mathrm{B}} T r r}}-1\right)
$$




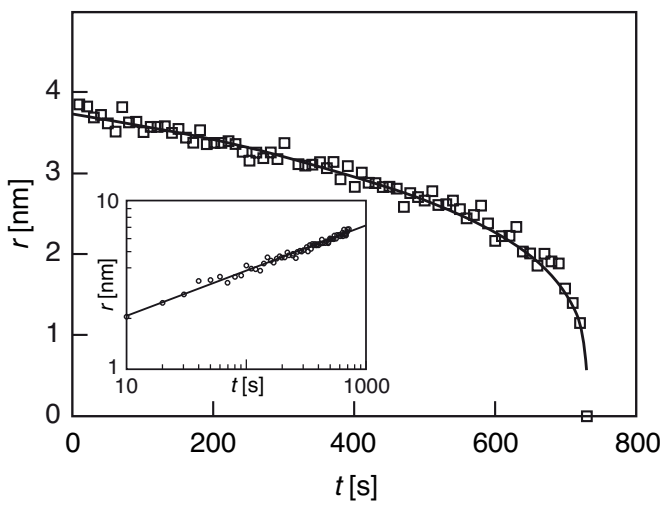

Figure 20.19 Temporal evolution of the radius of monolayer-high $\mathrm{Ag}$ islands on $\mathrm{Ag}(111)$ derived from sequential STM imaging ( $T=300 \mathrm{~K})$. (Adapted from Ref. [243].)

Note that here we label $D$ a diffusion constant that also includes the barrier for evaporation from the step onto the terrace, $n$ is the density of substrate unit cells, $\rho_{\infty}$ is the equilibrium adatom concentration on the terrace, and $R$ is the radius from the island center where $\rho$ takes on $\rho_{\infty}$. For $r \gg \gamma / k_{\mathrm{B}}$ Tn, it suffices to keep the leading terms in the exponential series. Neglecting the size dependence in the prefactor of Equation 20.11, one readily derives $r(t) \propto-t^{x}$ with $x=1 / 3$. STM inspection of the island decay at $300 \mathrm{~K}$ for $\mathrm{Ag} / \mathrm{Ag}(111)$ has enabled to follow $r(t)$, see Figure 20.19 for one island [243]. The double-logarithmic plot in the inset shows the scaling behavior. Averaging over 20 islands led to an exponent of $x=0.27 \pm 0.05$. This somewhat smaller value is attributed to the islands being small compared with the limit where a simple power law is justified. With an upper bound of $\gamma=0.2 \mathrm{eV}$ atom $^{-1}$ for the line tension, one obtains unity in the exponent of Equation 20.11 for $r=3 \mathrm{~nm}$. Therefore, within the error bar, the Ostwald ripening for $\mathrm{Ag} / \mathrm{Ag}(111)$ follows the exponent derived without step attachment barriers. One calls this case diffusion limited, where diffusion includes step detachment.

Attachment barriers can be introduced using a step incorporation coefficient $\kappa$ (1 for attachment without barrier and 0 for infinite barrier). This leads to the following expression for $r \gg \gamma / k_{\mathrm{B}} \operatorname{Tn}[244]$

$$
A(t, T)=-\frac{2 \pi D \rho_{\infty} \gamma}{n^{2} k_{\mathrm{B}} T} \frac{1}{\ln \left(\frac{R}{r}\right)+\frac{a}{\kappa r}}\left(\frac{1}{r_{\mathrm{c}}}-\frac{1}{r}\right)
$$

with $r_{\mathrm{c}}$ being the radius where the island size stays constant under the given parameters. For $\kappa=1, a / \kappa r$ is small compared with $\ln (R / r)$, and one finds Equation 20.11 in its large $r$ limit. The Ostwald ripening for $\operatorname{Si}(100)$ surfaces showed attachment barriers [238] related to the fact that attachment to a $\mathrm{Si}(100)$ step requires concerted motion of two neighboring Si dimers [245]. In the limit $r \ll r_{\text {c }}$, Equation 20.12 predicts a scaling exponent of $x=1 / 2$ for $r(t)$, respectively, a linear decrease in $A(t)$.

The area of small $\mathrm{Cu}$ islands on $\mathrm{Cu}(100)$ has indeed been observed to linearly decay with time, and this is independent of their environment [244]. Figure 20.20a 
a)

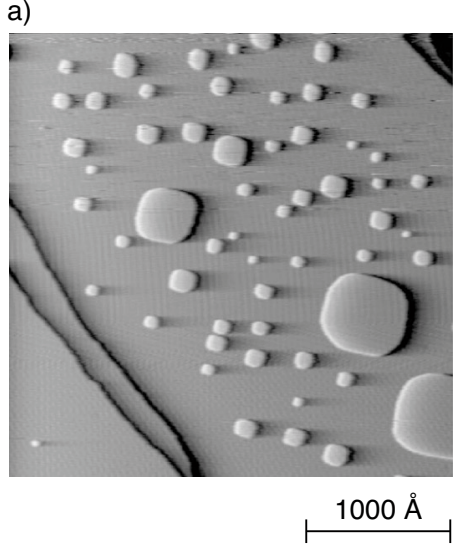

b)

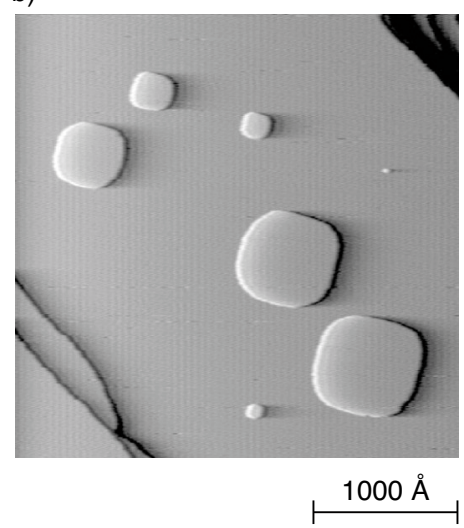

12:01:45

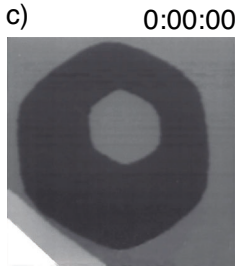

4:03:59
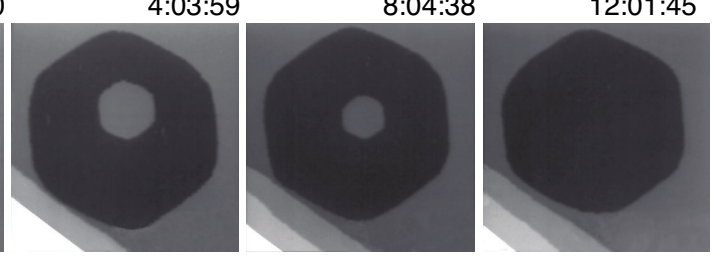

$100 \mathrm{~nm}$

d)

0:00:00 0:13:42

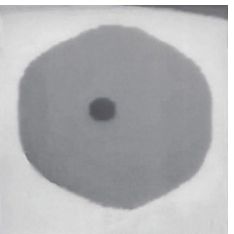

$0: 41: 06$

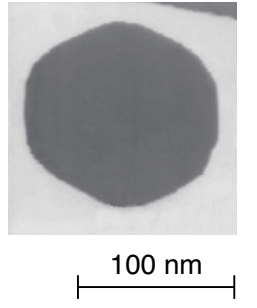

Figure $\mathbf{2 0 . 2 0}$ (a) and (b) STM images showing ripening of an assembly of about $60 \mathrm{Cu}$ adatom islands on $\mathrm{Cu}(100)$. A period of $5 \mathrm{~h} 33 \mathrm{~min}$ has passed between (a) and (b) images, with the substrate held at $343 \mathrm{~K}$ [244]. The time and temperature dependent decay of Ag adatom (c) and vacancy islands (d) placed into vacancy islands on $\mathrm{Ag}(111)$ permits determination of the activation barrier of atom detachment from steps and of the additional barrier for descending a step, (c) $T=300 \mathrm{~K}$ and (d) $T=360 \mathrm{~K}[112]$.

shows 60 different-sized adatom islands on a large terrace, a situation where ripening is essentially local, that is, the local coverage remains conserved and is redistributed between the islands. The size evolution of all islands, apart from one having undergone coalescence with another one, could perfectly be described by Equation 20.12, whereas $\kappa=0$ led to disagreement. The astonishing fact that a simple metal system exhibits significant attachment barriers was attributed to ripening being dominated by vacancy and not by adatom motion [244]. Vacancy attachment has an additional barrier compared with vacancy diffusion because it requires the 
concerted motion of two atoms. Vacancy motion has been confirmed for this system by tracing the displacement of substitutional In atoms on $\mathrm{Cu}(100)[246,247]$.

The experiments for $\mathrm{Ag} / \mathrm{Ag}(111)$ have been repeated for adatom and vacancy islands placed within a big vacancy island (Figure 20.20c,d) [112]. This eliminates less well defined parameters, such as the distance where $\rho$ has its asymptotic limit $\rho_{\infty}$ and thereby it gives access to more system parameters such as the step free energy, the energy barrier for step detachment, and the interlayer diffusion barrier.

The decay of an adatom island of radius $r$ within a vacancy island of radius $R$ is given by

$$
A(t, T)=-\beta \frac{\mathrm{e}^{\gamma / k_{\mathrm{B}} T n r}-\mathrm{e}^{-\gamma / k_{\mathrm{B}} T n R}}{\frac{a}{r}+\ln \left(\frac{R}{r}\right)+\frac{a}{R}}
$$

The authors first deduced $\gamma=0.75 \pm 0.15 \mathrm{eV} \mathrm{nm}^{-1}$, equivalent to $0.21 \pm 0.04 \mathrm{eV}$ atom $^{-1}$, from fitting decay rates of three adatom and four vacancy islands. The island area $A(t, T)$ showed the Arrhenius behavior from 240 to $310 \mathrm{~K}$ for $\beta=2 \pi D \rho_{\infty} / n$, highlighting the role of $D$ in this term. With $\beta \propto \nu_{0} \mathrm{e}^{-\mathrm{E}_{\mathrm{E}} / k_{\mathrm{B}} T}$, the authors found the energy barrier and attempt frequency for atom detachment from steps onto terraces $E_{\mathrm{d}}=0.71 \pm 0.03 \mathrm{eV}$ and $v_{0, \mathrm{~d}}=10^{12 \pm 0.6} \mathrm{~Hz}$. For the geometry shown in Figure 20.20d, the filling and hence the decay of the small vacancy island is hindered by the interlayer diffusion barrier. This barrier is determined as $\Delta E_{\mathrm{s}}=0.13 \pm 0.04 \mathrm{eV}$, consistent with former measurements reporting $\Delta E_{\mathrm{s}}=0.120 \pm 0.015 \mathrm{eV}[108]$.

Coarsening by the Ostwald ripening is a means of preparing compact islands with narrow size distributions. The average size can be adjusted from $\langle s\rangle=3$ $-10^{3}$ atoms by varying the annealing temperature [198]. This helps prepare large ensembles of nanostructures with well-defined size giving access to the evolution of chemical and physical properties with size [248, 249].

The starting point for the controlled increase in mean cluster size by ripening is a large density of small clusters, mostly dimers, which are prepared by deposition of $0.1 \mathrm{ML}$ at a temperature chosen such that there is little mobility in the time of deposition $\left(D / F<10^{3}\right)$. In this postnucleation regime, monomers diffuse toward each other after deposition leading to a mean cluster size of $\langle s\rangle \sim 3$ atoms $[88,126]$. The same result is obtained when depositing at temperatures where diffusion is frozen and subsequently gently annealing the surface to activate diffusion. After preparation of the small clusters, their densities are monitored by STM as a function of annealing temperature. This yields the average size because the coverage $\Theta$ is a known constant.

The Ag islands on Pt(111) shown in Figure 20.21a contain on average 14 atoms and are compact spherical. In Figure 20.21b, they contain 200 atoms and adopt a quasi-hexagonal shape. The largest ones clearly show the different lengths of the A and B steps. This shape becomes even more evident upon annealing to $280 \mathrm{~K}$ $(\langle s\rangle=800$ atoms, not shown) and can be considered as the equilibrium shape of a 2D cluster for that system because further annealing leads to island decay, which is believed to be promoted by compressive stress resulting from the lattice mismatch between Ag and Pt [251, 252]. 
a)

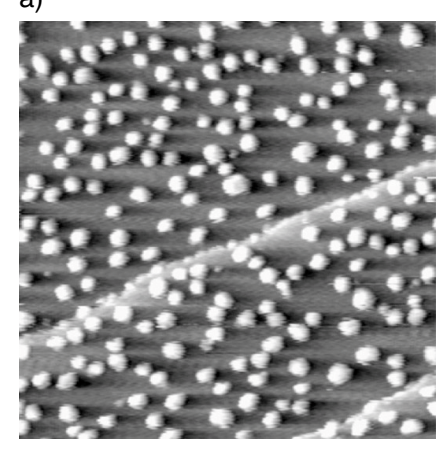

$100 \AA$

c)

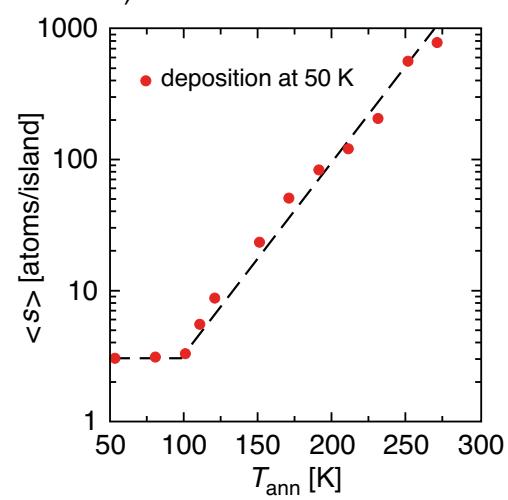

b)

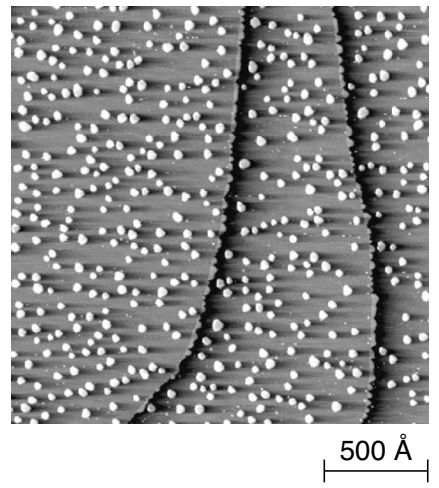

d)

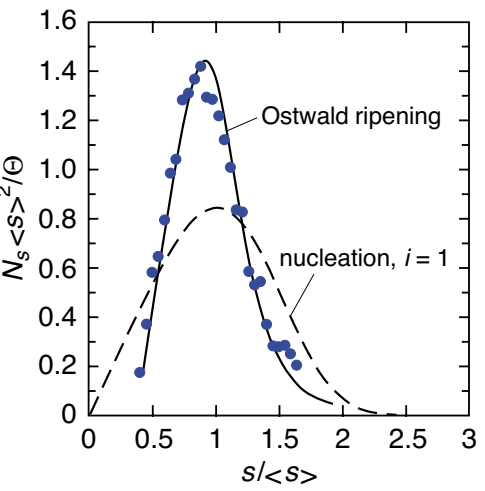

Figure 20.21 (a) and (b) STM images of Ostwald ripening for $\mathrm{Ag} / \mathrm{Pt}(111)$ creating compact $2 \mathrm{D}$ islands with their mean size $\langle\mathrm{s}\rangle$ controlled by the annealing temperature $(\Theta=0.10 \mathrm{ML}$, $T_{\text {dep }}=50 \mathrm{~K}$, (a) $T_{\text {ann }}=140 \mathrm{~K}$, (b) $T_{\text {ann }}=230 \mathrm{~K}$ ) [198]. (c) $\langle$ s $\rangle$ stays constant until $T_{\text {ann }}=100 \mathrm{~K}$, from where on it exhibits an exponential increase. (d) Island size distributions for Ostwald ripening (solid line, guide to the eye) [250] and nucletion (dashed line, theoretical $i=1$ scaling curve) [141].

Figure 20.21c shows the evolution of $\langle s\rangle$ with annealing temperature. It exhibits a plateau followed by an exponential increase. The constant regime implies that the most fragile objects in the population, namely, the dimers (and on square lattices also the trimers), neither dissociate nor diffuse, as both would lead to coarsening. The exponential increase is reminiscent of the Ostwald ripening. The temperature threshold of its onset thus defines the $i=1$ regime and yields the dimer bond energy $E_{\mathrm{b}}=150 \pm 20 \mathrm{meV}$ under the assumption $E_{\text {diss }}=E_{\mathrm{m}}+E_{\mathrm{b}}[88]$.

As an advantage to island nucleation, the size distributions obtained from the Ostwald ripening are significantly sharper. The STM images in Figure 20.21a,b already show this qualitatively. The size distribution in Figure 20.21d shows that the HWHM decreases from $\sigma=0.55$ for homogeneous nucleation at $i=1$ to $\sigma=0.30$ for coarsening. This is wider than $\sigma=0.20$ reported for nucleation on 
a periodic template [170]. However, the Ostwald ripening is also significantly simpler, as it works on a homogeneous substrate. Similar to nucleation, the island size distributions obtained from the Ostwald ripening at various temperatures [28] become congruent when scaled the same way as for nucleation.

\subsection{2}

\section{The Smoluchowski Ripening}

A second mechanism of island coarsening is the diffusion of entire islands and their coalescence. This mechanism can dominate the Ostwald ripening, especially for systems with high cohesive energy, or for very low surface adhesion and therefore also low diffusion barriers, such as metal islands on graphene or $h$-BN.

We illustrate an example where this ripening process has been observed starting from single transition metal adatoms on a $\mathrm{Pt}(111)$ surface [253]. Every cluster size has its characteristic onset temperature of diffusion, giving rise to a stepwise increase in the mean island size as a function of annealing temperature, as shown for the case of Co in Figure 20.22. The curves for Fe and Pt have their plateaus at exactly the same mean sizes; however, the respective onset temperatures are system specific. They give access to the cluster diffusion energies of each size, as we will illustrate later for $\mathrm{Co} / \mathrm{Pt}(111)$. The height of the respective plateaus can be understood as follows. All monomers start to diffuse simultaneously at their onset temperature and form dimers and trimers. The latter result when a monomer is close to two monomers that have already met. Once the dimers start to diffuse, they might meet one of their own or join an immobile trimer giving rise to a mixture of tetramers, pentamers, and remaining trimers. At the next higher temperature, these trimers start to move and join the other islands.

The black curve shows KMC simulations for monomer and dimer diffusion. In order to keep the number of parameters low, a common preexponential factor $v_{0}$ has been used, leaving the energy barriers of the two processes as the only two remaining fit parameters. Note that the step heights cannot be adjusted. Nevertheless, they are exactly reproduced, confirming the interpretation that this is the Smoluchowski ripening with the peculiarity of one atom increment sizedependent onset temperatures for cluster diffusion. The step from $\langle s\rangle=1.0$ to 2.5 atoms at $65 \mathrm{~K}$ gives $E_{\mathrm{m}}=210 \pm 20 \mathrm{meV}$. This value is in excellent agreement with $E_{\mathrm{m}}=200 \pm 10 \mathrm{meV}$ deduced from the Arrhenius of $n_{x}$ for that system $[254,255]$ and with $E_{\mathrm{m}}=200 \pm 30 \mathrm{meV}$ measured with low- $T$ AFM by integrating the force needed to displace the atoms over the bridge site times the distance [256]. The attempt frequency had to be set to $v_{0}=1.5 \times 10^{15} \mathrm{~Hz}$, which is quite high compared with the common value of $v_{0}=10^{13} \mathrm{~Hz}$, but it is close to the value of $v_{0}=1 \times 10^{14 \pm 0.5} \mathrm{~Hz}$ found from the density scaling [255].

The following step from $\langle s\rangle=2.5$ to 4.5 atoms at $95 \mathrm{~K}$ gives a dimer diffusion barrier of $E_{\mathrm{m}, 2}=270 \pm 30 \mathrm{meV}$. As trimer diffusion is linked to several different atomic processes in KMC simulation, thus implying multiple parameters, the last step associated to the diffusion of trimers has been simulated by integration of the rate equations from mean-field nucleation theory. The result of these simulations 
is shown as the gray curve. It reproduces the KMC result for the first two steps and the experimentally observed third step at $160 \mathrm{~K}$. The resulting trimer migration barrier is $E_{\mathrm{m}, 3}=440 \pm 30 \mathrm{meV}$ [253]. After this step, the mean size is 6.5 atoms in the simulation, coming from the coexistence of tetramers, pentamers, hexamers, and a very small amount of heptamers.

We underline that this sequence of atom-by-atom size-dependent cluster diffusion barriers will always give rise to the steps we have discussed here for the example of $\mathrm{Co} / \mathrm{Pt}(111)$ and that have also been observed for $\mathrm{Pt}$ and $\mathrm{Fe}$ on the same substrate [253]. Evidently, if two following cluster sizes start to move at once, one step will be suppressed. Note that also size-selected metal clusters deposited on graphene undergo the Smoluchowski ripening (F. Esch and U. Heiz, personal communication). Upon reaching a certain cluster size, the Ostwald ripening will compete with the Smoluchowski ripening. The critical size for this depends on the cohesive energy of the cluster element and on the corrugation of the interaction potential with the surface. The increase in the mean size observed for $\mathrm{Co} / \mathrm{Pt}$ for $T_{\text {ann }} \geq 220 \mathrm{~K}$ in Figure 20.22 might already be that regime.

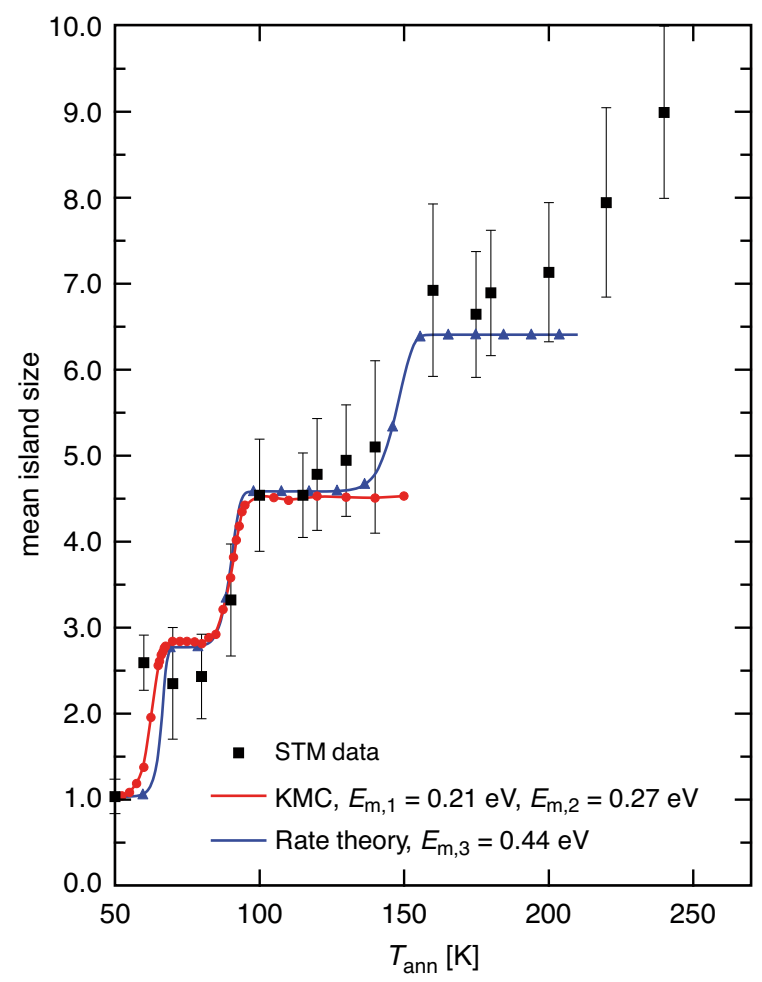

Figure 20.22 Mean island size in atoms as a function of annealing temperature derived from STM for Co/Pt $(111)\left(\Theta=0.01 \mathrm{ML}, t_{\mathrm{ann}}=1 \mathrm{~h}\right)$. Red curve shows KMC simulations with monomer and dimer diffusion; blue curve represents integration of mean-field rate equations considering in addition trimer diffusion $\left(v_{0}=1.5 \times 10^{15} \mathrm{~Hz}\right.$ for all processes) [253]. 
20.7

\section{Growth Morphologies}

At this point, we leave the submonolayer part of this chapter and turn our attention to the growth morphologies that form in the multilayer thickness regime and discuss their origin. Subsequently, we close by describing methods that enable growth manipulation such as to lead the desired flat film surfaces and atomically sharp interfaces.

\subsection{1}

\section{Growth Oscillations}

There are three surface morphologies that appear during the course of thin epitaxial film growth, and each of them is associated with its own thicknessdependent roughness evolution. Figure 20.23a shows that the sample stays all the

a)

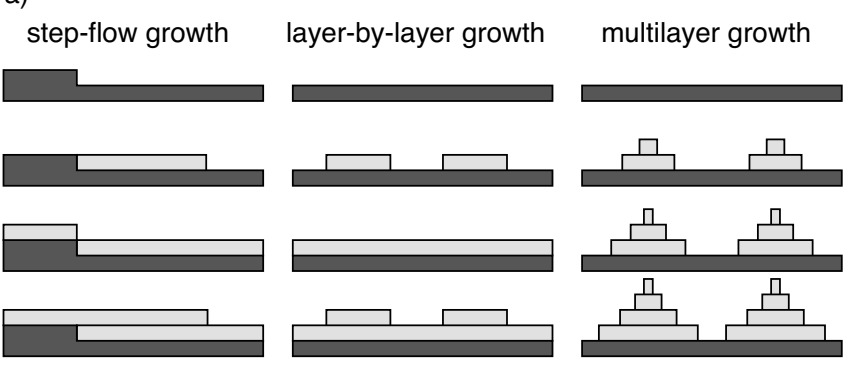

b)

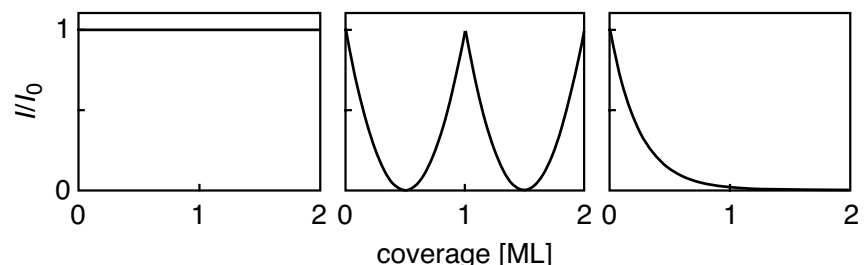

c)

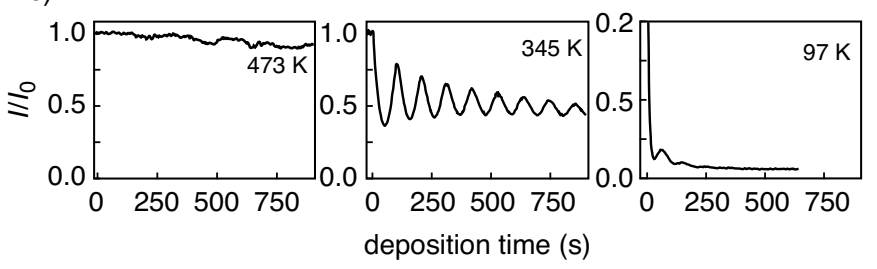

Figure 20.23 (a) Evolution of thin film growth morphology with coverage. (b) The normalized intensity of a diffraction signal recorded under antiphase conditions for one atomic layer height difference. (c) Antiphase-reflected intensity obtained from $\mathrm{He}$ atom scattering for $\mathrm{Cu}$ growth on a $\mathrm{Cu}(100)$ surface at various deposition temperatures [257]. Step-flow growth is observed at $473 \mathrm{~K}$, layer-by-layer growth at $345 \mathrm{~K}$, and kinetic roughening at $97 \mathrm{~K}$. 
time flat when the film grows by step flow that we had introduced above to define homo- and heterogeneous nucleation. Layer-by-layer growth implies a roughness maximum each time the thickness reaches half-integer monolayers, while at integer monolayers, the surface is as flat as the initial substrate. Multilayer growth leads to a monotonically increasing surface roughness with film thickness. The term multilayer growth has to be understood in the sense of many layers being open and thus growing simultaneously, while in layer-by-layer growth, there are only two open layers, and in step flow only one. The three film morphologies are caused by kinetics rather than thermodynamics and therefore should not be confused with the growth modes discussed earlier in this chapter.

The surface roughness can be probed in situ during growth by diffraction techniques using electrons, X-rays, or a coherent beam of helium atoms with thermal energy. The latter technique is referred to as He atom scattering (HAS), see Chapter 8 in Volume 2. In all three methods, the contrast is obtained by adjusting wavelength and the incident angle in such a way that the two beams specularly reflected from subsequent atomic planes interfere destructively. Figure 20.23b shows the expected normalized intensity of a diffraction signal recorded under these antiphase scattering conditions. It stays constant for step flow, shows oscillations with constant amplitude for layer by layer, and an exponential decay for multilayer growth.

The signal measured with HAS for $\mathrm{Cu}(100)$ homoepitaxy is shown in Figure $20.23 \mathrm{c}$ for the three growth temperatures yielding the three cases discussed. At $473 \mathrm{~K}$, the reflected He intensity stays constant. At this temperature, all atoms may reach the substrate steps and the film grows without nucleation at all. At which temperature step-flow growth is expected can be estimated for a given system from its saturation island density. The mean distance a monomer travels before encountering one of its own or an existing nucleus is the mean island spacing at saturation $\lambda \approx \sqrt{1 / \pi n_{x}} \approx(D / F)^{1 / 6}$, where the last equality applies to $i=1$. If $\lambda$ is larger than the terrace width, no islands are formed and all atoms reach the substrate steps to which they attach.

Very nice growth oscillations are obtained in our example of $\mathrm{Cu} / \mathrm{Cu}(100)$ at $345 \mathrm{~K}$. Comparing the respective Figure $20.23 \mathrm{~b}$ and c reveals that the oscillation amplitude is strongly damped in the experiment. This is due to growth imperfections on a length scale smaller than the coherence length of HAS. Perfect periodic monolayer oscillations of the surface roughness would appear when the film grows from nuclei that stay 2D during their growth until they coalesce. This implies that all atoms deposited atop the islands can descend until percolescence. Then the roughness has its maximum just before percolescence and a minimum when an integer layer is completed.

Multilayer growth is obtained for $\mathrm{Cu} / \mathrm{Cu}(100)$ at $97 \mathrm{~K}$. One sees a small peak at $1 \mathrm{ML}$, but the envelope of the reflected $\mathrm{He}$ atom intensity corresponds to the expected exponential decay. Multilayer growth is also referred to as kinetic roughening or mound formation. It involves the additional energy barrier $\Delta E_{\mathrm{s}}$ for an adatom to descend a step. Kinetic roughening occurs if, after the first layer nuclei have reached a critical radius $r_{c}$, atoms deposited atop can no longer descend 
quickly enough, leading to the critical monomer density atop the island for secondlayer nucleation. For dimers being stable nuclei, it suffices to have two monomers long enough present atop an island that they meet each other. The criterion for mound formation is $\lambda>2 r_{\mathrm{c}}$, that is, kinetic roughening occurs if islands grow to more than two times the critical radius before they coalesce, or in other words, if second-layer nucleation starts half-way before coalescence. For homoepitaxy with typical deposition fluxes and attempt frequencies, one can derive the criterion $\Delta E_{\mathrm{s}}+1 / 6 E_{\mathrm{m}}=E_{\mathrm{s}}-5 / 6 E_{\mathrm{m}}>4.8 k_{\mathrm{B}} T$, relating mound formation to the energy barriers of the two decisive atomic diffusion processes [29].

20.7.2

"Wedding Cakes"

In case of infinite step-edge barriers, no atom can descend and one obtains a Poisson distribution for the exposed coverages [258]. Pt(111) homoepitaxy at 440 $\mathrm{K}$ is almost a perfect realization of this scenario, also called statistical multilayer growth [259]. Figure 20.24 shows that the mound density is given by the initial island density, the number of open layers augments with $\Theta$, and top layer steps approach

a)

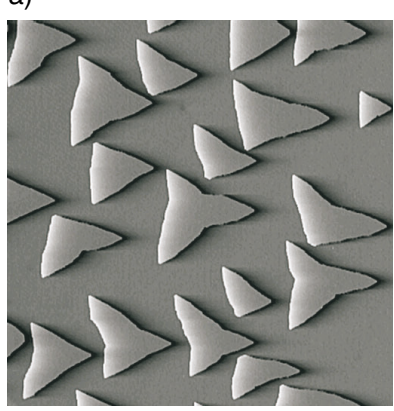

c)

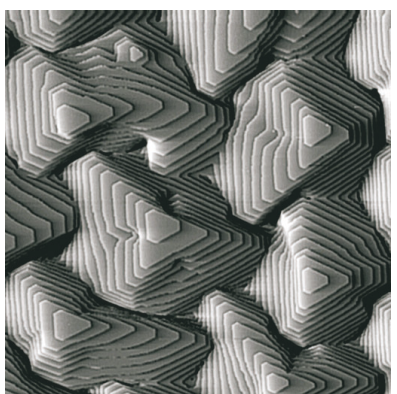

b)

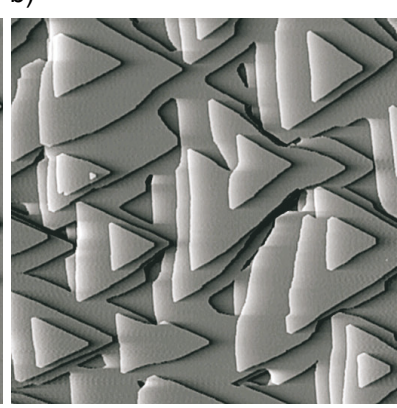

d)

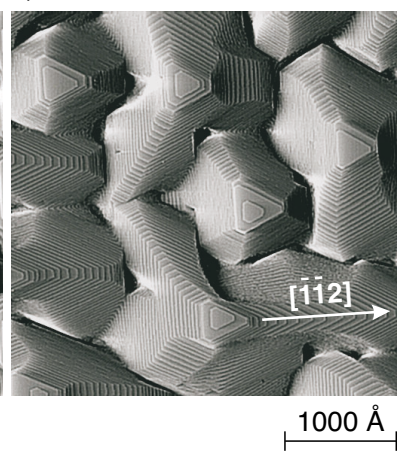

Figure 20.24 Growth morphology as a function of coverage for $\mathrm{Pt}(111)$ homoepitaxy at $T_{\text {dep }}=440 \mathrm{~K}: \Theta=$ (a) 0.35 , (b) 3, (c) 12 , and (d) $90 \mathrm{ML}\left(F=7 \times 10^{-3} \mathrm{ML} \mathrm{s}^{-1}\right)$. (Adapted from Ref. [259].) 
the perfect close-packed $\langle 1 \overline{1} 0\rangle$ orientation, while the ones of the first few layers are rougher and less well aligned to the close-packed directions [29]. This is quantified by the kink density going from 0.2 at $0.35 \mathrm{ML}$ down to 0.009 at $306 \mathrm{ML}$ [29]. For earlier examples of statistical multilayer growth see Refs [260, 261].

The layer distribution obtained from STM images such as Figure 20.24d corresponds quantitatively to the expected Poisson distribution, apart from the last view top layers that are significantly larger. This is due to finite interlayer diffusion being more important for the uppermost terraces, as on them, there are no ascending steps where the deposited atom might attach, and therefore, it explores the descending step many times until it eventually descends, even in the presence of large $\Delta E_{\mathrm{s}}$ values. One can introduce finite interlayer diffusion by allowing the growth of layer $n$ only when the preceding layer $n-1$ has reached a critical coverage $\Theta_{c}$ [29]. For the example of $\mathrm{Pt}(111)$ homoepitaxy at $440 \mathrm{~K}$, there is very good agreement with the experimental layer distributions for $\Theta_{c}=0.122 \mathrm{ML}$ translating into $\Delta E_{\mathrm{s}}=0.14 \mathrm{meV}[29]$.

The additional interlayer diffusion barrier strongly depends on the atomic step site where the descent takes place. On kinks, it can be close to 0 and on straight steps it is around $0.33 \mathrm{meV}$ in the case of $\mathrm{Pt}(111)$. Therefore, the layer- and deposition-temperature-dependent kink density leads to a thicknessand temperature-dependent effective interlayer diffusion barrier $\Delta E_{\mathrm{s}, \mathrm{eff}}\left(\Theta, T_{\mathrm{dep}}\right)$. With the aforementioned kink concentrations, one obtains from adding up the interlayer diffusion rates according to the abundance of kink versus straight step sites $\Delta E_{\mathrm{s}, \text { eff }}(0.35 \mathrm{ML}, 440 \mathrm{~K})=0.06 \mathrm{eV}$, whereas $\Delta E_{\mathrm{s}, \mathrm{eff}}(306 \mathrm{ML}, 440 \mathrm{~K})=0.18 \mathrm{eV}$. The interlayer barrier appearing in the criterion for mound formation is also an effective one.

The very small kink density of the highest layer islands is attributed to the fact that they are smaller than the first islands that have no bound for their growth other than the mean distance given by $n_{x}$. Therefore, the uppermost layer islands do not reach the critical length from which on adatom diffusion along the step is rate limiting and spatial fluctuations of lateral adatom arrival lead to shape instabilities with rough steps. A second reason is that the upper islands not only grow by lateral attachment of atoms but also much more than the lower layers by atom descend from top. As this descend takes place mostly at kinks, and as the descending atom just shifts the kink by one site but does not create a new one, the number of kinks is kept low.

\section{8}

\section{Growth Manipulation}

We discuss concepts of growth manipulation toward perfectly flat layers forming atomically sharp interfaces, as required in the majority of applications. We start by introducing the concept of layer-dependent adatom mobilities. In the following discussion of surfactants, we will see that their action very often is related to altering adatom mobilities on subsequent layers. We close by showing how layer-dependent 
mobilities and 2D growth can be enforced by periodic short energetic ion pulses accompanying growth.

20.8.1

\section{Layer-Dependent Adatom Mobilities}

The necessary condition for layer-by-layer growth in the kinetic regime is that every atom deposited on top of an island can reach the descending step. The sufficient condition is that it does descend before having had the chance to create a stable cluster on top with one or more of its own. Both conditions have to be met at any stage of growth. This brings us to the concept of layer-dependent adatom mobilities that is at the origin of growth manipulation [262].

We label the growing layer $n$. The mean island diameter of that layer at coalescence equals the average island separation given by the mean-free diffusion path $\lambda_{n-1}$ of adatoms of layer $n$ that diffuse on layer $n-1$ with rate $D_{n-1}$ (Figure 20.25). If the mobility on top of layer $n$ is larger or equal than that below, $\lambda_{n} \geq \lambda_{n-1}$, the atoms deposited on top reach the island edge at any stage of growth.

For homoepitaxy, $D$ is layer independent and the atoms can reach the island edge, but only a limited number of times. If there is a small extra barrier for edge descend the system grows 3D. Note that these are kinetic considerations. For a homoepitaxial system, it suffices to anneal the film and it will get flat. This underlines the difference of the kinetic roughening we are discussing here with the SK or the VM growth modes that are obtained at equilibrium.

For heteroepitaxy, there are generally layer-dependent mobilities resulting from layer-dependent surface structure and/or strain, which both have a strong effect

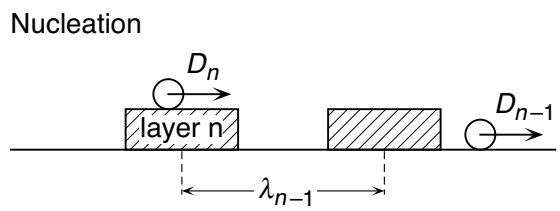

Coalescence, $D_{n}>D_{n-1}$ :

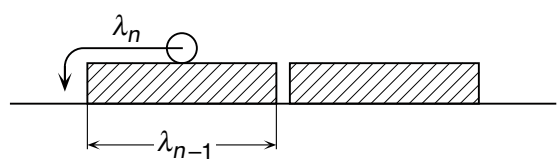

Coalescence, $D_{n}<D_{n-1}$ :

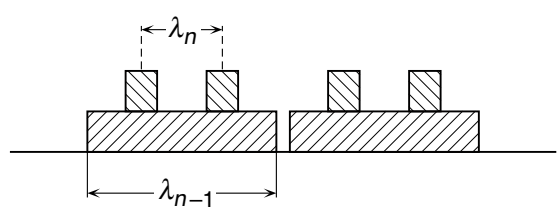

Figure 20.25 The concept of layer-dependent adatom mobilities [262, 263]. 
on $E_{\mathrm{m}}$ and $\Delta E_{\mathrm{s}}$. Strain can therefore promote layer-by-layer growth [71]. The most spectacular example are close to undamped growth oscillations induced in $\mathrm{Pt}(111)$ homoepitaxy at $T_{\text {dep }}=620 \mathrm{~K}$ [264]. They are caused by the Pt-adatom-induced reconstruction of the terraces [265]. The islands are unreconstructed and therefore adatoms diffuse very fast, while the diffusion on the terrace is slowed down by the reconstruction [266]. Therefore, one has $\lambda_{n} \gg \lambda_{n-1}$ giving the atoms atop the islands many attempts to descend the steps at the island edges leading to $2 \mathrm{D}$ growth even in the presence of a significant barrier for interlayer diffusion. The mobility on top of the growing layer can equally well be reduced by strain effects, see bottom of Figure 20.25, which inhibits layer-by-layer growth, even in the absence of an extra barrier for interlayer mass transport.

The concept of layer-dependent mobilities opens new ways to promote layer-bylayer growth by enhancing the mobility on top of the growing layer or by reducing it in the initial nucleation phase of each layer [262, 267, 268]. The action of surfactants has additional components to it; however, the concept of layer-dependent mobility is very useful for their description. We therefore first discuss surfactants and then turn to periodic perturbations of the growth conditions leading to layer-dependent mobilities inducing 2D growth.

20.8 .2

\section{Surfactants}

A surfactant is a foreign chemical species that supports layer-by-layer growth. A requirement of a surfactant is that it floats up onto the surface of the growing layer. Otherwise, it would get incorporated into and finally buried by the growing film. Thereby, its action would cease and the film properties deteriorate by the induced compositional change.

In the thermodynamic picture of epitaxial growth, the role of a surfactant is to lower the surface free energy of the film and thereby promote wetting and 2D growth [269]. However, often the action of a surfactant is required at temperatures where kinetics dominates the morphology. We have discussed earlier the criterion for mound formation, or kinetic roughening. As it contains the difference between $E_{\mathrm{s}}$ and $E_{\mathrm{m}}$, a surfactant can act on both these quantities, it might increase the barrier for surface diffusion or decrease the barrier for step descent. We have also pointed out that the latter is an effective barrier $E_{\mathrm{s} \text {,eff }}$. Therefore, there are two ways a surfactant can reduce it. The first is to reduce the energy barrier for the rate-limiting step-descent process; for example, a roll-down process can become an exchange process in the presence of the surfactant leading to a lower barrier. The second is to change the kink density at steps thereby inducing more step sites with lower $E_{\mathrm{s}}$, reducing $E_{\mathrm{s}, \mathrm{eff}}$. This can be identified by looking at the island shapes during growth in the presence and absence of the surfactant.

Altogether, this gives three ways by which a surfactant can act on the growth kinetics: increase in $E_{\mathrm{m}}$, reduce $E_{\mathrm{s}}$ for the relevant interlayer diffusion process, or reduce $E_{\mathrm{s} \text {, ff }}$ by reducing the adatom mobility along steps creating ramified islands with more kink sites. A surfactant may act in more than one of these three ways in 
parallel. Note that the decrease in $E_{\mathrm{m}}$ changes layer the moment the surfactant floats up. If this coincides with island coalescence or even layer completion, a surfactant induces the right layer-dependent mobilities favoring layer-by-layer growth.

Which element is the best surfactant for a given epitaxial system is still very often determined by trial and error. It is important that the surfactant is easy to remove; therefore, adsorbed gases such as $\mathrm{O}$ and $\mathrm{CO}$ are often used, but $\mathrm{Sb}, \mathrm{Pb}$, and In have also been used with success. For surfactants in semiconductor epitaxy, we refer to Ref. [270] and discuss here three examples from metal epitaxy. These examples are from homoepitaxy, and therefore, textbook examples illustrate the principle.

Figure 20.26 shows the stunning effect of $\mathrm{Sb}$ used as surfactant in the roomtemperature homoepitaxial growth of $\operatorname{Ag}(111)$ [271, 272]. The additional stepedge barrier of $\operatorname{Ag} / \operatorname{Ag}(111)$ is sufficiently high $[108,112,273]$ to induce mound formation at $300 \mathrm{~K}$. An STM image after deposition of $25 \mathrm{ML}$ is reproduced in Figure 20.26a and shows the 3D growth morphology resulting from kinetic

a)

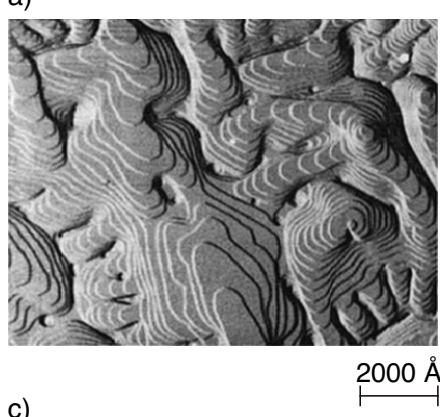

b)

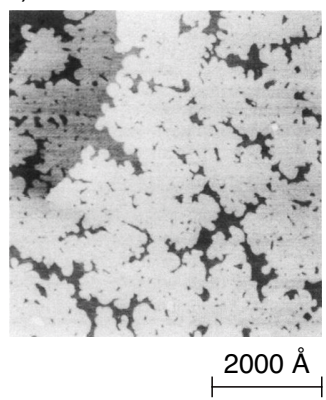

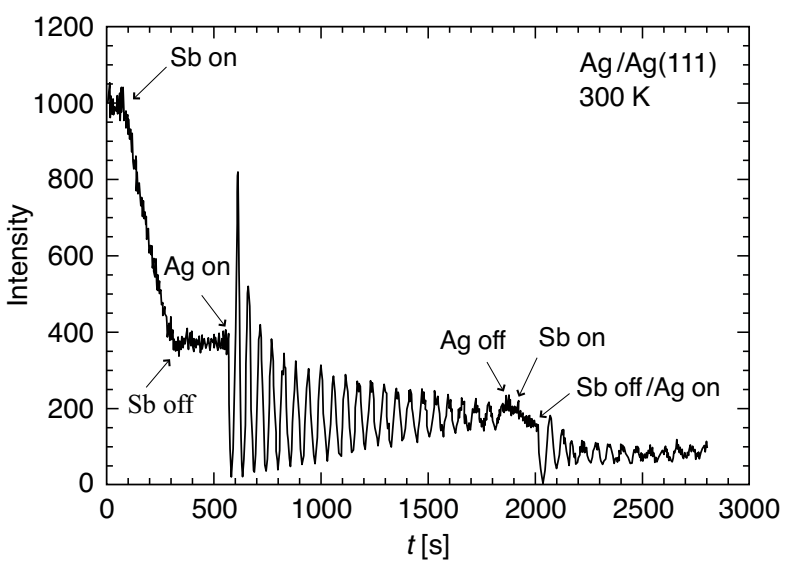

Figure 20.26 Layer-by-layer growth induced in $\mathrm{Ag}(111)$ homoepitaxy by $\mathrm{Sb}$ as surfactant $\left(T_{\text {dep }}=300 \mathrm{~K}\right)$. (a) $3 \mathrm{D}$ morphology after deposition of $25 \mathrm{ML} \mathrm{Ag}$ without surfactant. (b) $2 \mathrm{D}$ morphology with only two open layers $\left(\Theta_{\mathrm{Sb}}=0.08 \mathrm{ML} \Theta_{\mathrm{Ag}}=1.7 \mathrm{ML}\right)$. (Adapted from Ref. [271].) (c) X-ray reflectivity dosing Sb assurfactant at different stages of growth $\left(\Theta_{\mathrm{Sb}}=0.20\right.$ $M L$ in first adsorption step). (Adapted from Ref. [272].) 
roughening. Figure $20.26 \mathrm{~b}$ reveals that by adding a submonolayer coverage of $\mathrm{Sb}$, the film shows a perfect morphology for layer-by-layer growth. The surfactant effect of $\mathrm{Sb}$ is further evidenced in the surface X-ray reflectivity shown in Figure 20.26c. There are layer-by-layer oscillations each time $\mathrm{Sb}$ is dosed before growth [272]. The initial amplitude of the oscillations is large, however, they are damped after $15 \mathrm{ML}$ indicating that some of the Sb is buried during growth. STM revealed that $n_{x}$ increases by a factor of 40 if $\mathrm{Sb}$ is preadsorbed at $300 \mathrm{~K}$, where it forms $(\sqrt{3} \times \sqrt{3})$ adatom islands. This increase is still significant with $n_{x, \mathrm{Sb}}=6 n_{x, \text { clean }}$ when the precovered surface is annealed to $470 \mathrm{~K}$, where the $\mathrm{Sb}$ atoms isolate and take on substitutional sites [271]. Therefore, the main effect of $\mathrm{Sb}$ is the increase in $E_{\mathrm{m} \text {,eff }}$. DFT calculations confirm this picture as they show that $\mathrm{Sb}$ prefers substitutional sites on $\mathrm{Ag}(111)$ and that $\mathrm{Ag}$ adatom diffusion is slowed down by the repulsion exerted by the substitutional $\mathrm{Sb}$ atoms [274].

It is seen from comparison of Figure 20.26a and b, as well as from the submonolayer island shown in Figure 20.27b, that the islands have meandering steps in the presence of $\mathrm{Sb}$. Therefore, they have more kinks and thereby the surfactant also reduces $E_{\mathrm{s} \text {,eff }}$ in addition to its $E_{\mathrm{m} \text {,eff }}$ increase. The lower edge mobility behind this island shape transition might be caused by the chemical step heterogeneity due to the substitutional $\mathrm{Sb}$ atoms.

The island shapes for $\mathrm{Al}(111)$ homoepitaxy in the presence of preadsorbed oxygen islands are also much more ramified than without [133]. Figure 20.27a shows large Al islands with wide meandering branches with a shape similar to Ag islands formed on Pt(111) at $220 \mathrm{~K}$ discussed earlier. The oxygen is present in the form of $\mathrm{O}_{\mathrm{ad}}-(1 \times 1)$ islands $[275,276]$ that are imaged dark in Figure 20.27a. These islands act as repulsive point defects for the diffusing $\mathrm{Al}$ adatoms by which they change the effective diffusion barriers and island densities [133]. They also repel

a)

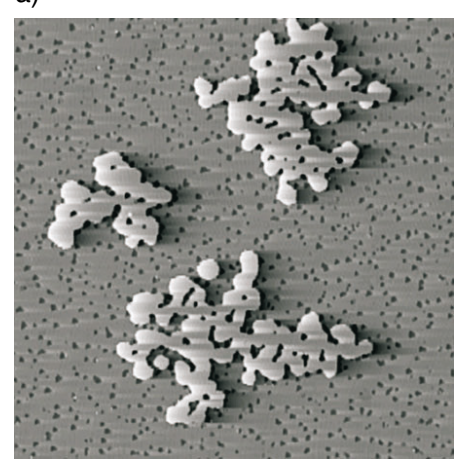

$2000 \AA$ b)

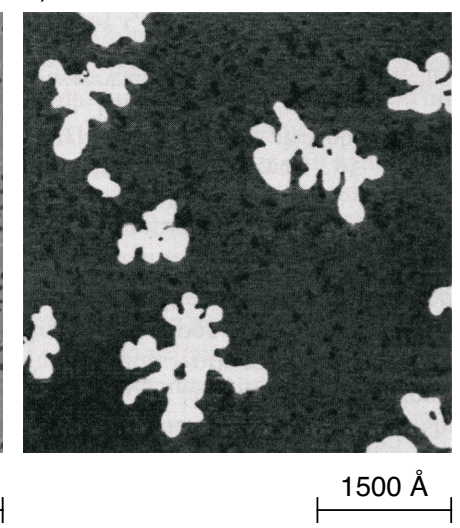

Figure 20.27 Adsorbate-induced modification of island shapes for (a) oxygen on $\mathrm{Al}(111)$ and (b) $\mathrm{Sb}$ on $\mathrm{Ag}(111)$. (a) $T_{\text {dep }}=300 \mathrm{~K}, \Theta=0.09 \mathrm{ML}$, exposure to 0.027 Langmuir $\mathrm{O}_{2}$ at $420 \mathrm{~K}$ leading to $0.1 \mathrm{MLO}_{\mathrm{ad}}$ islands. Kindly provided by T. Michely [29]. (b) $T_{\text {dep }}=300 \mathrm{~K}, \Theta_{\mathrm{Sb}}=0.08 \mathrm{ML}$, $\Theta_{\mathrm{Ag}}=1.1 \mathrm{ML}$. (Adapted from Ref. [271].) 
steps that grow around them leading to the observed meandering and possibly also to the voids in the islands.

Now we contrast an antisurfactant (CO) with a surfactant (O) for $\operatorname{Pt}(111)$ homoepitaxy. Very small amounts of $\mathrm{CO}$ adsorbed during growth raise $E_{\mathrm{s}}$ without significantly affecting $E_{\mathrm{m}}$ and the island compactness, therefore favoring mound formation. At saturation coverage of $0.5 \mathrm{ML}$, CO forms a $\mathrm{c}(4 \times 2)$ overlayer on $\mathrm{Pt}(111)$. It is seen from comparison of Figure 20.28a and $\mathrm{b}$ that this layer strongly increases $n_{x}$ at $T_{\text {dep }}=300 \mathrm{~K}$, namely, by a factor 5.5 [29]. Nevertheless, the growth morphologies after 1 and 5 ML (Figure 20.28e,h) are considerably rougher

a)

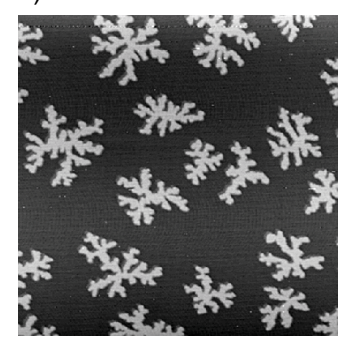

d)

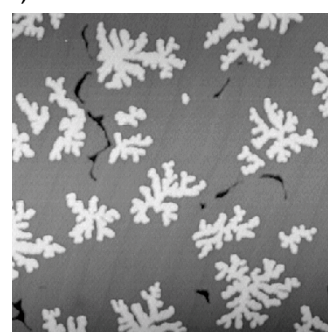

g)

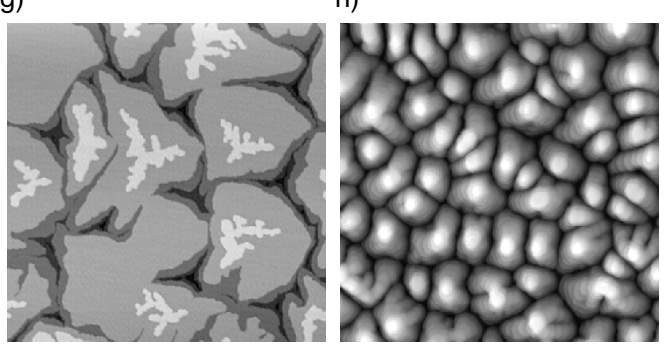

b)

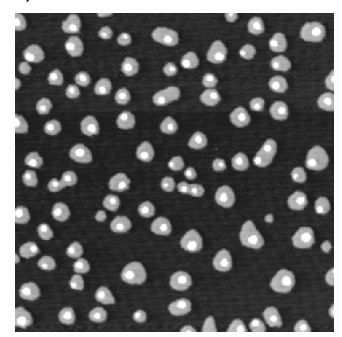

e)

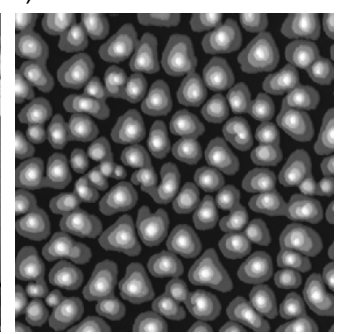

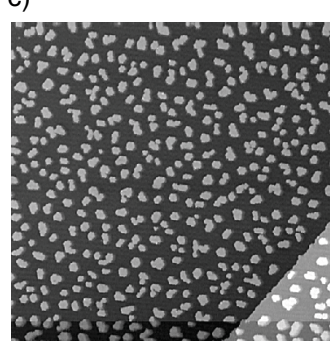

f)

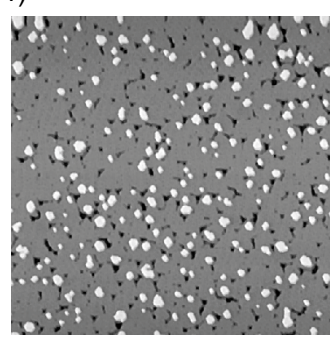

i)

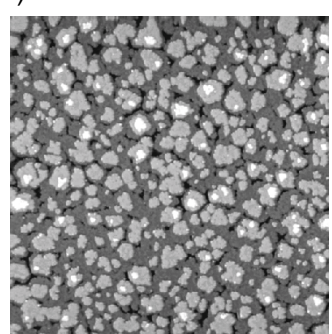

$300 \AA$

Figure 20.28 Submonolayer ( $\Theta=0.2 \mathrm{ML}$, first row), monolayer $(\Theta=1.0 \mathrm{ML}$, second row), and multilayer $(\Theta=5.0 \mathrm{ML}$, third row) morphologies for $\mathrm{Pt}(111)$ homoepitaxy at $300 \mathrm{~K}$, once clean (left column), once with $\Theta=0.50 \mathrm{MLCO}$ (middle column), and once with $\Theta=0.25 \mathrm{MLO}_{\mathrm{ad}}$ $\left(F=5 \times 10^{-3} \mathrm{ML} \mathrm{s}^{-1}\right)$. (Kindly provided by T. Michely and adapted from Ref. [29].) 
than those of the clean system. Therefore, the $E_{\mathrm{m}}$ increase must be more than counterbalanced by an $E_{\mathrm{S}}$ increase, and therefore, $\mathrm{CO}$ is an antisurfactant for Pt(111) homoepitaxy from very small up to saturation coverage on the growing film.

A partial pressure of oxygen in the background gas during $\mathrm{Pt} / \mathrm{Pt}(111)$ growth induces weak growth oscillations in HAS at temperatures where the clean system shows a monotonic decay of the specular He intensity [277]. This is indicative of a surfactant effect. Note that oxygen has also be identified as surfactant for $\mathrm{Cu} / \mathrm{Ru}(0001)$. For that system, the work function change has been used to monitor the growth oscillations $[278,279]$. On $\mathrm{Pt}(111)$, oxygen forms at saturation an adsorbed $\mathrm{p}(2 \times 2)$ layer with $0.25 \mathrm{ML}$ coverage. Its effect on the island density becomes apparent from inspection of Figure 20.28c, showing very many islands with 25 times higher density than without oxygen preadsorption. Under the assumption of identical critical cluster size and preexponential factors than the clean system, this $n_{x}$ increase converts into an $E_{\mathrm{m}}$ increase from 0.26 to $0.51 \mathrm{eV}$. Comparison of Figure 20.28d and f, respectively, with Figure 20.28g and i shows that the growth morphology is slightly more flat in the presence of oxygen than that in the clean case. Looking at the criterion for mound formation, one infers that the $\mathrm{O}_{\mathrm{ad}}$-induced increase of $E_{\mathrm{s}}$, if there is any, has to be less than $0.21 \mathrm{eV}$ in order to explain the observed growth improvement.

The surfactant effect of oxygen becomes more appreciable when growing at $400 \mathrm{~K}$. The morphologies after deposition of 5.0 ML with and without oxygen preadsorption are compared in Figure 20.29. Oxygen induces a close to perfect layer-by-layer morphology while 3D mounds with triangular shape are formed without surfactant. The inset in Figure 20.29b shows a surface grown under the same conditions as Figure 20.28i. The $\mathrm{p}(2 \times 2)-\mathrm{O}_{\mathrm{ad}}$ layer is clearly discerned

a)

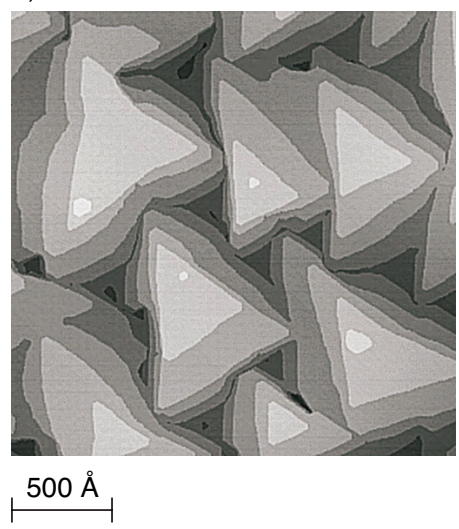

b)

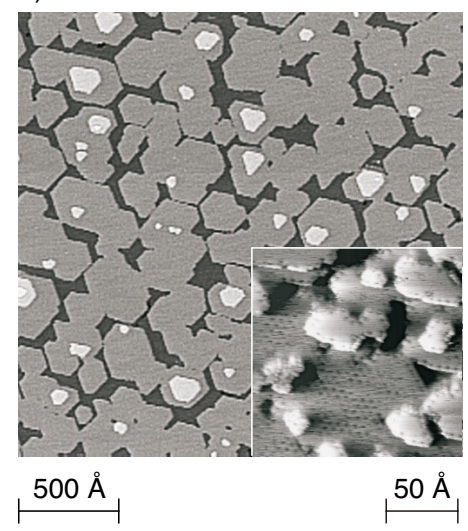

Figure 20.29 Surfactant effect of oxygen for $\mathrm{Pt}(111)$ homoepitaxy at $400 \mathrm{~K}(\Theta=5.0 \mathrm{ML})$. (a) 3D growth of clean system. (b) Flat growth after preadsorption of a saturation coverage of oxygen $(\Theta=0.25 \mathrm{ML})$. Inset: $\mathrm{O} / \mathrm{Pt}(111)-\mathrm{p}(2 \times 2)$ layer floats up after growth of $5.0 \mathrm{ML}$ at $300 \mathrm{~K}$. (Kindly provided by T. Michely and adapted from Ref. [29].) 
proving that it floats up, as requested for a surfactant. After growth, the O can entirely be removed by dosing $\mathrm{H}_{2}$ [29].

The effect of $\mathrm{O}$ has been studied in the unintentional presence of very small amounts of CO, thus mixing surfactant and antisurfactant [280]. In that case, the preadsorbed $\mathrm{O}_{\mathrm{ad}}$ layer prevents $\mathrm{CO}$ from adsorbing at steps and therefore leads to a reduction in $E_{\mathrm{s}}$ compared with the CO-contaminated case.

These examples illustrate the complexity of surfactant action. The simple decrease in $E_{\mathrm{S}}$, often wrongly concluded after the first observation of a surfactant effect, is the exception. The increase in $E_{\mathrm{m}}$, and the change of the kink abundance at steps, are much more often the way a surfactant operates in the kinetic growth regime. The first effect is related to layer-dependent adatom mobilities because the surfactant increases $E_{\mathrm{m}}$ on the layer where it is present, while atoms falling onto the islands growing on that layer have a higher mobility. Because we discussed here examples from metal homoepitaxy, we would like to point out the work using $\mathrm{Pb}$ for $\mathrm{Cu}(111)$ homoepitaxy $[281,282]$. For surfactants in metal heteroepitaxy, we refer to Refs $[278,283]$, and for their use in semiconductors, we refer to the review [270].

20.8 .3

Periodic Ion Bombardment

As we have seen, layer-by-layer growth is promoted if adatoms are more mobile on the topmost layer than on the one below. This augments the number of times they can reach the descending steps and therefore the probability that they eventually descend instead of creating a new nucleus with other atoms. Variation of the mobilities on subsequent layers of the growing epitaxial film can be provoked by the variation of external parameters with monolayer period. The mobility has to be decreased in the initial nucleation phase of each layer. This corresponds to an increase in $n_{x}$ and can be achieved by lowering $T_{\text {dep }}$ or by increasing $F$ during the initial nucleation phase [268]. The former is difficult because the substrate temperature cannot be varied very rapidly without significant heating power that might cause an unacceptable rise in background pressure. The flux can be varied more easily because one can install a second evaporator in order to alternate the regular with the high flux. However, the flux enters $n_{x}$ with an exponent of only $1 / 3$ for $i=1$, thus requiring typically two orders of magnitude higher fluxes to achieve the desired layer-by-layer growth.

A much more efficient and easier-to-implement method is a brief ion bombardment during the initial nucleation phase of each layer. It is seen from curve b in Figure 20.30 that this leads to pronounced layer-by-layer oscillations for $\mathrm{Ag} / \mathrm{Ag}(111)[262,284]$. This figure also compares the antiphase He reflectivity for continuous sputtering (curve c) with the one of the pristine system (curve a). As expected, continuous sputtering increases the film roughness beyond the one of clean $\mathrm{Ag} / \mathrm{Ag}(111)$. A nice demonstration that it is indeed the initial island density that gives rise to the observed effect on the film morphology has been provided by growing islands at $100 \mathrm{~K}(\Theta=0.05 \mathrm{ML})$ before continuing growth at $260 \mathrm{~K}$. The 


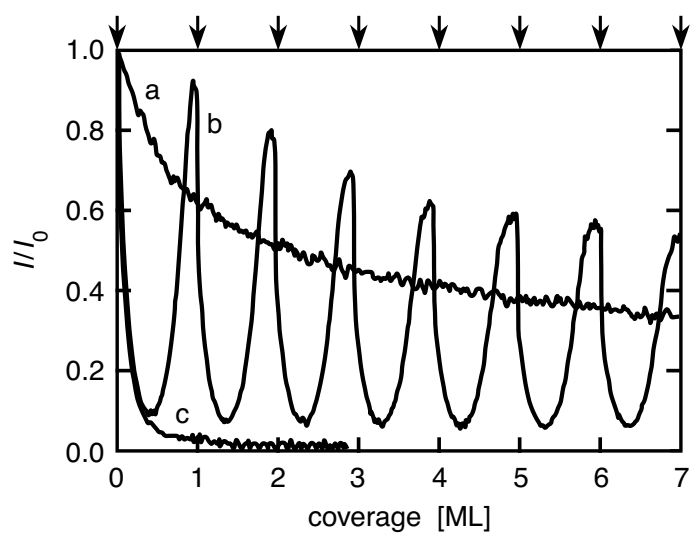

Figure 20.30 Curve (b), layer-by-layer growth induced in $\mathrm{Ag}(111)$ homoepitaxy by sputter pulses applied each time a monolayer is completed and a new layer nucleates, see arrows $\left(T_{\text {dep }}=300 \mathrm{~K}\right.$, $F=4.7 \times 10^{-3} \mathrm{ML} \mathrm{s}^{-1}, 600 \mathrm{eV} \mathrm{Ar}^{+}$sputter pulses, each removing $\left.0.014 \mathrm{ML}\right)$. (I/I 0 )-normalized antiphase $\mathrm{He}$ diffraction signal. Curve (a), kinetic roughening without growth manipulation. Curve (c), stronger roughening for continuous sputtering. (From Ref. [262].)

resulting He reflectivity is very similar to the one where $n_{x}$ has been increased by a brief sputter pulse and growth performed at $260 \mathrm{~K}$ [284].

The mechanism linking short energetic ion pulses to large island densities is the fact that they not only remove atoms from the surface (sputtering) but also create a large density of adatom islands [285]. Each ion removes roughly one atom and creates a large local density of adatoms around the ion impact site that condense into islands. The inset in Figure 20.31b shows the very high density of adatom islands

a)

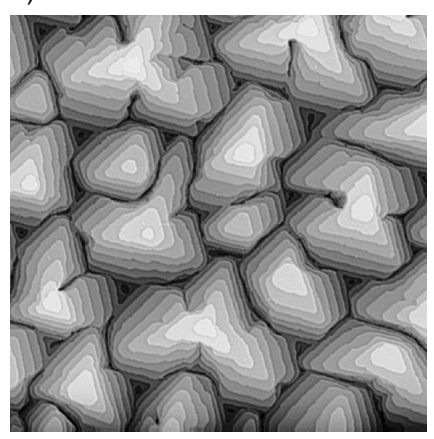

b)

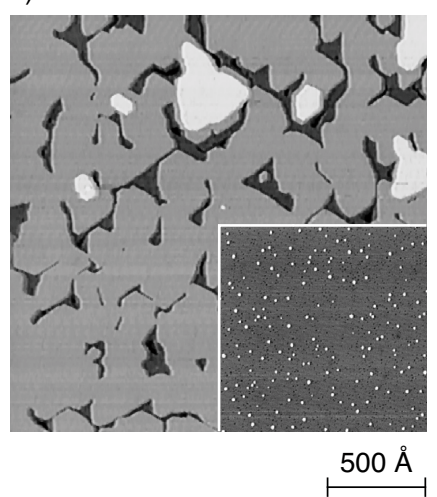

Figure 20.31 Growth manipulation for $\operatorname{Pt}(111)$ homoepitaxy ( $T_{\text {dep }}=400 \mathrm{~K}, \Theta=5 \mathrm{ML}, F=5 \times$ $\left.10^{-3} \mathrm{ML} \mathrm{s}^{-1}\right)$. (a) $3 \mathrm{D}$ growth induced by antisurfactant $\mathrm{CO}\left(p_{\mathrm{CO}}=5 \times 10^{-10} \mathrm{mbar}\right)$. (b) $2 \mathrm{D}$ growth induced by short sputter pulses applied at the beginning of each deposited monolayer ( $4 \mathrm{keV}$ $\mathrm{Ar}^{+}$ion pulses, each with a dose of $2 \times 10^{16}$ ions $\mathrm{m}^{-2}$ ). Inset: $\mathrm{Pt}(111)$ surface morphology after one sputter pulse. (Kindly provided by T. Michely and adapted from Ref. [286].) 
created after one sputter pulse on $\mathrm{Pt}(111)$ at $400 \mathrm{~K}$ [286]. The film morphology after deposition of $5 \mathrm{ML}$ at $400 \mathrm{~K}$ with periodic sputter pulses is almost perfectly flat (Figure 20.31b), while the slightly CO-contaminated system forms mounds (Figure 20.31a) very much as the clean system does, compare Figure 20.29a.

Periodic sputter pulses have also been applied with success to $\mathrm{Cu}(111)$ homoand $\mathrm{Ni} / \mathrm{Cu}(111)$ heteroepitaxy $[287,288]$. The drawback of growth manipulation by periodic ion bombardment is that subsurface vacancies created by the ion impact are not healed out for growth temperatures below 0.3 the melting temperature $T_{\mathrm{m}}$ [29] leading to porous films. An additional disadvantage in heteroepitaxy might be ion-impact-induced exchange of film with substrate atoms. However, the film morphology can be very well controlled this way, and the two kinds of possible point defects might not be disturbing for many applications.

\section{Further Reading}

This chapter is part of a textbook and therefore focused on the basic principles that are illustrated by a few examples taken for submonolayers mainly from the authors' past and in some cases also present own research and for multilayers from Thomas Michely who kindly provided many original figures. There are two more textbooks that we recommend. They are dedicated to graduate students, as well as suited to more experienced researchers as a first approach to the field. The one by John Venables [289] has a chapter dedicated to epitaxial growth where nucleation theory is outlined and also results on island densities and shapes from SEM are discussed. This book also contains valuable information on UHV technique and evaporators with many practical hints, and it describes the technique reflection high-energy electron diffraction (RHEED) that is commonly used to monitor the film morphology and the number of layers, which we did not discuss here. The second book is by Alberto Pimpinelli and Jacques Villain [290]. It focuses on crystal growth in the light of statistical physics and treats thermodynamic and kinetic concepts of growth, dissolution, ripening, and growth instabilities. It has a very useful collection of appendices with derivations and proofs where the more experienced reader can go very rapidly into depth.

Among the books dedicated to scientists that have already some experience in the field of epitaxial growth, we recommend the one of Thomas Michely and Joachim Krug [29]. It gives an excellent overview of our present understanding of submonolayer and multilayer growth in the kinetic growth regime. It focuses on homoepitaxy and therefore does not treat misfit, strain, and the related effects. For a more general, albeit less recent view on epitaxial growth, we recommend the edited book by David King and Phil Woodruff [291]. A second edited book that we recommend is by Zhenyu Zhang and Max Lagally [292]. It contains excellent chapters, for example, on the concept of two mobilities, surfactants, and coarsening. In addition, it gives an appreciation of the system-specific aspects for metal/metal, semiconductor/semiconductor, and metal/semiconductor epitaxy. Among the review articles, we recommend $[28,293]$ for submonolayer metal 
epitaxy, the book chapter [294] for their submonolayer and multilayer epitaxial growth, an early book chapter [295] and the review article [296] for semiconductors, and finally, [297] for SiC.

\section{Acknowledgments}

We gratefully acknowledge T. Michely for providing many figures for the multilayer part of this chapter and C.-L. Bandelier for preparation of all the figures.

\section{References}

1. Faist, J., Capasso, F., Sivco, D.L., Sirtori, C., Hutchinson, A.L., and Cho, A.Y. (1994) Quantum cascade laser. Science, 264, 553.

2. Faist, J., Capasso, F., Sirtori, C., Sivco, D., Hutchinson, A., and Cho, A. (1997) Laser action by tuning the oscillator strength. Nature, 387, 777.

3. Beck, M., Hofstetter, D., Aellen, T., Faist, J., Oesterle, U., Ilegems, M., Gini, E., and Melchior, H. (2002) Continuous wave operation of a midinfrared semiconductor laser at room temperature. Science, 295, 301.

4. Walther, C., Fischer, M., Scalari, G., Terazzi, R., Hoyler, N., and Faist, J. (2007) Quantum cascade lasers operating from 1.2 to $1.6 \mathrm{THz}$. Appl. Phys. Lett., 91, 131122.

5. Williams, B.S. (2007) Terahertz quantum-cascade lasers. Nat. Photon., 1, 517.

6. Tonouchi, M. (2007) Cutting-edge terahertz technology. Nat. Photon., 1, 97.

7. Grünberg, P., Schreiber, R., Pang, Y., Brodsky, M.B., and Sowers, H. (1986) Layered magnetic structures: evidence for antiferromagnetic coupling of $\mathrm{Fe}$ layers across $\mathrm{Cr}$ interlayers. Phys. Rev. Lett., 57, 2442.

8. Baibich, M.N., Broto, J.M., Fert, A., Nguyen Van Dau, F., Petroff, F., Etienne, P., Creuzet, G., Friederich, A., and Chazelas, J. (1988) Giant magnetoresistance of (001) Fe/(001) Cr magnetic superlattices. Phys. Rev. Lett., 61, 2472.
9. Mathon, J. and Umerski, A. (2001) Theory of tunneling magnetoresistance of an epitaxial $\mathrm{Fe} / \mathrm{MgO} / \mathrm{Fe}(001)$ junction. Phys. Rev. B, 63, 220403.

10. Zhang, X.G. and Butler, W.H. (2004) Large magnetoresistance in bcc $\mathrm{Co} / \mathrm{MgO} / \mathrm{Co}$ and $\mathrm{FeCo} / \mathrm{MgO} / \mathrm{FeCo}$ tunnel junctions. Phys. Rev. B, 70, 172407.

11. Miyazaki, T. and Tezuka, N. (1995) Giant magnetic tunneling effect in $\mathrm{Fe} / \mathrm{Al}_{2} \mathrm{O}_{3} / \mathrm{Fe}$ junction. J. Magn. Magn. Mater., 139, L231.

12. Moodera, J.S., Kinder, L.R., Wong, T.M., and Meservey, R. (1995) Large magnetoresistance at room temperature in ferromagnetic thin film tunnel junctions. Phys. Rev. Lett., 74, 3273.

13. Yuasa, S., Nagahama, T., Fukushima, A., Suzuki, Y., and Ando, K. (2004) Giant room-temperature magnetoresistance in single-crystal $\mathrm{Fe} / \mathrm{MgO} / \mathrm{Fe}$ magnetic tunnel junctions. Nat. Mater., 3, 868 .

14. Parkin, S.S.P., Kaiser, C., Panchula, A., Rice, P.M., Hughes, B., Samant, M., and Yang, S.H. (2004) Giant tunnelling magnetoresistance at room temperature with $\mathrm{MgO}(100)$ tunnel barriers. Nat. Mater., 3, 862.

15. Djayaprawira, D.D., Tsunekawa, K., Nagai, M., Maehara, H., Yamagata, S., Watanabe, N., Yuasa, S., Suzuki, Y., and Ando, K. (2005) $230 \%$ roomtemperature magnetoresistance in $\mathrm{CoFeB} / \mathrm{MgO} / \mathrm{CoFeB}$ magnetic tunnel junctions. Appl. Phys. Lett., 86, 092502.

16. Yuasa, S., Fukushima, A., Kubota, H., Suzuki, Y., and Ando, K. (2006) Giant 
tunneling magnetoresistance up to $410 \%$ at room temperature in fully epitaxial $\mathrm{Co} / \mathrm{MgO} / \mathrm{Co}$ magnetic tunnel junctions with bcc $\mathrm{Co}(001)$ electrodes. Appl. Phys. Lett., 89, 042505.

17. Wegrowe, J.E., Kelly, D., Jaccard, Y., Guittienne, P., and Ansermet, J.P. (1999) Current-induced magnetization reversal in magnetic nanowires. Europhys. Lett., 45, 626.

18. Myers, E.B., Ralph, D.C., Katine, J.A., Louie, R.N., and Buhrman, R.A. (1999) Current-induced switching of domains in magnetic multilayer devices. Science, 285, 867.

19. Wang, C., Cui, Y.T., Katine, J.A., Buhrman, R.A., and Ralph, D.C. (2011) Time-resolved measurement of spin-transfer-driven ferromagnetic resonance and spin torque in magnetic tunnel junctions. Nat. Phys., 7, 496.

20. Karpan, V.M., Giovannetti, G., Khomyakov, P.A., Talanana, M., Starikov, A.A., Zwierzycki, M., van den Brink, J., Brocks, G., and Kelly, P.J. (2007) Graphite and graphene as perfect spin filters. Phys. Rev. Lett., 99, 176602.

21. Gadzuk, J.W. (1996) Resonance-assisted hot electron femtochemistry at surfaces. Phys. Rev. Lett., 76, 4234.

22. Delong, A. and Kolarik, V. (1989) A 1:1 electron stepper. J. Vac. Sci. Technol., B, 7, 1422.

23. Poppeller, M., Cartier, E., and Tromp, R.M. (1998) Hot electron emission lithography. Appl. Phys. Lett., 73, 2835.

24. Eerenstein, W., Mathur, N.D., and Scott, J.F. (2006) Multiferroic and magnetoelectric materials. Nature, 442 , 759.

25. Eerenstein, W., Wiora, M., Prieto, J.L., Scott, J.F., and Mathur, N.D. (2007) Giant sharp and persistent converse magnetoelectric effects in multiferroic epitaxial heterostructures. Nat. Mater., 6, 348 .

26. Kaiser, N. and Pulker, H.K. (2003) Optical Interference Coatings, Springer Series in Optical Sciences, SpringerVerlag, Berlin, Heidelberg, New York.

27. Langer, J.S. (1980) Instabilities and pattern formation in crystal growth. Rev. Mod. Phys., 52, 1.
28. Brune, H. (1998) Microscopic view of epitaxial metal growth: nucleation and aggregation. Surf. Sci. Rep., 31, 121.

29. Michely, T. and Krug, J. (2004) Islands, Mounds, and Atoms, Vol. 42, Springer, Berlin.

30. Burton, W.K., Cabrera, N., and Frank, F.C. (1949) Role of dislocations in crystal growth. Nature, 163, 398.

31. Land, T.A., Michely, T., Behm, R.J., Hemminger, J.C., and Comsa, G. (1991) STM investigation of the adsorption and temperature dependent reactions of ethylene on $\operatorname{Pt}(111)$. Appl. Phys. A, 53, 414.

32. Vázquez de Parga, A.L., Calleja, F., Borca, B., Passeggi, M.C.G., Hinarejos, J.J., Guinea, F., and Miranda, R. (2008) Periodically rippled graphene: growth and spatially resolved electronic structure. Phys. Rev. Lett., 100, 056807.

33. Sutter, P.W., Flege, J.I., and Sutter, E.A. (2008) Epitaxial graphene on ruthenium. Nat. Mater., 7, 406.

34. Rusponi, S., Papagno, M., Moras, P., Vlaic, S., Etzkorn, M., Sheverdyaeva, P.M., Pacilé, D., Brune, H., and Carbone, C. (2010) Highly anisotropic dirac cones in epitaxial graphene modulated by an island superlattice. Phys. Rev. Lett., 105, 246803.

35. Papagno, M., Pacilé, D., Topwal, D., Moras, P., Sheverdyaeva, P.M., Natterer, F.D., Lehnert, A., Rusponi, S., Dubout, Q., Calleja, F., Frantzeskakis, E., Pons, S., Fujii, J., Vobornik, I., Grioni, M., Carbone, C., and Brune, H. (2012) Two distinct phases of bilayer graphene films on $\mathrm{Ru}(0001)$. ACS Nano, 6, 9299.

36. Martoccia, D., Willmott, P.R., Brugger, T., Björck, M., Günther, S., Schlepütz, C.M., Cervellino, A., Pauli, S.A., Patterson, B.D., Marchini, S., Wintterlin, J., Moritz, W., and Greber, T. (2008) Graphene on $\mathrm{Ru}(0001)$ : a $25 \times 25$ supercell. Phys. Rev. Lett., 101, 126102 .

37. Coraux, J., N'Diaye, A.T., Engler, M., Busse, C., Wall, D., Buckanie, N., Meyer zu Heringdorf, F.J., van Gastel, R., Poelsema, B., and Michely, T. (2009) Growth of graphene on $\operatorname{Ir}(111)$. New J. Phys., 11, 023006. 
38. Wang, B., Ma, X., Caffio, M., Schaub, R., and Li, W.X. (2011) Size-selective carbon nano-clusters as precursors to the growth of epitaxial graphene. Nano Lett., 11, 424

39. Wander, A., Van Hove, M.A., and Somorjai, G.A. (1991) Moleculeinduced displacive reconstruction in a substrate surface: ethylidyne adsorbed on $\mathrm{Rh}(111)$ studied by low-energyelectron diffraction. Phys. Rev. Lett., 67, 626.

40. Loginova, E., Bartelt, N.C., Feibelman, P.J., and McCarty, K.F. (2008) Evidence for graphene growth by C cluster attachment. New J. Phys., 10, 093026.

41. Loginova, E., Bartelt, M.C., Feibelman, P.J., and McCarty, K.F. (2009) Factors influencing graphene growth on metal surfaces. New J. Phys., 11, 063046.

42. Zangwill, A. and Vvedensky, D.D. (2011) Novel growth mechanism of epitaxial graphene on metals. Nano Lett., 11, 2092.

43. Natterer, F.D., Rusponi, S., Papagno, M., Carbone, C., and Brune, H. (2012) Optimizing long-range order, band gap, and group velocities for graphene on close-packed metal surfaces. J. Phys.: Condens. Matter, 24, 314203.

44. Arnoult, W.J. and McLellan, R.B. (1972) The solubility of carbon in rhodium ruthenium, iridium and rhenium. Scr. Metallur., 6, 1013.

45. José-Yacamán, M., Miki-Yoshida, M., Rendón, L., and Santiesteban, J.G. (1993) Catalytic growth of carbon microtubules with fullerene structure. Appl. Phys. Lett., 62, 657.

46. Bonard, J.M., Kind, H., Stöckli, T., and Nielsson, L.O. (2001) Field emission from carbon nanotubes: the first five years. Sol. State Electron., 45, 893.

47. Oshima, C. and Nagashima, A. (1997) Ultra-thin epitaxial films of graphite and hexagonal boron nitride on solid surfaces. J. Phys.: Condens. Matter, 9, 1.

48. Aleskovskii, V.B. (1974) Chemistry and technology of solids. J. Appl. Chem. USSR, 47, 2207.

49. Suntola, T. and Antson, J. (1977) Method for producing compound thin film. US Patent \#4,058,430, November 15.
50. Puurunen, R.L. (2005) Surface chemistry of atomic layer deposition: a case study for the trimethylaluminum/water process. J. Appl. Phys., 97, 121301.

51. Ritala, M. and Leskelä, M. (1999) Atomic layer epitaxy - a valuable tool for nanotechnology? Nanotechnology, 10, 19.

52. Leskelä, M. and Ritala, M. (2002) Atomic layer deposition (ALD): from precursors to thin film structures. Thin Solid Films, 409, 138.

53. Profijt, H.B., Potts, S.E., van de Sanden, M.C.M., and Kessels, W.M.M. (2011) Plasma-assisted atomic layer deposition: basics, opportunities, and challenges. J. Vac. Sci. Technol., A, 29, 050801.

54. Allongue, P. and Maroun, F. (2010) Electrodeposited magnetic layers in the ultrathin limit. MRS Bull., 35, 761.

55. Allongue, P. and Maroun, F. (2006) Metal electrodeposition on single crystal metal surfaces mechanisms, structure, and applications. Curr. Opin. Solid State Mater. Sci., 10, 173.

56. Allongue, P., Maroun, F., Jurca, H.F., Tournerie, N., Savidand, G., and Cortès, R. (2009) Magnetism of electrodeposited ultrathin layers: challenges and opportunities. Surf. Sci., 603, 1831.

57. Kolb, D.M., Przasnyski, M., and Gerischer, H. (1974) Underpotential deposition of metals and work function differences. J. Electroanal. Chem., 54, 25.

58. Budevski, E., Staikov, G., and Lorenz, W.J. (1996) Electrochemical Phase Formation and Growth - An Introduction to the Initial Stages of Metal Depositon, Wiley-VCH Verlag GmbH, Weinheim.

59. Möller, F.A., Magnussen, O.M., and Behm, R.J. (1996) Overpotentialcontrolled nucleation of $\mathrm{Ni}$ island arrays on reconstructed $\mathrm{Au}(111)$ electrode surfaces. Phys. Rev. Lett., 77, 5249.

60. Stuhlmann, C., Park, Z., Bach, C., and Wandelt, K. (1998) An in-situ and ex-situ study of Cd under-potential deposition on $\mathrm{Cu}(111)$. Electrochim. Acta, 44, 993. 
61. Magnussen, O.M., Polewska, W., Zitzler, L., and Behm, R.J. (2002) In situ video-STM studies of dynamic processes at electrochemical interfaces. Faraday Discuss., 121, 43.

62. Hommrich, J., Hümann, S., and Wandelt, K. (2002) Cadmium underpotential deposition on $\mathrm{Cu}(111)$ - in situ scanning tunneling microscopy. Faraday Discuss., 121, 129.

63. Stickney, J.L. (2002) Electrochemical deposition of II-VI semiconductors, in Advances in Electrochemical Sciences and Engineering, Vol. 7 (eds C. Alkire, D.M. Kolb, J. Lipkowski, and P.N. Ross), Wiley-VCH Verlag GmbH, Weinheim, p. 1.

64. Lincot, D. (2005) Electrodeposition of semiconductors. Thin Solid Films, 487, 40.

65. Kothari, H.M., Kulp, E.A., Limmer, S.J., Poizot, P., Bohannan, E.W., and Switzer, J.A. (2006) Electrochemical deposition and characterization of $\mathrm{Fe}_{3} \mathrm{O}_{4}$ films produced by the reduction of $\mathrm{Fe}(\mathrm{III})$-triethanolamine. J. Mater. Res., 21, 293.

66. Frank, F.C. and van der Merwe, J.H. (1949) One-dimensional dislocations. II. Misfitting monolayers and oriented overgrowth. Proc. R. Soc. London, Ser. A, 198, 216.

67. Volmer, M. and Weber, A. (1926) Keimbildung in übersättigten Gebilden. Z. Phys. Chem., 119, 277.

68. Stranski, I.N. and Krastanov, L. (1938) Zur Theorie der orientierten Ausscheidung von Ionenkristallen aufeinander. Sitzungsber. Akad. Wiss. Wien, Math.naturwiss. Kl. IIb, 146, 797.

69. Bauer, E. (1958) Phänomenologische Theorie der Kristallabscheidung an Oberflächen. I. Z. Kristallogr., 110, 372.

70. Zangwill, A. (1988) Physics at Surfaces, Cambridge University Press, New York.

71. Röder, H., Bromann, K., Brune, H., and Kern, K. (1997) Strain mediated two-dimensional growth kinetics in metal heteroepitaxy: Ag/Pt(111). Surf. Sci., 376, 13.

72. Bauer, E. and Van der Merwe, J.H. (1986) Structure and growth of crystalline superlattices: from monolayer to superlattice. Phys. Rev. B, 33, 3657.
73. Vitos, L., Ruban, A.V., Skriver, H.L., and Kollár, J. (1998) The surface energy of metals. Surf. Sci., 411, 186.

74. Abrahamson, J. (1973) The surface energies of graphite. Carbon, 11, 337.

75. Young, T. (1805) An essay on the cohesion of fluids. Philos. Trans. R. Soc. London, 95, 65.

76. Grabow, M.H. and Gilmer, G.H. (1988) Thin film growth modes, wetting and cluster nucleation. Surf. Sci., 194, 333.

77. Jesson, D.E., Chen, K.M., and Pennycook, S.J. (1996) Kinetic pathways to strain relaxation in the $\mathrm{Si}-\mathrm{Ge}$ system. Mater. Res. Soc. Bull., 21, 31.

78. Mo, Y.W., Savage, D.E., Swartzentruber, B.S., and Lagally, M.G. (1990) Kinetic pathway in stranskikrastanov growth of Ge on $\mathrm{Si}(001)$. Phys. Rev. Lett., 65, 1020.

79. Straub, M., Vollmer, B., and Kirschner, J. (1996) Surface magnetism of ultrathin $\gamma$-Fe films investigated by nonlinear magneto-optical Kerr effect. Phys. Rev. Lett., 77, 743.

80. Biedermann, A., Schmid, M., and Varga, P. (2001) Nucleation of bcc iron in ultrathin fcc films. Phys. Rev. Lett., 86, 464.

81. Rupp, W., Biedermann, A., Kamenik, B., Ritter, R., Klein, C., Platzgummer, E., Schmid, M., and Varga, P. (2008) Ion-beam induced fcc-bcc transition in ultrathin Fe films for ferromagnetic patterning. Appl. Phys. Lett., 93, 063102.

82. Brune, H. and Kern, K. (1997) Heteroepitaxial metal growth: the effects of strain, in Growth and Properties of Ultrathin Epitaxial Layers, The Chemical Physics of Solid Surfaces and Heterogeneous Catalysis, Vol. 8, (eds D.A. King and D.P. Woodruff), Elsevier Science, Amsterdam, p. 149.

83. LeGoues, F.K. (1996) The effect of strain on the formation of dislocations at the SiGe/Si interface. Mater. Res. Soc. Bull., 21, 21.

84. Cullis, A.G. (1996) Strain-induced modulations in the surface morphology of heteoepitaxial layers. Mater. Res. Soc. Bull., 21, 21.

85. Falub, C.V., von Känel, H., Isa, F., Bergamaschini, R., Marzegalli, A., Chrastina, D., Isella, G., Müller, E., 
Niedermann, P., and Miglio, L. (2012) Scaling hetero-epitaxy from layers to three-dimensional crystals. Science, 335, 1330.

86. Hitzke, A., Hugenschmidt, M.B., and Behm, R.J. (1997) Low temperature Ni atom adsorption on the $\mathrm{Au}(110)-(1 \times 2)$ surface. Surf. Sci., 389, 8 .

87. Günther, S., Hitzke, A., and Behm, R.J. (1997) Low adatom mobility on the (1x2)-missing-row reconstructed $\mathrm{Au}(110)$ surface. Surf. Rev. Lett., 4, 1103.

88. Brune, H., Bales, G.S., Boragno, C., Jacobsen, J., and Kern, K. (1999) Measuring surface diffusion from nucleation island densities. Phys. Rev. $B, 60,5991$

89. Vandoni, G., Félix, C., Monot, R., Buttet, J., and Harbich, W. (1994) Neighbour driven mobility of silver adatoms on $\operatorname{Pd}(100)$ measured by thermal helium scattering. Surf. Sci., 320, L63.

90. Wang, S.C. and Ehrlich, G. (1993) Adatom motion to lattice steps: a direct view. Phys. Rev. Lett., 70, 41.

91. Wang, S.C. and Ehrlich, G. (1993) Atom condensation at lattice steps and clusters. Phys. Rev. Lett., 71, 4174.

92. Kellogg, G.L. (1996) Experimental observation of ballistic atom exchange on metal surfaces. Phys. Rev. Lett., 76, 98 .

93. Voter, A.F. and Doll, J.D. (1984) Tranition state theory description of surface self-diffusion: comparison with classical trajectory theory. J. Chem. Phys., 80, 5832.

94. Gomer, R. (1990) Diffusion of adsorbates on metal surfaces. Rep. Prog. Phys., 53, 917.

95. Ehrlich, G. and Watanabe, F. (1991) Atomic interactions on crystals: a review of quantitative experiments. Langmuir, 7, 2555.

96. Watanabe, F. and Ehrlich, G. (1992) Direct observation of interactions between identical adatoms: Ir-Ir and Re-Re on W(110). J. Chem. Phys., 96, 3191.

97. Witten, T.A. and Sander, L.M. (1981) Diffusion limited aggregation, a kinetic critical phenomenon. Phys. Rev. Lett., 47, 1400 .

98. Meakin, P. (1988) The growth of fractal aggregates and their fractal measures, in Phase Tansitions and Critical Phenomena, Vol. 12 (eds C. Domb and J.L. Lebowitz), Academic Press, New York, p. 335.

99. Barabási, A.L. and Stanley, H.E. (1995) Fractal Concepts in Surface Growth, Cambridge University Press, New York.

100. Ehrlich, G. and Hudda, F.G. (1966) Atomic view of surface self-diffusion: tungsten on tungsten. J. Chem. Phys., 44, 1039.

101. Schwoebel, R.L. and Shipsey, E.J. (1966) Step motion on crystal surfaces. J. Appl. Phys., 37, 3682.

102. Stumpf, R. and Scheffler, M. (1994) Theory of self-diffusion at and growth of $\mathrm{Al}(111)$. Phys. Rev. Lett., 72, 254.

103. Yu, B.D. and Scheffler, M. (1996) Anisotropy of growth of the closepacked surfaces of silver. Phys. Rev. Lett., 77, 1095.

104. Feibelman, P.J. (1998) Interlayer selfdiffusion on stepped Pt(111). Phys. Rev. Lett., 81, 168.

105. Ehrlich, G. (1995) Atomic events at lattice steps and clusters: a direct view of crystal growth processes. Surf. Sci., 331-333, 865.

106. Meyer, J.A., Vrijmoeth, J., van der Vegt, H.A., Vlieg, E., and Behm, R.J. (1995) The importance of the additional step edge barrier in determining film morphology during epitaxial growth. Phys. Rev. B, 51, 14790.

107. Smilauer, P. and Harris, S. (1995) Determination of step-edge barriers to interlayer transport from surface morphology during the initial stages of homoepitaxial growth. Phys. Rev. B, 51, 14798.

108. Bromann, K., Brune, H., Röder, H., and Kern, K. (1995) Interlayer mass transport in homo- and heteroepitaxial metal growth. Phys. Rev. Lett., 75, 677.

109. Rottler, J. and Maass, P. (1999) Second layer nucleation in thin film growth. Phys. Rev. Lett., 83, 3490.

110. Heinrichs, S., Rottler, J., and Maass, P. (2000) Nucleation on top of islands in epitaxial growth. Phys. Rev. B, 62, 8338. 
111. Krug, J., Politi, P., and Michely, T. (2000) Island nucleation in the presence of step-edge barriers: theory and applications. Phys. Rev. B, 61, 14037.

112. Morgenstern, K., Rosenfeld, G., Lægsgaard, E., Besenbacher, F., and Comsa, G. (1998) Measurement of energies controlling ripening and annealing on metal surfaces. Phys. Rev. Lett., 80, 556.

113. Markov, I. (1997) Surface energetics from the transition from step-flow growth to two-dimensional nucleation in metal homoepitaxy. Phys. Rev. B, 56 , 12544.

114. Roos, K.R., Bhutani, R., and Tringides, M.C. (1997) Interlayer mass transport in a low coverage low island density regime. Surf. Sci., 384, 62.

115. Zhang, C.M., Bartelt, M.C., Wen, J.M., Jenks, C.J., Evans, J.W., and Thiel, P.A. (1997) The initial stages of $\mathrm{Ag} / \mathrm{Ag}(100)$ homoepitaxy: scanning tunneling microscopy experiments and montecarlo simulations. J. Cryst. Growth, 174, 851.

116. Alvarez, J., Lundgren, E., Torrelles, X., and Ferrer, S. (1998) Determination of scaling exponents in $\mathrm{Ag}(100)$ homoepitaxy with $\mathrm{x}$-ray diffractions profiles. Phys. Rev. B, 57, 6325.

117. Venables, J.A. (1973) Rate equation approaches to thin film nucleation kinetics. Philos. Mag., 17, 697.

118. Venables, J.A. (1987) Nucleation calculations in a pair-binding model. Phys. Rev. B, 36, 4153.

119. Liu, S., Bönig, L., and Metiu, H. (1995) Effect of small-cluster mobility and dissociation on the island density in epitaxial growth. Phys. Rev. B, 52, 2907.

120. Bartelt, M.C., Günther, S., Kopatzki, E., Behm, R.J., and Evans, J.W. (1996) Island-size distributions in submonolayer epitaxial growth: influence of the mobility of small clusters. Phys. Rev. B, 53, 4099.

121. Brune, H., Röder, H., Boragno, C., and Kern, K. (1994) Microscopic view of nucleation on surfaces. Phys. Rev. Lett., 73, 1955.

122. Venables, J.A., Spiller, G.D.T., and Hanbücken, M. (1984) Nucleation and growth of thin films. Rep. Prog. Phys., 47, 399.

123. Mo, Y.W., Kleiner, J., Webb, M.B., and Lagally, M.G. (1991) Activation energy for surface diffusion of $\mathrm{Si}$ on $\mathrm{Si}(001)$ : a scanning-tunneling-microscopy study. Phys. Rev. Lett., 66, 1998.

124. Stroscio, J.A., Pierce, D.T., and Dragoset, R.A. (1993) Homoepitaxial growth of iron and a real space view of reflection-high-energy-electron diffraction. Phys. Rev. Lett., 70, 3615.

125. Bott, M., Hohage, M., Morgenstern, M., Michely, T., and Comsa, G. (1996) New approach for determination of diffusion parameters of adatoms. Phys. Rev. Lett., 76, 1304.

126. Müller, B., Fischer, B., Nedelmann, L., Brune, H., and Kern, K. (1996) Initial stages of $\mathrm{Cu}$ epitaxy on $\mathrm{Ni}(100)$ : postnucleation and a well defined transition in critical island size. Phys. Rev. B, 54, 17858.

127. Bales, G.S. and Chrzan, D.C. (1994) Dynamics of irreversible island growth during submonolayer epitaxy. Phys. Rev. B, 50, 6057.

128. Brune, H., Bromann, K., Röder, H., Kern, K., Jacobsen, J., Stoltze, P., Jacobsen, K., and Nørskov, J. (1995) Effect of strain on surface diffusion and nucleation. Phys. Rev. B, 52, R14380.

129. Ratsch, C. and Scheffler, M. (1998) Density-functional theory calculations of hopping rates of surface diffusion. Phys. Rev. B, 58, 13163.

130. Barth, J.V., Brune, H., Fischer, B. Weckesser, J., and Kern, K. (2000) Dynamics of surface migration in the weak corrugation regime. Phys. Rev. Lett., 84, 1732.

131. Michely, T., Langenkamp, W., Hansen, H., and Busse, C. (2001) Comment on "Dynamics of surface migration in the weak corrugation regime". Phys. Rev. Lett., 86, 2695.

132. Busse, C., Langenkamp, W., Polop, C., Petersen, A., Hansen, H., Linke, U., Feibelman, P.J., and Michely, T. (2003) Dimer binding energies on $\mathrm{fcc}(111)$ metal surfaces. Surf. Sci., 539, L560.

133. Polop, C., Hansen, H., Langenkamp, W., Zhong, Z., Busse, C., Linke, U., 
Kotrla, M., Feibelman, P.J., and Michely, T. (2005) Oscillatory interaction between $\mathrm{O}$ impurities and al adatoms on $\mathrm{Al}(111)$ and its effect on nucleation and growth. Surf. Sci., 575, 89.

134. Fischer, B., Brune, H., Fricke, A., Barth, J.V., and Kern, K. (1999) Nucleation kinetics on inhomogeneous substrates: $\mathrm{Al} / \mathrm{Au}(111)$. Phys. Rev. Lett. 82, 1732.

135. Bogicevic, A., Ovesson, S., Hyldgaard, P., Lundqvist, B.I., Brune, H., and Jennison, D.R. (2000) Nature, strength, and consequences of indirect adsorbate interactions on metals. Phys. Rev. Lett., 85, 1910.

136. Venables, J.A. and Brune, H. (2002) Capture numbers in the presence of repulsive adsorbate interactions. Phys. Rev. B, 66, 195404.

137. Bartelt, M.C. and Evans, J.W. (1992) Scaling analysis of diffusion-mediated island growth in surface adsorption processes. Phys. Rev. B, 46, 12675.

138. Tang, L.H. (1993) Island formation in submonolayer epitaxy. J. Phys. I, 3, 935.

139. Ratsch, C., Zangwill, A., and Smilauer, P. (1994) Scaling of heteroepitaxial island sizes. Surf. Sci., 314, L937.

140. Stranick, S.J., Kamna, M.M., and Weiss, P.S. (1994) Atomic-scale dynamics of a two-dimensional gas-solid interface. Science, 266, 99.

141. Amar, J.G. and Family, F. (1995) Critical cluster size: Island morphology and size distribution in submonolayer epitaxial growth. Phys. Rev. Lett., 74, 2066.

142. Ovesson, S. (2002) Mean-field nucleation theory with nonlocal interactions. Phys. Rev. Lett., 88, 116102.

143. Fichthorn, K.A., Merrick, M.L., and Scheffler, M. (2002) A kinetic Monte Carlo investigation of island nucleation and growth in thin-film epitaxy in the presence of substrate-mediated interactions. Appl. Phys. A, 75, 17.

144. Repp, J., Moresco, F., Meyer, G., Rieder, K.H., Hyldgaard, P., and Persson, M. (2000) Substrate mediated long-range oscillatory interaction between adatoms: $\mathrm{Cu} / \mathrm{Cu}(111)$. Phys. Rev. Lett., 85, 2981.
145. Knorr, N., Brune, H., Epple, M., Hirstein, A., Schneider, A.M., and Kern, K. (2002) Long-range adsorbate interactions mediated by a two-dimensional electrons gas. Phys. Rev. B, 65, 115420.

146. Friedel, J. (1958) Metallic alloys. Nuovo Cimento Suppl., 7, 287.

147. Lau, K.H. and Kohn, W. (1978) Indirect long-range oscillatory interaction between adsorbed atoms. Surf. Sci., 75, 69.

148. Einstein, T.L. (1978) Comment on "oscillatory indirect interaction between adsorbed atoms" by K. H. Lau and W. Kohn. Surf. Sci., 75, 161.

149. Einstein, T.L. (1996) Interactions between adsorbed particles, in Handbook of Surface Science, Vol. 1 (ed. W.N. Unertl), Elsevier Science B. V., New York, p. 578.

150. Lau, K.H. and Kohn, W. (1977) Elastic interaction of two atoms adsorbed on a solid surface. Surf. Sci., 65, 607.

151. Wahlström, E., Ekvall, I., Olin, H., and Walldén, L. (1998) Long-range interaction between adatoms at the $\mathrm{Cu}(111)$ surface imaged by scanning tunelling microscopy. Appl. Phys. A, 66, S1107.

152. Matsumoto, Y. and Tanaka, K. (1998) Growth of nanosize Ni thin films on a modified $\mathrm{c}(2 \times 2)-\mathrm{N} \mathrm{Cu}(100)$ surface. Jpn. J. Appl. Phys., 37, L154.

153. Brune, H., Wintterlin, J., Ertl, G., and Behm, R.J. (1990) Direct imaging of adsorption sites and local electronic bonding effects on a metal surface: C/Al(111). Europhys. Lett., 13, 123.

154. Crommie, M.F., Lutz, C.P., and Eigler, D.M. (1993) Imaging standing waves in a two-dimensional electron gas. Nature, $363,524$.

155. Hasegawa, Y. and Avouris, Ph. (1993) Direct observation of standing wave formation of surface steps using scanning tunneling microscopy. Phys. Rev. Lett., 71, 1071.

156. Jeandupeux, O., Bürgi, L., Hirstein, A., Brune, H., and Kern, K. (1999) Thermal damping of quantum interference patterns of surface-state electrons. Phys. Rev. B, 59, 15926. 
157. Ashcroft, N.W. and Mermin, N.D. (1987) Solid State Physics, Saunders College, Philadelphia, PA.

158. Tsong, T.T. (1988) Experimental studies of the behavior of single adsorbed atoms on solid surfaces. Rep. Prog. Phys., 51, 759.

159. Trost, J., Zambelli, T., Wintterlin, J. and Ertl, G. (1996) Adsorbate-adsorbate interactions from statistical analysis of STM images: N/Ru(0001). Phys. Rev. B 54, 17850.

160. Hyldgaard, P. and Persson, M. (2000) Long-ranged adsorbate-adsorbate interactions mediated by a surface state band. J. Phys.: Condens. Matter, 12, L13.

161. Ovesson, S., Bogicevic, A., Wahnström, G., and Lundqvist, B.I. (2001) Neglected adsorbate interactions behind diffusion prefactor anomalies on metals. Phys. Rev. B, 64, 125423.

162. Stepanyuk, V.S., Baranov, A.N., Tsivlin, D.V., Hergert, W., Bruno, P., Knorr N., Schneider, M.A., and Kern, K. (2003) Quantum interference and long range adsorbate-adsorbate interactions. Phys. Rev. B, 68, 205410.

163. Silly, F., Pivetta, M., Ternes, M., Patthey, F., Pelz, J.P., and Schneider, W.D. (2004) Creation of an atomic superlattice by immersing metallic adatoms in a two-dimensional electron sea. Phys. Rev. Lett., 92, 016101.

164. Ding, H.F., Stepanyuk, V.S., Ignatiev, P.A., Negulyaev, N.N., Niebergall, L., Wasniowska, M., Gao, C.L., Bruno, P. and Kirschner, J. (2007) Self-organized long-period adatom strings on stepped metal surfaces: scanning tunneling microscopy, ab initio calculations, and kinetic monte carlo simulations. Phys. Rev. B, 76, 033409.

165. Pennec, Y., Auwärter, W., Schiffrin, A., Weber-Bargioni, A., Riemann, A. and Barth, J.V. (2007) Supramolecular gratings for tuneable confinement of electrons on metal surfaces. Nat. Nanotechnol., 2, 99.

166. Negulyaev, N.N., Stepanyuk, V.S. Hergert, W., Bruno, P., and Kirschner, J. (2008) Atomic-scale self-organization of Fe nanostripes on stepped $\mathrm{Cu}(111)$ surfaces: Molecular dynamics and
References

kinetic Monte Carlo simulations. Phys. Rev. B, 77, 085430.

167. Schiffrin, A., Reichert, J., Auwärter, W. Jahnz, G., Pennec, Y., Weber-Bargioni, A., Stepanyuk, V.S., Niebergall, L., Bruno, P., and Barth, J.V. (2008) Self-aligning atomic strings in surfacesupported biomolecular gratings. Phys. Rev. B, 78, 035424

168. Cheng, Z., Wyrick, J., Luo, M., Sun, D., Kim, D., Zhu, Y., Lu, W., Kim, K., Einstein, T.L., and Bartels, L. (2010) Adsorbates in a box: titration of substrate electronic states. Phys. Rev. Lett., 105, 066104

169. Pivetta, M., Pacchioni, G.E., Schlickum, U., Barth, J.V., and Brune, H. (2013) Formation of Fe cluster superlattice in a metal-organic quantum-box network. Phys. Rev. Lett., 110, 086102.

170. Brune, H., Giovannini, M., Bromann, K., and Kern, K. (1998) Self-organized growth of nanostructure arrays on strain-relief patterns. Nature, 394, 451.

171. Abbet, S., Riedo, E., Brune, H., Heiz, U., Ferrari, A.M., Giordano, L., and Pacchioni, G. (2001) Identification of defect sites on $\mathrm{MgO}(100)$ thin films by decoration with $\mathrm{Pd}$ atoms and studying $\mathrm{CO}$ adsorption properties. J. Am. Chem. Soc., 123, 6172.

172. Bogicevic, A. and Jennison, D.R. (2002) Effect of oxide vacancies on metal island nucleation. Surf. Sci., 515, L481.

173. Henry, C.R. (1998) Surface studies of supported model catalysts. Surf. Sci. Rep., 31, 231.

174. Haas, G., Menck, A., Brune, H., Barth, J.V., Venables, J.A., and Kern, K. (2000) Nucleation and growth of supported clusters at defect sites: $\mathrm{Pd} / \mathrm{MgO}(100)$. Phys. Rev. B, 61, 11105.

175. Ferrari, A.M. and Pacchioni, G. (1996 Metal deposition on oxide surfaces: a quantum-chemical study of the interaction of $\mathrm{Rb}, \mathrm{Pd}$, and $\mathrm{Ag}$ atoms with the surface vacancies of MgO. J. Phys. Chem., 100, 9032.

176. Sterrer, M., Risse, T., Pozzoni, U.M., Giordano, L., Heyde, M., Rust, H.P., Pacchioni, G., and Freund, H.J. (2007) Control of the charge state of metal atoms on thin MgO films. Phys. Rev. Lett., 98, 096107. 
177. Heiz, U., Abbet, S., Sanchez, A., and Schneider, W.D. (1999) Catalytic oxidation of carbon monoxide on monodispersed platinum clusters: each atom counts. J. Am. Chem. Soc., 121, 3214.

178. Ricci, D., Bongiorno, A., Paccioni, G., and Landman, U. (2006) Bonding trends and dimensionality crossover of gold nanoclusters on metal-supported MgO thin films. Phys. Rev. Lett., 97 36106.

179. Zhang, C., Yoon, B., and Landman, U. (2007) Predicted oxidation of CO catalyzed by $\mathrm{Au}$ nanoclusters on a thin defect-free $\mathrm{MgO}$ film supported on a Mo(100) surface. J. Am. Chem. Soc., 129, 2228.

180. Simic-Milosevic, V., Heyde, M., Nilius, N., König, T., Rust, H.P., Sterrer, M., Risse, T., Freund, H.J., Giordano, L. and Pacchioni, G. (2008) Au dimers on thin $\mathrm{MgO}(001)$ films: flat and charged or upright and neutral?. J. Am. Chem. Soc., 130, 7814

181. Sljivancanin, Z., Brune, H., and Pasquarello, A. (2009) Nitrogen fixation at passivated Fe nanoclusters supported by an oxide surface: identification of viable reaction routes using density functional calculations. Phys. Rev. B, 80, 075407.

182. N'Diaye, A.T., Coraux, J., Plasa, T.N., Busse, C., and Michely, T. (2008) Structure of epitaxial graphene on $\operatorname{Ir}(111)$ New J. Phys., 10, 043033.

183. Degen, S., Becker, C., and Wandelt, K. (2004) Thin alumina films on $\mathrm{Ni}_{3} \mathrm{Al}(111)$ : a template for nanostructured Pd cluster growth. Faraday Discuss., 125, 343.

184. Brune, H. (2006) Giant spinpolarization and magnetic anisotropy of nanostructures at surfaces. e-J. Surf. Sci. Nanotechnol., 4, 478.

185. N'Diaye, A.T., Bleikamp, S., Feibelman, P.J., and Michely, T. (2006) Twodimensional Ir cluster lattice on a graphene moiré on Ir(111). Phys. Rev. Lett., 97, 215501.

186. Balog, R., Jørgensen, B., Nilsson, L., Andersen, M., Rienks, E., Bianchi, M., Fanetti, M., Lægsgaard, E. Baraldi, A., Lizzit, S., Sljivancanin,
Z., Besenbacher, F., Hammer, B. Pedersen, T.G., Hofmann, P., and Hornekær, L. (2010) Bandgap opening in graphene induced by patterned hydrogen adsorption. Nat. Mater., 9, 315.

187. Schmid, M., Kresse, G., Buchsbaum, A., Napetschnig, E., Gritschneder, S., Reichling, M., and Varga, P. (2007) Nanotemplate with holes: ultrathin alumina on $\mathrm{Ni}_{3} \mathrm{Al}(111)$. Phys. Rev. Lett. 99, 196104.

188. Repain, V., Baudot, G., Ellmer, H., and Rousset, S. (2002) Two-dimensional long-range-ordered growth of uniform cobalt nanostructures on a $\mathrm{Au}(111)$ vicinal template. Europhys. Lett., 58 730.

189. Barth, J.V., Brune, H., Ertl, G., and Behm, R.J. (1990) Scanning tunneling microscopy on the reconstructed $\mathrm{Au}(111)$ surface: atomic structure, longrange rotational domains, and surface defects. Phys. Rev. B, 42, 9307.

190. Weiss, N., Cren, T., Epple, M., Rusponi, S., Baudot, G., Rohart, S. Tejeda, A., Repain, V., Rousset, S., Ohresser, P., Scheurer, F., Bencok, P., and Brune, H. (2005) Uniform magnetic properties for an ultrahighdensity lattice of noninteracting $\mathrm{Co}$ nanostructures. Phys. Rev. Lett., 95, 157204.

191. Repain, V., Rohart, S., Girard, Y. Tejeda, A., and Rousset, S. (2006) Building uniform and long-range ordered nanostructures on a surface by nucleation on a point defect array. $J$. Phys.: Condens. Matter, 18, S17.

192. Rohart, S., Girard, Y., Nahas, Y., Repain, V., Rodary, G., Tejeda, A., and Rousset, S. (2008) Growth of iron on gold (788) vicinal surface: from nanodots to step flow. Surf. Sci., 602, 28.

193. Lehnert, A., Buluschek, P., Weiss, N. Giesecke, J., Treier, T., Rusponi, S., and Brune, H. (2009) High resolution in situ magneto-optic Kerr effect and scanning tunneling microscopy setup with all optical components in UHV. Rev. Sci. Instrum., 80, 023902.

194. Vicsek, T. (1989) Fractal Growth Phenomena, World Scientific, Singapore. 
195. Takayasu, H. (1990) Fractals in the physical sciences, Manchester University Press, Manchester, New York.

196. Furukawa, Y. (1997) Faszination der Schneekristalle - wie ihre bezaubernden Formen entstehen. Chem. Unserer Zeit, 31, 58.

197. Hwang, R.Q., Schröder, J., Günther, C., and Behm, R.J. (1991) Fractal growth of two-dimensional islands: Au on Ru(0001). Phys. Rev. Lett., 67, 3279.

198. Röder, H., Hahn, E., Brune, H., Bucher, J.P., and Kern, K. (1993) Building one- and two-dimensional nanostructures by diffusion-controlled aggregation at surfaces. Nature, 366, 141.

199. Brune, H., Romainczyk, C., Röder, H., and Kern, K. (1994) Mechanism of the transition from fractal to dendritic growth of surface aggregates. Nature, 369, 469.

200. Michely, T., Hohage, M., Bott, M., and Comsa, G. (1993) Inversion of growth speed anisotropy in two dimensions. Phys. Rev. Lett., 70, 3943.

201. Brune, H., Röder, H., Bromann, K., Kern, K., Jacobsen, J., Stoltze, P., Jacobsen, K., and Nørskov, J. (1996) Anisotropic corner diffusion as origin for dendritic growth on hexagonal substrates. Surf. Sci., 349, L115.

202. Couder, Y., Gérard, N., and Rabaud, M. (1986) Narrow fingering in the Saffman-Taylor instability. Phys. Rev. A, 34, 5175.

203. Pimpinelli, A., Villain, J., and Wolf, D.E. (1993) Fractal terraces in MBE growth. J. Phys. (Paris), 3, 447.

204. Bartelt, M.C. and Evans, J.W. (1994) Dendritic islands in metal-on-metal epitaxy I. Shape transtitions and diffusion at island edges. Surf. Sci., 314, L829.

205. Bales, G.S. and Chrzan, D.C. (1995) Transition from compact to fractal islands during submonolayer epitaxial growth. Phys. Rev. Lett., 74, 4879.

206. Röder, H., Bromann, K., Brune, H., and Kern, K. (1995) Diffusion-limited aggregation with active edge diffusion. Phys. Rev. Lett., 74, 3217.

207. Stoltze, P. (1994) Simulations of surface defects. J. Phys.: Condens. Matter, 6, 9495.
208. Ruggerone, P., Ratsch, C., and Scheffler, M. (1997) Density functional theory of epitaxial growth of metals, in Growth and Properties of Ultrathin Epitaxial Layers, The Chemical Physics of Solid Surfaces and Heterogeneous Catalysis, Vol. 8 (eds D.A. King and D.P. Woodruff), Elsevier Science, Amsterdam, p. 490.

209. Liu, C.L. (1994) Energetics of diffusion processes during nucleation and growth for the $\mathrm{Cu} / \mathrm{Cu}(100)$ system. Surf. Sci., 316, 294.

210. Chambliss, D.D. and Johnson, K.E. (1994) Nucleation with a critical cluster size of zero: submonolayer Fe inclusion in $\mathrm{Cu}(100)$. Phys. Rev. B, 50 5012.

211. Kopatzki, E., Günther, S., Nichtl-Pecher, W., and Behm, R.J. (1993) Homoepitaxial growth on $\mathrm{Ni}(100)$ and its modification by a preadsorbed oxygen adlayer. Surf. Sci. 284, 154.

212. Hahn, E., Kampshoff, E., Wälchli, N., and Kern, K. (1995) Strain driven fccbct phase transition of pseudomorphic $\mathrm{Cu}$ films on Pd(100). Phys. Rev. Lett., 74, 1803.

213. Wen, J.M., Evans, J.W., Bartelt, M.C., Burnett, J.W., and Thiel, P.A. (1996) Coarsening mechanisms in a metal film: from cluster diffusion to vacancy ripening. Phys. Rev. Lett., 76, 652.

214. Zhang, Z., Chen, X., and Lagally, M.G. (1994) Bonding-geometry dependence of fractal growth on metal surfaces. Phys. Rev. Lett., 73, 1829.

215. Müller, B., Nedelmann, L., Fischer, B., Brune, H., Barth, J.V., and Kern, K. (1998) Island shape transition in heteroepitaxial metal growth on square lattices. Phys. Rev. Lett., 80, 2642.

216. Hohage, M., Bott, M., Morgenstern, M., Zhang, Z., Michely, T., and Comsa, G. (1996) Atomic processes in low temperature Pt-dendrite growth on $\mathrm{Pt}(111)$. Phys. Rev. Lett., 76, 2366.

217. Buatier de Mongeot, F., Scherer, M., Gleich, B., Kopatzki, E., and Behm, R.J. (1998) CO adsorption and oxidation on bimetallic $\mathrm{Pt} / \mathrm{Ru}(0001)$ surfaces - a combined STM and TPD/TPR study. Surf. Sci., 411, 249. 
218. Nørskov, J.K., Jacobsen, K.W., Stoltze, P., and Hansen, L.B. (1993) Many-atom interactions on metals. Surf. Sci., 283, 277.

219. Feibelman, P. (1999) Self-diffusion along step bottoms on Pt(111). Phys. Rev. B, 60, 4972.

220. Günther, C., Günther, S., Kopatzki, E., Hwang, R.Q., Schröder, J., Vrijmoeth, J., and Behm, R.J. (1993) Microscopic aspects of thin metal film epitaxial growth on metallic substrates. Ber. Bunsenges. Phys. Chem., 97, 522.

221. Bromann, K., Brune, H., Giovannini, M., and Kern, K. (1997) Pseudomorphic growth induced by chemical adatom potential. Surf. Sci., 388, L1107.

222. Bromann, K., Félix, C., Brune, H., Harbich, W., Monot, R., Buttet, J. and Kern, K. (1996) Controlled deposition of size-selected Ag nanoclusters. Science, 274, 956.

223. Bogicevic, A., Liu, S., Jacobsen, J., Lundquist, B.I., and Metiu, H.I. (1998) Island migration caused by the motion of atoms at the border: the size and temperature dependence of the diffusion coefficient. Phys. Rev. B, 57, R9459.

224. Bogicevic, A., Strömquist, J., and Lundqvist, B.I. (1998) Low-symmetry diffusion barriers in homoepitaxial growth of Al(111). Phys. Rev. Lett., 81, 637.

225. Fichthorn, K.A. and Scheffler, M. (2000) Island nucleation in thin-film epitaxy: a first-principles investigation. Phys. Rev. Lett., 84, 5371.

226. Fichthorn, K.A., Merrick, M.L., and Scheffler, M. (2003) Nanostructures at surfaces from substrate-mediated interactions. Phys. Rev. B, 68, 041404.

227. Hwang, R.Q., Günther, C., Schröder, J., Günther, S., Kopatzki, E., and Behm, R.J. (1992) Nucleation and growth of thin metal films on clean and modified metal substrates studied by scanning tunneling microscopy. $J$. Vac. Sci. Technol., A, 10, 1970.

228. Vrijmoeth, J., Günther, C., Schröder, J., Hwang, R.Q., and Behm, R.J. (1993) Morphology and structure of ultrathin $\mathrm{Co}$ - and $\mathrm{Au}$-films grown on $\mathrm{Ru}(0001)$ substrates, in Magnetism and Structure in Systems of Reduced Dimension (ed. R.F.C. Farrow), Plenum Press, New York, p. 55.

229. Meier, F., von Bergmann, K., Ferriani, P., Wiebe, J., Bode, M., Hashimoto, K., Heinze, S., and Wiesendanger, R. (2006) Spin-dependent electronic and magnetic properties of Co nanostructures on $\mathrm{Pt}(111)$ studied by spin-resolved scanning tunneling spectroscopy. Phys. Rev. B, 74, 195411.

230. Voigtländer, B., Meyer, G., and Amer, N.M. (1991) Epitaxial growth of Fe on $\mathrm{Au}(111)$ : a scanning tunneling microscopy investigation. Surf. Sci., 255, L529.

231. Stroscio, J.A., Pierce, D.T., Dragoset, R.A., and First, P.N. (1992) Microscopic aspects of the initial growth of metastable fcc iron on $\mathrm{Au}(111)$. J. Vac. Sci. Technol., A, 10, 1981.

232. Kalff, M., Comsa, G., and Michely, T. (1998) How sensitive is epitaxial growth to adsorbates? Phys. Rev. Lett., 81, 1255.

233. Ovesson, S., Bogicevic, A., and Lundqvist, B.L. (1999) Origin of compact triagular islands in metal-on-metal growth. Phys. Rev. Lett., 83, 2608.

234. Michely, T. and Comsa, G. (1991) Temperature dependence of the sputtering morphology of Pt(111). Surf. Sci., 256, 217.

235. Arenhold, K., Surnev, S., Bonzel, H.P., and Wynblatt, P. (1999) Step energetics of $\mathrm{Pb}(111)$ vicinal surfaces from facet shape. Surf. Sci., 424, 271.

236. Nowicki, M., Bombis, C., Emundts, A., and Bonzel, H.P. (2003) Absolute step and kink formation energies of $\mathrm{Pb}$ derived from step roughening of two-dimensional islands and facets. Phys. Rev. B, 67, 075405.

237. Ostwald, W. (1900) On the assumed isomerism of red and yellow mercury oxide and the surface-tension of solid bodies. Z. Phys. Chem., 34, 495.

238. Theis, W., Bartelt, N.C., and Tromp, R.M. (1995) Chemical potential maps and spatial correlations in 2D-island ripening on $\mathrm{Si}(001)$. Phys. Rev. Lett., 75, 3328. 
239. Lifshitz, L.M. and Slyozov, V.V. (1961) The kinetics of precipitation from supersaturated solid solutions. J. Phys. Chem. Solids, 19, 35.

240. Wagner, C. (1961) Theorie der Alterung von Niederschlägen durch Umlösen (Ostwald Reifung). Z. Elektrochem., 65, 581.

241. Chakraverty, B.K. (1967) Grain size distribution in thin films: 1 . Conservative systems. J. Phys. Chem. Solids, 28, 2401.

242. Wynblatt, P. and Gjostein, N.A. (1975) Supported metal crystallites, in Progress in Solid State Chemistry, Vol. 9 (eds J.O. McCardin and G. Somorjai), Pergamon, Oxford, p. 21.

243. Morgenstern, K., Rosenfeld, G., and Comsa, G. (1996) Decay of twodimensional $\mathrm{Ag}$ islands on $\mathrm{Ag}(111)$. Phys. Rev. Lett., 76, 2113.

244. Hannon, J.B., Klünker, C., Giesen, M., Ibach, H., Bartelt, N.C., and Hamilton, J.C. (1997) Surface self-diffusion by vacancy motion: Island ripening on $\mathrm{Cu}(100)$. Phys. Rev. Lett., 79, 2506.

245. Pearson, C., Borovsky, B., Krueger, M., Curtis, R., and Ganz, E. (1995) Si(001) step dynamics. Phys. Rev. Lett., 74, 2710.

246. van Gastel, R., Somfai, E., van Saarloos, W., and Frenken, J.W.M. (2000) A giant atomic slide puzzle. Nature, 408, 665.

247. van Gastel, R., Somfai, E., von Albada, S.B., van Saarloos, W., and Frenken, J.W.M. (2001) Nothing moves a surface: vacancy mediated surface diffusion. Phys. Rev. Lett., 86, 1562.

248. Jena, P., Khanna, S.N., and Rao, B.K. (1992) Physics and Chemistry of Finite Systems: From Clusters to Crystals, Kluwer, Dordrecht.

249. Gambardella, P., Rusponi, S., Veronese, M., Dhesi, S.S., Grazioli, C., Dallmeyer, A., Cabria, I., Zeller, R., Dederichs, P.H., Kern, K., Carbone, C., and Brune, H. (2003) Giant magnetic anisotropy of single Co atoms and nanoparticles on Pt. Science, 300, 1130.

250. Brune, H. (2000) Growth of metal clusters at surfaces, in Metal Clusters at Surfaces, Springer Series in Cluster Physics (ed. K.H. Meiwes-Broer), Springer, Berlin, p. 67.
251. Blandin, P., Massobrio, C., and Ballone, P. (1994) Evidence for strain induced 2D roughening in Ag islands on Pt(111). Phys. Rev. Lett., 72, 3072.

252. Röder, H., Brune, H., and Kern, K. (1994) Roughening and fragmentation of strained Ag islands on Pt(111). Phys. Rev. Lett., 73, 2143.

253. Rusponi, S., Cren, T., Longwitz, S.R., Lehnert, A., Moulas, G., Buluschek, P., Goyhenex, C., Vargoz, E., Kern, K., and Brune, H. (2013) Ripening by cluster diffusion, inpreparation.

254. Buluschek, P. (2007) Submonolayer growth of cobalt on metallic and insulating surfaces studied by scanning tunneling microscopy and kinetic Monte-Carlo simulations. PhD thesis, Swiss Federal Institute of Technology of Lausanne.

255. Buluschek, P., Rusponi, S., Vargoz, E., El Ouali, M., Kern, K., and Brune, H. Nucleation in the presence of insertion - Co/Pt(111), inpreparation.

256. Ternes, M., Lutz, C.P., Hirjibehedin, C.F., Giessibl, F.J., and Heinrich, A.J. (2008) The force needed to move an atom on a surface. Science, 319, 1066.

257. Jorritsma, L.C., Bijnagte, M., Rosenfeld, G., and Poelsema, B. (1997) Growth anisotropy and pattern formation in metal epitaxy. Phys. Rev. Lett., 78, 911.

258. Seah, M.P. (1972) Quantitative auger electron spectroscopy and electron ranges. Surf. Sci., 32, 703.

259. Kalff, M., Smilauer, P., Comsa, G., and Michely, T. (1999) No coarsening in Pt(111) homoepitaxy. Surf. Sci., 426, L447.

260. Meinel, K., Klaua, M., and Bethge, H. (1988) The influence of the substrate microtopography on the growth of thin films studied for the Au-Ag system by TEM and AES. J. Cryst. Growth, 89, 447.

261. Luo, E.Z., Wollschläger, J., Wegner, F., and Henzler, M. (1995) SPA-LEED studies of growth of $\mathrm{Ag}$ on $\mathrm{Ag}(111)$ at low temperatures. Appl. Phys. A, 60, 19.

262. Rosenfeld, G., Lipkin, N.N., Wulfhekel, W., Kliewer, J., Morgenstern, K., Poelsema, B., and Comsa, G. (1995) New concepts for controlled homoepitaxy. Appl. Phys. A, 61, 455. 
263. Brune, H. (2001) Metals on metals, in Physics of Covered Solid Surfaces, Subvolume A, Part 1 of Landolt Börnstein New Series, Group III: Condensed Matter, Vol. III/42 (ed. H.P. Bonzel), Springer, Berlin, p. 217.

264. Kunkel, R., Poelsema, B., Verheij, L.K., and Comsa, G. (1990) Reentrant layerby-layer growth during molecular-beam epitaxy of metal-on-metal substrates. Phys. Rev. Lett., 65, 733.

265. Michely, T., Hohage, M., Esch, S., and Comsa, G. (1996) The effect of surface reconstruction on the growth mode in homoepitaxy. Surf. Sci., 349, L89.

266. Jacobsen, J., Jacobsen, K.W., and Stoltze, P. (1994) Nucleation of the Pt(111) reconstruction: a simulation study. Surf. Sci., 317, 8.

267. Rosenfeld, G., Poelsema, B., and Comsa, G. (1995) The concept of two mobilities in homoepitaxial growth. $J$. Cryst. Growth, 151, 230.

268. Rosenfeld, G., Comsa, G., and Poelsema, B. (1998) The concept of two mobilities for growth manipulation, in Morphological Organization in Epitaxial Growth and Remošal, Directions in Condensed Matter Physics, Vol. 14 (eds Z. Zhang and M.G. Lagally), World Scientific, Singapore, p. 349.

269. Copel, M., Reuter, M.C., Kaxiras, E. and Tromp, R.M. (1989) Surfactants in epitaxial growth. Phys. Rev. Lett., 63, 632

270. Kandel, D. and Kaxiras, E. (2000) The surfactant effect in semiconductor thinfilm growth. Solid State Phys.: Adv. Res. Appl., 54, 219.

271. Vrijmoeth, J., van der Vegt, H.A., Meyer, J.A., Vlieg, E., and Behm, R.J. (1994) Surfactant-induced layer-by-layer growth of $\mathrm{Ag}$ on $\mathrm{Ag}(111)$ : origins and side effects. Phys. Rev. Lett., 72, 3843.

272. van der Vegt, H.A., van Pinxteren, H.M., Lohmeier, M., Vlieg, E., and Thornton, J.M. (1992) Surfactantinduced layer-by-layer growth of Ag on Ag(111). Phys. Rev. Lett., 68, 3335.

273. Li, M., Chung, P.W., Cox, E., Jenks, C.J., Thiel, P.A., and Evans, J.W. (2008) Exploration of complex multilayer film growth morphologies: Stm analysis and predictive atomistic modeling for $\mathrm{Ag}$ on Ag(111). Phys. Rev. B, 77, 033402.

274. Oppo, S., Fiorentini, V., and Scheffler, M. (1993) Theory of adsorption and surfactant effect of Sb on $\mathrm{Ag}(111)$. Phys. Rev. Lett., 71, 2437.

275. Brune, H., Wintterlin, J., Trost, J., Ertl, G., Wiechers, J., and Behm, R.J. (1993) Interaction of oxygen with $\mathrm{Al}(111)$ stud ied by scanning tunneling microscopy. J. Chem. Phys., 99, 2128.

276. Trost, J., Brune, H., Wintterlin, J., Behm, R.J., and Ertl, G. (1998) The interaction of oxygen with $\mathrm{Al}(111)$ at high temperatures. J. Chem. Phys., 108 1740.

277. Poelsema, B., Kunkel, R., Nagel, N., Becker, A.F., Rosenfeld, G., Verheij, L.K., and Comsa, G. (1991) New phenomena in homoepitaxial growth of metals. Appl. Phys. A, 53, 369.

278. Wolter, H., Schmidt, M., and Wandelt, K. (1993) Surfactant induced layerby-layer growth of $\mathrm{Cu}$ on $\mathrm{Ru}(0001)$ as revealed by oscillatory work function changes. Surf. Sci., 298, 173.

279. Schmidt, M., Wolter, H., and Wandelt, K. (1994) Work-function oscillations during the surfactant induced layerby-layer growth of copper on oxygen precovered Ru(0001). Surf. Sci., 307309, 507.

280. Esch, S., Hohage, M., Michely, T., and Comsa, G. (1994) Origin of oxygen induced layer-by-layer growth in homoepitaxy on Pt(111). Phys. Rev. Lett., 72, 518.

281. Camarero, J., Ferrón, J., Cros, V., Gómez, L., Vázquez de Parga, A.L., Gallego, J.M., Prieto, J.E., de Miguel, J.J., and Miranda, R. (1998) Atomistic mechanism of surfactant-assisted epitaxial growth. Phys. Rev. Lett., 81, 850.

282. Baumberger, F., Tamai, A., Muntwiler, M., Greber, T., and Osterwalder, J. (2003) The electronic structure of a surfactant layer: $\mathrm{Pb} / \mathrm{Cu}(111)$. Surf. Sci., 532, 82.

283. Camarero, J., Graf, T., de Miguel, J.J., Miranda, R., Kuch, W., Zharnikov, M., Dittschar, A., Schneider, C.M., and Kirschner, J. (1996) Surfactantmediated modification of the magnetic 
properties of $\mathrm{Co} / \mathrm{Cu}(111)$ thin films and superlattices. Phys. Rev. Lett., 76, 4428.

284. Rosenfeld, G., Servaty, R., Teichert, C. Poelsema, B., and Comsa, G. (1993) Layer-by-layer growth of $\mathrm{Ag}$ on $\mathrm{Ag}(111)$ induced by enhanced nucleation: a model study for surfactant-mediated growth. Phys. Rev. Lett., 71, 895.

285. Michely, T. and Comsa, G. (1991) Generation and nucleation of adatoms during ion bombardement of $\mathrm{Pt}(111)$. Phys. Rev. B, 44, 8411.

286. Esch, S., Breemann, M., Morgenstern, M., Michely, T., and Comsa, G. (1996) Nucleation and morphology of homoepitaxial Pt(111)-films grown with ion beam assisted deposition. Surf. Sci., 365, 187.

287. Wulfhekel, W., Lipkin, N.N., Kliewer, J., Rosenfeld, G., Jorritsma, L.C., Poelsema, B., and Comsa, G. (1996) Conventional and manipulated growth of $\mathrm{Cu} / \mathrm{Cu}(111)$. Surf. Sci., 348, 227.

288. Wulfhekel, W., Beckmann, I., Lipkin, N.N., Rosenfeld, G., Poelsema, B., and Comsa, G. (1996) Manipulation of growth modes in heteroepitaxy: Ni/Cu(111). Appl. Phys. Lett., 69, 3492.

289. Venables, J.A. (2000) Introduction to Surface and Thin Film Processes, Cambridge University Press, Cambridge.

290. Pimpinelli, A. and Villain, J. (1998) Physics of Crystal Growth, Cambridge University Press, Cambridge.
291. King, D.A. and Woodruff, D.P. (1997) Growth and Properties of Ultrathin Epitaxial Layers, The Chemical Physics of Solid Surfaces, Vol. 8, Elsevier Science, Amsterdam.

292. Zhang, Z. and Lagally, M.G. (1998) Morphological Organization in Epitaxial Growth and Removal, Series in Direction in Condensed Matter Physics, Vol. 14, World Scientific, Singapore.

293. Evans, J.W., Thiel, P.A., and Bartelt, M.C. (2006) Morphological evolution during epitaxial thin film growth: formation of 2D islands and 3D mounds. Surf. Sci. Rep., 61, 1.

294. Bauer, E. (1984) Metals on metals, in Chemisorption Systems, The Chemical Physics of Solid Surfaces and Heterogeneous Catalysis, Part B, Vol. 3B (eds D.A. King and D.P. Woodruff), Elsevier Science, Amsterdam, p. 1.

295. Lagally, M.G., Mo, Y.W., Kariotis, R., Swartzentruber, B.S., and Webb, M.B. (1990) Microscopic aspects of the initial stages of epitaxial growth: a scanning tunneling microscopy study of $\mathrm{Si}$ on $\mathrm{Si}(001)$, in Kinetics of Ordering and Growth at Surfaces (ed. M.G. Lagally), Plenum Press, New York, p. 145.

296. Joyce, B.A. and Vvedensky, D.D. (2004) Self-organized growth on GaAs surfaces. Mater. Sci. Eng. R, 46, 127.

297. Masri, P. (2002) Silicon carbide and silicon carbide-based structures - the physics of epitaxy. Surf. Sci. Rep., 48, 1. 